University of Rhode Island

DigitalCommons@URI

Open Access Dissertations

2020

\title{
LARGE EDDY SIMULATION OF TURBULENT AIRFLOW OVER SURFACE WAVES AND AIR-SEA FLUXES
}

Nyla Tauqir Husain

University of Rhode Island, nyla.husain@gmail.com

Follow this and additional works at: https://digitalcommons.uri.edu/oa_diss

\section{Recommended Citation}

Husain, Nyla Tauqir, "LARGE EDDY SIMULATION OF TURBULENT AIRFLOW OVER SURFACE WAVES AND AIR-SEA FLUXES" (2020). Open Access Dissertations. Paper 1221.

https://digitalcommons.uri.edu/oa_diss/1221

This Dissertation is brought to you for free and open access by DigitalCommons@URI. It has been accepted for inclusion in Open Access Dissertations by an authorized administrator of DigitalCommons@URI. For more information, please contact digitalcommons-group@uri.edu. 
LARGE EDDY SIMULATION OF TURBULENT AIRFLOW OVER SURFACE WAVES AND AIR-SEA FLUXES

BY

NYLA TAUQIR HUSAIN

A DISSERTATION SUBMITTED IN PARTIAL FULFILLMENT OF THE REQUIREMENTS FOR THE DEGREE OF

DOCTOR OF PHILOSOPHY

IN

OCEANOGRAPHY

UNIVERSITY OF RHODE ISLAND

2020 


\title{
DOCTOR OF PHILOSOPHY DISSERTATION \\ $\mathrm{OF}$
}

NYLA TAUQIR HUSAIN

\section{APPROVED:}

Dissertation Committee:

\author{
Major Professor Tetsu Hara \\ Isaac Ginis \\ Jason M. Dahl \\ Peter P. Sullivan \\ Fabrice Veron \\ Brenton Deboef \\ DEAN OF THE GRADUATE SCHOOL
}

\section{UNIVERSITY OF RHODE ISLAND}

2020 


\begin{abstract}
There is a growing need to improve the prediction and parameterization of momentum, energy, heat, and gas exchanges between the ocean and atmosphere. It is well known that the coupling between turbulent wind and a spectrum of waves significantly modifies turbulent air-sea fluxes, thereby playing an important role in the development of wave, weather, and climate patterns. Due to the limitations of field measurements, especially in high-to-extreme wind conditions such as tropical cyclones, constraining air-sea fluxes in numerical wave, storm surge, weather, and climate forecast models continues to be a challenge due to our incomplete understanding of near-surface turbulence in the air and water that arises from complex surface wave dynamics in a range of environmental conditions.

Coupled ocean-atmosphere models do not typically resolve dynamics at surface wave scales, relying on simplified representations (or bulk parameterizations) of momentum and scalar transfer coefficients that often do not explicitly account for the sea state (wave field) and its impacts. The aim of this dissertation is to demonstrate the fidelity of large eddy simulation (LES) in representing turbulence in the wave boundary layer, and investigate the impacts on important air-sea fluxes with a focus on steep, strongly forced wind waves. In particular, we use LES to investigate the occurrence and behavior of a process known as airflow separation, which has been pinpointed as an important process affecting the observed saturation
\end{abstract}


(and potential decrease) of the drag coefficient (air-sea momentum flux) in high-toextreme wind conditions.

In Chapter 1, we use LES of airflow over sinusoidal waves to investigate the causes and features of airflow separation over strongly forced (young) wind waves. Flow fields previously observed over laboratory waves are compared with those in LES under similar conditions (see Husain et al. 56). Many of the same flow features are observed, including strong cross-wave vorticity detached from the leeward crest followed by enhanced TKE aloft, and strong wind shear near the height of the wave crest, with weak velocity, recirculation and reduced TKE in the trough-features of airflow separation over moving waves. This study demonstrates fidelity of LES to represent turbulence in the airflow above strongly forced waves. It also highlights the important wave features that modulate airflow separation and its impact on turbulence in the wave boundary layer, including the alteration of the mean wind speed, drag coefficient, and wave growth rate.

Chapters 2 and 3 expand on the previous chapter by using the identical LES approach to investigate airflow turbulence over an extended range of sea states when waves are misaligned with the wind - a frequent occurrence in the open ocean, especially under transient high wind conditions coupled to complex seas. Despite the ubiquity of this scenario, our knowledge on the impacts of turbulent airflow in such conditions remain limited. In the following two chapters, we investigate misaligned wind and waves in the context of key air-sea momentum fluxes, similar to Chapter 1.

In Chapter 2, we employ LES for turbulent airflow over steep sinusoidal waves of a range of wave phase speeds (i.e., wave ages) both following and opposing the wind. We find that while the airflow dynamics and impacts rapidly evolve as the 
wave age increases for waves following wind, there is a rather smooth transition from the slowest waves following wind, to those opposing wind, to the fastest waves opposing wind. We see increasingly stronger flow perturbations as the opposing wave phase speed increases, suggesting that airflow is separating intermittently (or exhibiting separation-like patterns). This process alters the phase averaged wind and pressure fields, causing both the wave decay rate and effective roughness length (drag coefficient) to increase rapidly.

Finally, in Chapter 3, we employ LES for wind blowing over steep, strongly forced waves following and opposing oblique wind $\left(\theta=45^{\circ}\right)$ to elucidate the impacts on wind speed and direction, drag coefficient, and wave growth/decay. We find that oblique winds maintain a signature of airflow separation while introducing a cross-wave component that strongly modifies the overall flow fields. The direction of the mean wind speed and wind shear deviate from the direction of wind stress in the wave boundary layer, especially towards the surface. Wave growth and decay rates fall within the range of typical parameterizations. The most interesting finding of this study is the erasure of the effect of waves on the effective surface roughness (drag coefficient) in oblique wind.

This dissertation emphasizes the important role of surface waves in modifying airflow within the wave boundary layer and the evolution of the wave field in space and/or time. We demonstrate that LES can be used to investigate wave-driven turbulent processes in the air over complex seas, and discuss the implications of our findings for elucidating the impacts on sea-state dependent air-sea flux parameterizations and turbulence closure models for numerical forecasts. 


\section{ACKNOWLEDGEMENTS}

First, I would like to thank my advisor Dr. Tetsu Hara for his guidance, insight, and passion. You've done so much to ensure I was supported in accomplishing what I set out to do, and I am so grateful to you.

Thank you to all the members of my committee: Dr. Peter P. Sullivan, Dr. Fabrice Veron, Dr. Jason M. Dahl, and Dr. Isaac Ginis. Peter, thank you for sharing your expertise and insight with me. Fabrice, thank you and Dr. Marc Buckley for continuing to help me understand our research efforts better from the observational side. Jason, thank you for teaching my Hydrodynamics group how to use your PIV system for our final project!

A big thank you to all of the people at URI who have been such supportive mentors and role models and have significantly contributed to my personal growth over the past five years: Dr. Kendall Moore, Dr. Jaime Palter, Dr. Kathleen Donohue, Dr. David C. Smith, Danielle Dirocco, Dr. Sunshine Menezes, and so many more. I appreciate the time I spent getting to know members of the Hara/Ginis Lab, the GSO community, and various others I've come to know at URI and in the state of Rhode Island who have come from all sorts of backgrounds and many of whom have become good friends of mine. A special thank you to Xuanyu Chen.

Lastly, thank you to my family and friends who have been around since before 
graduate school. Stephen, what would I do without you? I know Steppho is out there living his best life, waddling around honking for the liberation of his comrades. Thank you to my sisters, Aliya and Nadia. I would not have accomplished any of this work without the unwavering encouragement and support of my father. Abba and Amma, this work is dedicated to you. 


\section{PREFACE}

This dissertation was written in accordance with the manuscript format guidelines established by the Graduate School of the University of Rhode Island. The dissertation includes the following three manuscripts:

1. "Boundary Layer Turbulence over Surface Waves in a Strongly Forced Condition: LES and Observation" published in Journal of Physical Oceanography.

2. "Wind turbulence over misaligned surface waves and air-sea momentum flux. Part I: Waves following and opposing wind" prepared for submission to Journal of Physical Oceanography.

3. "Wind turbulence over misaligned surface waves and air-sea momentum flux. Part II: Waves in oblique wind" prepared for submission to Journal of Physical Oceanography. 


\section{TABLE OF CONTENTS}

$\begin{array}{ll}\text { ABSTRACT ii } & \text { ii }\end{array}$

ACKNOWLEDGEMENTS V v

PREFACE vii

TABLE OF CONTENTS viii

LIST OF TABLES - xi

LIST OF FIGURES xiii

1 Boundary Layer Turbulence over Surface Waves in a Strongly Forced Condition: LES and Observation 1

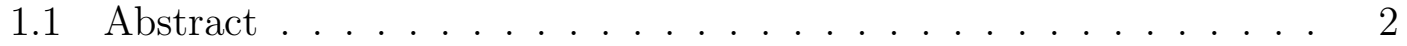

1.2 Introduction . . . . . . . . . . . . . . . . 3

1.3 Methods ...................... 6

Laboratory observation . . . . . . . . . . . . . . 6

Large eddy simulation . . . . . . . . . . . . . . . . 9

Wave-following mapping and triple decomposition . . . . . . . . . . 12

Matching experimental conditions . . . . . . . . . . . . . 15

1.4 Results and discussion . . . . . . . . . . . . . . . . . 17 
Results in physical coordinates . . . . . . . . . . . . . 17

Results in mapped coordinates . . . . . . . . . . . . 25

Momentum flux budget . . . . . . . . . . . . . . . . 28

Surface stresses and wave growth rate . . . . . . . . . . 31

Wave effect on equivalent surface roughness $\ldots \ldots \ldots \ldots$

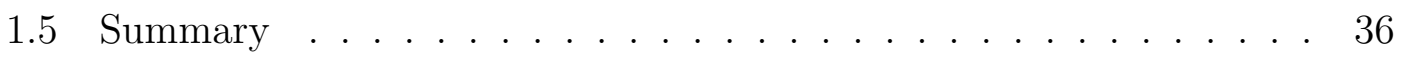

1.6 Acknowledgements . . . . . . . . . . . . . . . . . . . . . 39

1.7 Figures and Tables . . . . . . . . . . . . . . . . . 41

2 Wind turbulence over misaligned surface waves and air-sea momentum flux. Part I: Waves following and opposing wind 49

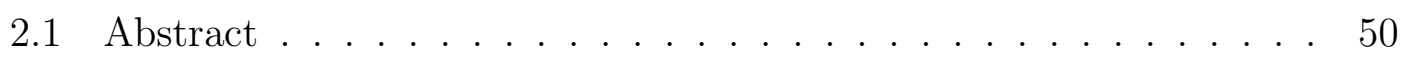

2.2 Introduction . . . . . . . . . . . . . . 50

2.3 Methods . . . . . . . . . . . . . . . . . 55

Large eddy simulation setup . . . . . . . . . . . . . 55

Wind forcing $\ldots \ldots \ldots \ldots \ldots \ldots \ldots$

Subgrid scale and surface roughness parameterizations $\ldots \ldots .58$

Simulations . . . . . . . . . . . . . . . 60

Data analysis . . . . . . . . . . . . . . . 6 61

2.4 Results and discussion $\ldots \ldots \ldots \ldots \ldots$

Two-dimensional phase averaged airflow above waves following wind 63

Two-dimensional phase averaged airflow above waves opposing wind 66

Instantaneous airflow features $\ldots \ldots \ldots$. . . . . . . . . 69

Vertical profiles of horizontally averaged wind fields . . . . . . 70

Momentum budget in mapped coordinate . . . . . . . . . . 75

Energy budget and turbulence closure parameterization . . . . . 78 
Wave growth/decay rate and equivalent roughness length . . . . . . 80

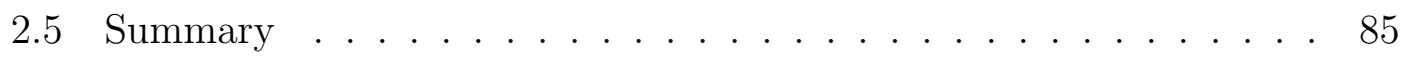

2.6 Acknowledgements . . . . . . . . . . . . . . . . . 88

2.7 Figures and Tables . . . . . . . . . . . . . . . . . . . . 88

\section{Wind turbulence over misaligned surface waves and air-sea mo-} mentum flux. Part II: Waves in oblique wind 98

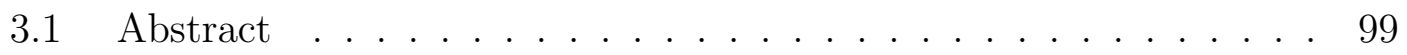

3.2 Introduction . . . . . . . . . . . . . . . . 100

3.3 Methods .......................... 102

LES setup . . . . . . . . . . . . . . . . . . . 102

Data analysis ..................... . . 104

3.4 Results and discussion . . . . . . . . . . . . . . 105

2D phase-averaged airflow . . . . . . . . . . . . 105

Horizontally averaged wind profiles in mapped coordinates . . . . . 110

Horizontally averaged momentum budget in along-wave and crosswave directions . . . . . . . . . . . . . . . . 114

Energy budget and turbulence closure parameterization . . . . . . 116

Wave growth/decay rate . . . . . . . . . . . . . . . 119

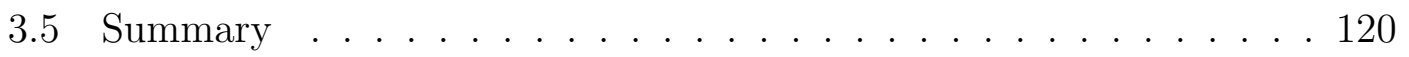

3.6 Acknowledgements . . . . . . . . . . . . . . . . . 122

3.7 Figures and Tables . . . . . . . . . . . . . . . 123

$\begin{array}{ll}\text { References } & 131\end{array}$ 


\section{LIST OF TABLES}

1.1 Non-dimensional parameters used in eleven LES experiments: wave age $\left(c / u_{* s}\right)$, wave steepness $(k a)$, surface roughness $\left(k z_{o}\right)$, and surface drift velocity $\left(u_{d} / u_{* s}\right)$. In the text and figure captions, surface roughness $k z_{o}=2.70 \times 10^{-3}, 1.35 \times 10^{-3}$, and $0.67 \times 10^{-3}$ are referred to as $k z_{o 1}, k z_{o 2}$, and $k z_{o 3}$, respectively. For Run $3 \mathrm{c}, k z_{o 3 n e w}=k z_{o 3}$

from $k x=\frac{\pi}{2}$ to $3 \frac{\pi}{2} . \ldots \ldots \ldots \ldots$

1.2 Values of non-dimensional wave growth coefficient $c_{\beta}$ for both wind forcing cases $\left(c / u_{* s}=1.4\right.$ on top; $c / u_{* s}=1.6$ on bottom). The values for $c_{\beta t o t}, c_{\beta t}, c_{\beta n}$, and $c_{\beta p}$ refer to total, tangential stress, normal stress, and pressure contributions of $c_{\beta}$. For references to runs, refer to Table $1 \ldots \ldots \ldots$. . . . . . . . . . . . 42

1.3 Values of normalized equivalent surface roughness $k z_{o e}$ for both wind forcing cases $\left(c / u_{* s}=1.4\right.$ on top; $c / u_{* s}=1.6$ on bottom). The value of $z_{o, e} / z_{o}$ is the ratio of the equivalent surface roughness $z_{o, e}$ to the parameterized surface roughness $z_{0}$. For references to runs, refer to

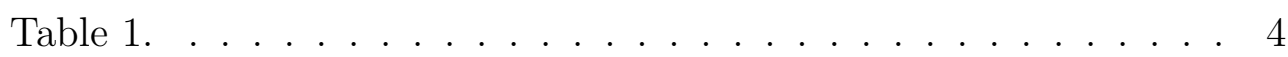


2.1 List of run conditions and results of roughness enhancement $z_{o} / z_{o b}$ and non-dimensional wave growth/decay coefficient $c_{\beta}$ for eleven LES simulations. The symbol $f$ and $o$ in the run name represents waves following and opposing wind, respectively. The symbol s in the run name represents Stoke waves. Non-dimensional parameters used in LES cases include wave age $\left(c / u_{* s}\right.$, which is negative for wind opposing wind), wave steepness ( $a k)$, and parameterized background roughness length $\left(k z_{o b}\right)$. Wave steepness is held constant for all simulations at $a k=0.2665(a k \sim 0.27)$. Parameterized background roughness is also held constant at $k z_{o b}=2.70 \times 10^{-3}$. The values for $c_{\beta t}, c_{\beta n}$, and $c_{\beta p}$ refer to tangential turbulent stress, normal turbulent stress, and pressure contributions to $c_{\beta}$, and $c_{\beta t o t}$ is the total. $\quad \ldots 89$

3.1 List of run conditions and results of roughness enhancement $z_{o} / z_{o b}$ and non-dimensional wave growth/decay coefficient $c_{\beta}$ for four LES simulations. The symbol $f$ and $o$ in the run name represents waves following and opposing wind, respectively; the subscript '45' represents oblique wind runs. The values for $c_{\beta t}, c_{\beta n}$, and $c_{\beta p}$ refer to tangential turbulent stress, normal turbulent stress, and pressure contributions to $c_{\beta}$, and $c_{\beta t o t}$ is the total. . . . . . . . . 123 


\section{LIST OF FIGURES}

1.1 Schematic of the wave-following mapping used for analysis of both LES and PIV results introduces a vertical coordinate $\zeta$ that follows the wave shape at $\zeta=0$ and gradually approaches the Cartesian $z$ coordinate as $\zeta$ increases. This mapping enables analyses below the wave crest level. The vertical momentum flux is defined using a Cartesian velocity $u$ and a contravariant velocity $W$ as shown in the small box. . . . . . . . . . . . . . . . . . 43

1.2 Phase-averaged flow fields from left to right: horizontal velocity, twodimensional turbulent kinetic energy, dissipation rate, and pressure. Each panel is labeled corresponding to its run number (see Table 1). Observations are shown on the bottom panel. All fields are nondimensionalized using surface friction velocity $\left(u_{* s}\right)$, surface stress $\left(\tau_{s}\right)$ and wavenumber $(k) . \ldots \ldots . \ldots . \ldots 44$ 
1.3 Mean normalized wind speed (a), wind shear (b), and 2D turbulent kinetic energy (c) for $c / u_{* s}=1.6$ (top panel) and $c / u_{* s}=1.4$ (bottom panel). Black lines show observational (PIV) results, while dark blue lines show LES results for three different surface roughness lengths: $k z_{o 1}$ (solid), $k z_{o 2}$ (dashed), and $k z_{o 3}$ (dotted). Perturbations include runs $2 \mathrm{~b}$ (green), $3 \mathrm{~b}$ (orange), and 3c (purple). All quantities are normalized using the surface friction velocity $u_{* s}$. Grey lines (LES: solid; PIV: dashed) in the middle panels refer to the unperturbed wind shear profile (without waves) accounting for the linear change in total wind stress with height. The results below $k \zeta \approx 0.025$ are omitted because the PIV profiles are strongly affected by viscosity and small scale waves, and above $k \zeta \approx 0.824$ due to noisiness. . . . 45

1.4 Horizontally-averaged profile of each normalized wind stress component for $c / u_{* s}=1.6$ (top panel) and $c / u_{* s}=1.4$ (bottom panel), with components shown as (a) wave-coherent stress $\left(<\tau^{w}>/ \tau_{s}\right)$ and viscous stress (pink, PIV only), (b) pressure stress $\left(<\tau^{p}>/ \tau_{s}\right)$ and vertically integrated pressure gradient $\left(\frac{\partial P}{\partial x} \zeta / \tau_{s}\right.$; light grey), (c) turbulent stress $\left(\left(<\tau^{t}>/ \tau_{s}\right)\right.$ and linearly decreasing total wind stress $\left(\tau_{\text {tot }} / \tau_{s}=1-\frac{k \zeta}{2 \pi} ;\right.$ light grey $), \ldots \ldots \ldots \ldots$

1.5 Normalized phase-averaged mapped fields of wave-coherent stress and excess turbulent stress (turbulent stress minus total wind stress) for LES and PIV. Each panel is labeled corresponding to its run number (see Table 1). Observations are shown in the bottom panel. All results are normalized by the surface stress, $\tau_{s}=u_{* s}^{2} . \quad \ldots \ldots 47$ 
1.6 Surface stress components for $c / u_{* s}=1.4$, with (1) pressure contribution, and (2) turbulent normal stress (above zero), and turbulent tangential stress (below zero). Dark blue lines show LES results for three different surface roughness lengths, with $k z_{o 1}$ (solid), $k z_{o 2}$ (dashed), and $k z_{o 3}$ (dotted). Perturbations include runs $2 \mathrm{~b}$ (green), 3b (orange), and 3c (purple). All results are normalized by the total wind stress, or surface friction velocity squared, $\tau^{\text {wind }}=u_{* s}^{2} \ldots \ldots$

2.1 Normalized phase-averaged flow fields in the $\xi-z$ coordinate for waves following wind $\left(c / u_{* s}=1.4,5.6,11.0\right.$; top three panels $)$ and waves opposing wind $\left(c / u_{* s}=-1.4,-5.6,-11.0\right.$; bottom three panels $)$ from left to right: streamlines, horizontal velocity $\left((\bar{u}-c) / u_{* s}\right)$, vertical velocity $\left(w / u_{* s}\right)$, and pressure $\left(\bar{p} / u_{* s}^{2}\right)$. Rightmost plots show the surface stress distribution for the normal stress $\overline{\tau_{n}} / u_{* s}^{2}$ (panels A and C, solid line is total normal stress and dotted line is pressure only) and for the tangential stress $\overline{\tau_{t}} / u_{* s}^{2}$ (panels B and D) for $\left|c / u_{* s}\right|=1.4,5.6$, and 11.0 (dark red, light orange, blue). The bottom two panels are for waves opposing wind. . . . . . . . . 90

2.2 Normalized phase-averaged flow fields in the $\xi$ - $z$ coordinate (left three panels) and the mapped $\xi-\zeta$ coordinate (right three panels) for waves following wind $\left(c / u_{* s}=1.4,5.6,11.0\right.$; top three panels $)$ and waves opposing wind $\left(c / u_{* s}=-1.4,-5.6,-11.0\right.$; bottom three panels) from left to right: turbulent kinetic energy $\left(\bar{e} / u_{* s}^{2}\right)$, dissipation rate $\left(\epsilon /\left(k u_{* s}^{3}\right)\right)$, and spanwise vorticity $\left(\bar{\omega}_{y} /\left(k u_{* s}\right)\right) \ldots \ldots \ldots 1$ 
2.3 Normalized instantaneous vorticity fields $\left(\omega_{y} /\left(k u_{* s}\right)\right)$ in the $x-z$ coordinate for waves following wind $\left(c / u_{* s}=1.4,11.0\right.$; top two panels) and waves opposing wind $\left(c / u_{* s}=-1.4,-11.0\right.$; bottom two panels)

2.4 Normalized vertical profiles of horizontally averaged wind speed $\left(\langle u\rangle / u_{* s}^{2}\right)$, wind shear $\left(\frac{\partial\langle u\rangle}{\partial \zeta} \frac{\kappa \zeta}{u_{* s}}\right)$, and TKE $\left(\langle\bar{e}\rangle / u_{* s}^{2}\right)$ for waves following wind (top panel) and waves opposing wind (bottom panel) for $\left|c / u_{* s}\right|=1.4,2.8,5.6,8.2$, and 11.0 (dark red, dark orange, light orange, light green, and blue). The dot-dashed dark red line is the Stokes wave case for $c / u_{* s}=1.4 \ldots \ldots \ldots \ldots$. . . . . 93

2.5 On the left: normalized phase-averaged fields of wave-coherent stress $\left(\overline{\tau^{w}} / u_{* s}^{2}=\tilde{u} \tilde{W} / u_{* s}^{2}\right)$ and turbulent stress $\left(\overline{\tau^{t}} / u_{* s}^{2}=\overline{u^{\prime} W^{\prime}} / u_{* s}^{2}\right)$ in the mapped $\xi-\zeta$ coordinate for waves following wind $\left(c / u_{* s}=1.4\right.$, 5.6, 11.0; top three panels $)$ and waves opposing wind $\left(c / u_{* s}=\right.$ $-1.4,-5.6,-11.0$; bottom three panels). On the right: normalized vertical profiles of horizontally averaged momentum budget terms for waves following wind (top panels) and waves opposing wind (bottom panels), including the total wind stress $\left(\left\langle\tau^{\text {tot }}\right\rangle / u_{* s}^{2}\right)$, pressure stress $\left(\left\langle\tau^{p}\right\rangle / u_{* s}^{2}\right)$, turbulent stress $\left(\left\langle\tau^{t}\right\rangle / u_{* s}^{2}\right)$, and wave-coherent stress $\left(\left\langle\tau^{w}\right\rangle / u_{* s}^{2}\right)$ for $\left|c / u_{* s}\right|=1.4,2.8,5.6,8.2$, and 11.0 (dark red, dark orange, light orange, light green, and blue). The dot-dashed dark red line is the Stokes wave case for $c / u_{* s}=1.4 \ldots \ldots$. . . . . 94 
2.6 Left plots: normalized vertical profiles of horizontally averaged energy budget terms (the first, second, third and fourth terms of Equation 3.3 are solid grey, solid, dot-dashed, and dotted lines, respectively) with thin dotted lines near zero equalling the sum of all energy budget terms. Middle plots: normalized vertical profiles of horizontally averaged turbulent stress $\left(\left\langle\tau^{t}\right\rangle / u_{* s}^{2}\right.$, also shown in Figure 2.5). Right plots: normalized vertical profiles of eddy viscosity $\left(K /\left(\kappa u_{* s} \zeta\right)\right)$. For the left, middle, and right plots, the thin grey lines represent mean shear production, turbulent stress, and eddy viscosity profiles over a flat wall with linearly decreasing wind stress (solid) and constant stress (dashed) with respect to $k \zeta$. Results for waves following (opposing) wind are shown on the top (bottom) panel for $\left|c / u_{* s}\right|=1.4,5.6$, and 11.0 (dark red, light orange, and blue). . . . 95

2.7 Top plot: wave growth/decay coefficient $\left|c_{\beta}\right|$ for waves following wind (black lines) and for waves opposing wind (red lines) as a function of $\left|c / u_{* s}\right|$. Thin solid lines represent the contribution of the total normal stress $\left(c_{\beta p}+c_{\beta n}\right)$, dotted lines represent the pressure contribution $\left(c_{\beta p}\right)$, dashed lines represent tangential stress contribution $\left(c_{\beta t}\right)$, and thick solid lines represent the sum of all components $\left(c_{\beta t o t}=\right.$ $\left.c_{\beta p}+c_{\beta n}+c_{\beta t}\right)$. Bottom plot: ratio of the equivalent surface roughness to the background (parameterized) surface roughness $z_{o} / z_{o b}$ as a function of $\left|c / u_{* s}\right|$ for waves following wind (black line) and waves opposing wind (red line). In both plots, the dark grey squares represent the Stokes wave case for $c / u_{* s}=1.4$ for $c_{\beta t o t}$ (large square)

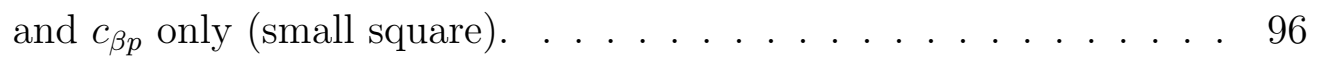


2.8 Top plot: Comparison of wave decay coefficient $\left|c_{\beta}\right|$ as a function of wave age $\left|c / u_{* s}\right|$ for the current LES study (thick red line: total $c_{\beta t o t}$, thin red line: only $c_{\beta p}$ ), the wall-resolved LES results of Cao et al. [16] (blue squares), the parameterization of Mitsuyasu and Yoshida [70] (dashed line), and the results of Harris et al. [52] (dot-dashed line) and Cohen [20] (dotted line). Middle plot: Comparison of wave decay rate $|\beta / \omega|$ as a function of inverse wave age $\left|u_{* s} / c\right|$ with lines corresponding to those of the top plot. Compare with Cao et al. [16] Figure 18. Bottom plot: Comparison of wave decay rate $\beta / \omega$ as a function of $\left(\left\langle u_{\lambda / 2}\right\rangle / c-1\right)\left|\left\langle u_{\lambda / 2}\right\rangle / c-1\right|$, including the current LES results (thick red line: total decay rate, thin red line: pressure contribution only), the wall-resolved LES of Cao et al. [16] (blue squares), the parameterization of Mitsuyasu and Yoshida [70] (dot-dashed line), and the observational results and parameterization of Donelan [25] (black circles and black line, respectively). Compare to Figure 19 of Cao et al. [16]. . . . . . . . . . . . . . . . . . . 97 
3.1 Normalized phase-averaged flow fields in the $\xi$ - $z$ coordinate for waves following wind (top two rows; oblique wind in second row) and for waves opposing wind (bottom two rows; oblique wind in fourth row). From left to right: along-wave velocity $\left((\bar{u}-c) / u_{* s}\right)$, crosswave velocity $\left(v / u_{* s}\right)$, vertical velocity $\left(w / u_{* s}\right)$, and pressure $\left(\bar{p} / u_{* s}^{2}\right)$. Rightmost plots show the surface stress distribution for the normal stress $\overline{\tau_{n}} / u_{* S}^{2}$ (panels A and $\mathrm{C}$, solid line is total normal stress and dotted line is pressure only) and for the tangential stress $\overline{\tau_{t}} / u_{* s}^{2}$ in the $x$ direction (panels $\mathrm{B}$ and $\mathrm{D}$ ) for waves following wind (orange lines, top two panels) and opposing wind (blue lines, bottom two panels) in aligned (thick lines) and oblique (thin lines) wind conditions.124

3.2 Normalized phase-averaged flow fields in the $\xi-z$ coordinate (left three panels) and the mapped $\xi-\zeta$ coordinate (right three panels) for waves following wind (top two rows; oblique wind in second row) and for waves opposing wind (bottom two rows; oblique wind in fourth row). From left to right: turbulent kinetic energy $\left(\bar{e} / u_{* s}^{2}\right)$, dissipation rate $\left(\epsilon /\left(k u_{* s}^{3}\right)\right)$, and vorticity magnitude $\left(\bar{\omega}_{h} /\left(k u_{* s}\right)\right) . \quad .125$

3.3 Normalized phase-averaged flow fields in the $\xi$ - $z$ coordinate (left three panels) and the mapped $\xi-\zeta$ coordinate (right three panels) for waves following wind (top two rows; oblique wind in second row) and for waves opposing wind (bottom two rows; oblique wind in fourth row). From left to right: vorticity magnitude $\left(\bar{\omega}_{h} /\left(k u_{* s}\right)\right)$, cross-wave vorticity $\left(\bar{\omega}_{y} /\left(k u_{* s}\right)\right)$, and along-wave vorticity $\left(-\bar{\omega}_{x} /\left(k u_{* s}\right)\right) . \ldots 125$ 
3.4 Normalized phase-averaged flow fields in the $\xi$ - $z$ coordinate (left three panels) and the mapped $\xi-\zeta$ coordinate (right three panels) for waves following wind (top two rows; oblique wind in second row) and for waves opposing wind (bottom two rows; oblique wind in fourth row). From left to right: the along-wave component $\left(0.5 \overline{u^{\prime} u^{\prime}} / u_{* s}^{2}\right)$, the cross-wave component $\left(0.5 \overline{v^{\prime} v^{\prime}} / u_{* s}^{2}\right)$, and the vertical component $\left(0.5 \overline{w^{\prime} w^{\prime}} / u_{* s}^{2}\right)$ of TKE. . . . . . . . . . . . . . 12

3.5 Top row from left to right: normalized vertical profiles of horizontally averaged along-wave wind speed $\left(\langle u\rangle / u_{* s}\right)$, cross-wave wind speed $\left(\langle v\rangle / u_{* s}\right)$, wind speed magnitude, and wind speed angle. Middle row from left to right: normalized vertical profiles of horizontally averaged along-wave wind shear $\left(\frac{\partial\langle u\rangle}{\partial \zeta} \frac{\kappa \zeta}{u_{* s}}\right)$, cross-wave wind shear $\left(\frac{\partial\langle v\rangle}{\partial \zeta} \frac{\kappa \zeta}{u_{* s}}\right)$, wind shear magnitude, and wind shear angle. In panel $(\mathrm{k})$ dashed lines show angle of horizontally averaged turbulent stress vector $\left(\left\langle\tau_{13}^{t}\right\rangle,\left\langle\tau_{23}^{t}\right\rangle\right)$. Bottom row from left to right: normalized vertical profiles of horizontally averaged TKE magnitude $\left(\langle\bar{e}\rangle / u_{* s}^{2}\right)$, the along-wave component $\left(0.5\left\langle\overline{u^{\prime} u^{\prime}}\right\rangle / u_{* s}^{2}\right)$, the cross-wave component $\left(0.5\left\langle\overline{v^{\prime} v^{\prime}}\right\rangle / u_{* s}^{2}\right)$, and the vertical component $\left(0.5\left\langle\overline{w^{\prime} w^{\prime}}\right\rangle / u_{* s}^{2}\right)$ of the TKE. In all panels profiles for waves following (opposing) aligned wind are shown as thick orange (blue) lines, and profiles for waves following (opposing) oblique wind are shown as thin orange (blue) lines. Grey lines show profiles for flat wall cases (explained in the

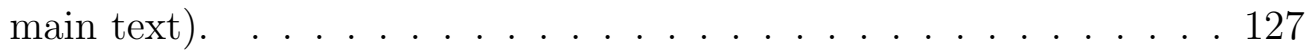


3.6 Normalized vertical profiles of horizontally averaged momentum budget terms following wind (orange lines) and opposing wind (blue lines) for oblique (thin lines) and aligned (thick lines) wind conditions. In the top row, the along-wave $(x)$ momentum budget includes total wind stress in the $x$-direction $\left(\left\langle\tau_{13}^{\text {tot }}\right\rangle / u_{* s}^{2}\right)$, pressure stress $\left(\left\langle\tau_{13}^{p}\right\rangle / u_{* s}^{2}\right)$, along-wave turbulent stress $\left(\left\langle\tau_{13}^{t}\right\rangle / u_{* s}^{2}\right)$, and along-wave wave-coherent stress $\left(\left\langle\tau_{13}^{w}\right\rangle / u_{* s}^{2}\right)$. In the bottom row, the cross-wave (y) momentum budget includes total wind stress in the $y$-direction $\left(\left\langle\tau_{23}^{t o t}\right\rangle / u_{* s}^{2}\right)$, cross-wave turbulent stress $\left(\left\langle\tau_{23}^{t}\right\rangle / u_{* s}^{2}\right)$, and cross-wave wave-coherent stress $\left(\left\langle\tau_{23}^{w}\right\rangle / u_{* s}^{2}\right)$. Grey lines show profiles for flat wall cases (explained in the main text). . . . . . . . . . . 128

3.7 Top four panels: Normalized phase-averaged fields of the along-wave turbulent stress $\left(\overline{\tau_{13}^{t}} / u_{* s}^{2}=\overline{u^{\prime} W^{\prime}} / u_{* s}^{2}\right)$, cross-wave turbulent stress $\left(\overline{\tau_{23}^{t}} / u_{* s}^{2}=\overline{v^{\prime} W^{\prime}} / u_{* s}^{2}\right)$, along-wave wave-coherent stress $\left(\overline{\tau_{13}^{w}} / u_{* s}^{2}=\right.$ $\left.\tilde{u} \tilde{W} / u_{* s}^{2}\right)$, and cross-wave wave-coherent stress $\left(\overline{\tau_{23}^{w}} / u_{* s}^{2}=\tilde{v} \tilde{W} / u_{* s}^{2}\right)$ in the mapped $\xi-\zeta$ coordinate. From top to bottom: waves following aligned wind, waves following oblique wind, wave opposing aligned wind, and waves opposing oblique wind. Bottom four panels: Normalized phase-averaged fields of the wave-coherent vertical velocity $\left(\tilde{W} / u_{* s}\right)$, along-wave velocity $\left(\tilde{u} / u_{* s}\right)$, and cross-wave velocity $\left(\tilde{v} / u_{* s}\right)$ in the mapped $\xi-\zeta$ coordinate. 
3.8 Normalized vertical profiles of horizontally averaged energy budget terms. The first, second, third and fourth terms of Equation 3.3 are dark green, dark red, light green, and blue lines, respectively. Thick (thin) lines are for aligned (oblique) wind. Solid black lines are sum of all energy budget terms. Top (bottom) panel is for waves following (opposing) wind. . . . . . . . . . . . . . . . 130 
Boundary Layer Turbulence over Surface Waves in a Strongly Forced Condition: LES and Observation

Nyla Husain ${ }^{1 *}$, Tetsu Hara ${ }^{1}$, Marc P. Buckley², Kianoosh Yousefi ${ }^{3}$, Fabrice Veron $^{3}$, and Peter P. Sullivan ${ }^{4}$

Published in Journal of Physical Oceanography, August 2019.

\footnotetext{
${ }^{1}$ Graduate School of Oceanography, University of Rhode Island, Narragansett, Rhode Island *Corresponding author email: nylahusain@uri.edu

${ }^{2}$ Institute of Coastal Research, Helmholtz-Zentrum Geesthacht, Geesthact, Germany

${ }^{3}$ College of Earth, Ocean, and Environment, University of Delaware, Newark, Delaware

${ }^{4}$ National Center for Atmospheric Research, Boulder, Colorado
} 


\subsection{Abstract}

The impact of sea state on air-sea momentum flux (or wind stress) is a poorly understood component of wind-wave interactions, particularly in high wind conditions. Wind stress and mean wind profile over the ocean are influenced by the characteristics of boundary layer turbulence over surface waves, which is strongly modulated by transient airflow separation events; however, the features controlling their occurrence and intensity are not well known. A large-eddy simulation (LES) for wind over a sinusoidal wave train is employed to reproduce laboratory observations of phase-averaged airflow over waves in strongly forced conditions. The LES and observation both use a wave-following coordinate system with a decomposition of wind velocity into mean, wave-coherent, and turbulent fluctuation components. The LES results of the mean wind profile and structure of wave-induced and turbulent stress components agree reasonably well with observations. Both LES and observation show enhanced turbulent stress and mean wind shear at the height of the wave crest, signifying the impact of intermittent airflow separation events. Disparities exist particularly near the crest, suggesting that airflow separation and sheltering are affected by the nonlinearity and unsteadiness of laboratory waves. Our results also suggest that the intensity of airflow separation is most sensitive to wave steepness and the surface roughness parameterization near the crest. These results clarify how the characteristics of finite amplitude waves can control the airflow dynamics, which may substantially influence the mean wind profile, equivalent surface roughness, and drag coefficient. 


\subsection{Introduction}

The coupling between wind and surface waves influences marine weather and climate by controlling the exchange of momentum, heat, and gases at the air-sea interface. Coupled ocean-atmosphere models have typically relied on a bulk parameterization of wind stress (or drag coefficient), which assumes a relationship with the neutral wind speed at 10 meters above the sea surface. This simplification does not account for the effects of different sea states; in particular, it ignores intermittent and nonlinear wave-induced turbulent processes such as airflow separation and wave breaking. These have been shown to modify the local wind stress and surface roughness, causing deviations from the assumed logarithmic wind profile under varying wind-wave conditions Banner [4], Banner and Melville [5].

Many previous modeling studies explored how different sea states (surface wave spectra) affect the drag coefficient (Hara and Belcher 49, 50, Makin and Kudryavtsev $66)$, including some studies that explicitly accounted for the airflow separation effects (Kudryavtsev and Makin 57, Kudryavtsev et al. 61, Kukulka and Hara 62, Mueller and Veron 76). More recent studies considered the effects of complex sea states under tropical cyclones (Donelan et al. 28, Moon et al. 73, 74, Reichl et al. 83), including the impact of sea spray from breaking waves (Richter and Sullivan 85, Veron 102). However, uncertainty remains as to the observed relationship between the sea-state and wind stress, especially under high wind conditions in which non-linear turbulent processes dominate the air-sea exchange (Black et al. 9, Edson et al. 36, Holthuijsen et al. 55). It is apparent that these processes, which occur ubiquitously in the open ocean, produce cumulative effects on the drag coefficient and structure of the marine atmospheric boundary layer, but it is not 
clear how they manifest under different surface wave conditions. In fact, airflow separation has been viewed as a process which both limits form (pressure) drag in steep, non-breaking wave conditions, and enhances the drag in breaking wave conditions (Donelan 26, Grare et al. 47, Peirson and Garcia 79). The contribution of the form drag to the total stress has been found to increase from incipient to active breaking waves (Banner 4, Sullivan et al. 90), but it is still unclear what role airflow separation plays in this transition.

Airflow separation has been well-documented over breaking waves (Banner and Melville 5, Reul et al. 84) and has been observed intermittently over strongly forced non-breaking waves using Particle Image Velocimetry (PIV) in a wind-wave flume (e.g., Buckley and Veron 12, Troitskaya et al. 99, Veron et al. 103), large eddy simulation (e.g., Sullivan et al. 90), and direct numerical simulation (Yang and Shen 107). It is characterized by a thin layer of enhanced shear and spanwise vorticity that detaches at the wave crest, generating turbulence and enhanced dissipation downstream. This separation often produces a low velocity recirculation cell, or "bubble," on the lee side of the wave. Beyond these features, the airflow reattaches on the windward side of the following wave crest. Because the separation happens intermittently and fluctuates in size and intensity, the mean (phase-averaged) flow field does not show a clear flow separation pattern. Instead, the size of the recirculation cell of the mean flow following the phase speed increases as flow separation events become more frequent.

While the effect is seen in a range of sea states, the wave characteristics controlling its occurrence and intensity remain elusive. For example, it has been indicated that the wave steepness controls the magnitude of form drag (Grare et al. 47, Peirson and Garcia 79, Sullivan et al. 90), but its effects on airflow separation 
processes that modulate the form drag are not well known. The surface roughness due to small-scale waves and breakers may also be connected to the occurrence of airflow separation over dominant waves and play a comparably important role in the form drag modulation (Donelan et al. 30, Gent and Taylor 44, Kudryavtsev and Makin 58, Taylor and Yelland 96).

In Hara and Sullivan [51], a large eddy simulation (LES) for wind over a sinusoidal wave train was employed to investigate how airflow dynamics are coupled to finite amplitude, strongly-forced wind-waves. The study revealed a signature of airflow separation characterized by enhanced turbulence above a recirculation cell on the lee side of the wave crest, accompanied by enhanced downward turbulent stress and mean wind shear at a similar elevation. It also confirmed the presence of large pressure form drag, reduction of turbulent stress and reduced mean wind shear closer to the surface. Variations in the mean wind profile due to turbulence in the airflow ultimately determine the equivalent surface roughness, or the drag coefficient modified by the surface waves.

In Buckley and Veron [13, 14], high resolution PIV experiments enabled measurements of airflow dynamics very close to the surface under a range of wind-wave conditions. Instantaneous airflow separation patterns in observations resembled those exhibited by LES under similar conditions, showing promise that the airflow dynamics could be reproduced and their effects explored further in LES.

The present study seeks to determine to what extent LES can reproduce the observed airflow dynamics induced by strongly forced wind-waves using horizontal mean and wave phase-averaged flow fields. By exploring the range of airflow patterns when wind-wave characteristics are altered, we aim to elucidate how finite amplitude surface waves modify the mean wind profile, effective surface roughness, 
and drag coefficient.

\subsection{Methods}

\section{Laboratory observation}

The measurements of airflow over wind-waves used in this study are part of the extensive dataset acquired by Buckley [11] (see also Buckley and Veron 12, Buckley and Veron 13, and Buckley and Veron 14). A full description of the experimental set-up, methods, and results are described in Buckley and Veron [13]. These data were taken using a high resolution PIV system in a wind-wave flume measuring 42 $\mathrm{m}$ long, $1 \mathrm{~m}$ wide, and $1.25 \mathrm{~m}$ high with a water depth of $0.70 \mathrm{~m}$. The recirculating wind tunnel blew air directly over the water surface, and air was seeded with $O(10 \mu \mathrm{m})$ water droplets produced by a fog generator located at zero fetch. These droplets acted as Lagrangian tracers that moved with the airflow.

The PIV set-up was located at a fetch of $22.7 \mathrm{~m}$ where wind-waves had sufficiently developed. The PIV system measured along-channel 2D velocity fields in the midsection of the flume by illuminating water particles with a high intensity green laser sheet. By measuring the displacement of groups of water particles at a specified time interval, the instantaneous velocity fields were computed at a resolution of $O(100 \mu \mathrm{m})$. These velocity fields enabled the analysis of $2 \mathrm{D}$ wind vectors, turbulent kinetic energy (TKE), turbulent stresses, and vorticity.

Fluorescent dye was used in the water to detect the wavy surface on the same plane as the airflow measurements by using Laser Induced Fluorescence (LIF). The illumination of the water against a dark background enabled detection of the surface waves and measurement of their properties (i.e. wavelength, frequency, and 
amplitude). A separate camera with a large field of view was used to capture waves as they entered and exited the PIV field of view. These measurements were used to detect the wave phase along the surface by applying a Hilbert transform (Buckley and Veron 13, Oppenheim and Schafer 77). Laser wave gauges also measured time series of the wave height for each PIV image. Because laboratory wind-waves are not strictly periodic, the wavenumber $k$ and the wave phase speed $c$ were determined from the observed peak frequency using the linear wave theory, and the wave amplitude $a$ was determined from the root mean square (RMS) wave amplitude.

In a wind-wave flume, wind is forced by a horizontal pressure gradient along the tank (i.e. pressure-driven channel flow). Far from the entry of the flume, where wind and wave fields vary slowly along the tank, this pressure gradient is approximately constant with height. The total wind stress (or flux) then varies linearly with height so that its vertical gradient is balanced by the horizontal pressure gradient (Uz et al. 101, Zavadsky and Shemer 111). This means that the so-called "constant-flux" layer does not exist in the tank, and the stress actually decreases linearly towards the flume ceiling. The effect of this difference is expected to be small when focusing on wind fields very close to the water surface. For this study, the vertical wind stress profile was estimated using the PIV velocity measurements above the level of surface wave crests. The observed profile suggested that the stress magnitude decreased with height and approached zero toward the top of the flume due to relatively small friction at the ceiling.

We therefore fitted a linear stress profile to the observed profile, requiring that it reaches zero at the ceiling. This profile was extrapolated towards the surface to estimate the surface wind stress and the surface friction velocity $u_{* s}$. We have 
ascertained that the results presented in this study are not sensitive to uncertainty of the stress profile in the tank, because our analyses focus on the wind field very close to the water surface.

In this study, comparison of observations with LES is performed using wave ages of $c / u_{* s}=1.4$ and 1.6 (see Table 1 ). These conditions exemplified the two most strongly forced wind-wave fields recorded in the PIV experiments. At these wave ages, waves experienced occasional breaking, producing ripples of $O(1 \mathrm{~cm})$ that visibly enhanced the surface roughness. Although airflow separation was also observed intermittently at higher wave ages $\left(c / u_{* s}=2.5\right.$ and 3.7$)$, LES experienced limitations resolving the wind field over such waves. We believe that this limitation was caused by a reduction of larger-scale turbulence associated with a lower dominant (phase-averaged) wave slope and a lower value of background surface roughness (undeveloped small scale waves) in the laboratory, making LES more sensitive to unresolved (subgrid) motions and more difficult to obtain a stable solution. We expect that surface roughness in the open ocean is significantly more pronounced than in a laboratory flume at such wave ages, and LES is suitable for simulating airflow in such conditions. In fact, past studies have successfully used identical turbulence closure schemes to explore a broader range of wave ages and wave slopes with no model stability issues (see Sullivan et al. [93] and Sullivan et al. [90]). However, in a wind wave flume such wave ages correspond to very low wind speeds with undeveloped higher frequency waves, and the viscosity effects become important near the surface. It is possible that direct numerical simulation (DNS) would be more suitable for these more mature laboratory waves (i.e. Yang and Shen [107]) as they are able to resolve all scales of turbulence and viscous effects. Since our main goal is to validate LES results against laboratory observations, we 
focus on the two low wave age conditions in this study.

It is important to note that the dominant laboratory waves of such wave ages are not common in the open ocean; that is, they are shorter and of higher frequency than typical waves near the spectral peak. However, investigating the airflow characteristics and the resulting enhancement of the drag coefficient over such waves is important, because it is well known that high frequency waves represent an important part of the wave spectrum that can support a large portion of the air-sea momentum flux (Donelan et al. 28, Reichl et al. 83).

\section{Large eddy simulation}

The LES methodology is employed using an approach identical to that of Sullivan et al. [93], Hara and Sullivan [51], and Sullivan et al. [90]. We define $t$ as time, $x$ as an along-wind coordinate, $y$ as a cross-wind coordinate, and $z$ as a vertical upward coordinate with $z=0$ at the mean water surface, with velocities $(u, v, w)$ in $(x, y, z)$ directions. In this study we consider steady wind over a periodic surface wave train with the surface specified as $z=h(x, t)=a \cos (k x-\omega t)$, where $a$ is the wave amplitude, $k$ is the wavenumber, $\omega$ is the angular frequency, and $c=\omega / k$ is the phase speed. The phase speed $c$ and the wave orbital velocities at the water surface, $(u, w)$, are specified based on the linear deep water wave theory, $c=\omega / k=\sqrt{g / k}, u=a \omega \cos (k x-\omega t)+u_{d}$ and $w=a \omega \sin (k x-\omega t)$, where $u_{d}$ is the surface drift velocity explained in the next section. Implications of using the sinusoidal wave shape and the linear wave phase and orbital velocities will be discussed later.

Turbulence in LES for wind over strongly forced waves is dominated by resolved large-scale eddies, and therefore less sensitive to subgrid-scale motion. The turbulent 
flow in LES is thus spatially filtered, and the subgrid fluxes are parameterized using a conventional TKE-closure SGS parameterization described in detail in Moeng [71], Sullivan et al. [93], and Moeng and Sullivan [72], and utilized by Hara and Sullivan [51] and Sullivan et al. [90]. It models the subgrid-scale (SGS) terms (including stress, energy flux, and energy dissipation) by relating SGS fluxes to the resolved-scale field through bulk transfer coefficients. This approach requires a roughness parameter to be imposed on the surface with a bulk aerodynamic formula for momentum (and scalars) applied point-by-point along the wavy boundary based on Monin-Obukhov (MO) similarity theory. The lower boundary is therefore parameterized with a surface roughness length $z_{o}$, which serves as a representation of the surface viscous stress effect and the roughness of smaller unresolved elements, such as higher frequency waves. This roughness length relates the local instantaneous surface tangential stress and the tangential velocity at the first LES vertical grid level, with a log wind profile assumption in between. A free slip (no tangential stress) condition is imposed at the upper boundary, where the surface is flat and vertical velocity disappears $(w=0)$.

Given the use of an SGS model in LES, the turbulent stress and TKE consist of both resolved and parameterized SGS contributions; the LES results presented in this study always combine the two. Because the high resolution (of $O(100 \mu m)$ ) PIV measurements resolve most of the turbulent eddy scales (Buckley and Veron 13), it is reasonable to include the SGS contribution in LES results when comparing with PIV.

The mapping between physical vertical coordinate, $z$, and the computational 
vertical coordinate, $\zeta^{L E S}$, is given as

$$
z=\zeta^{L E S}+h(x, t)\left(1-\frac{\zeta^{L E S}}{l_{\zeta}}\right)^{3}
$$

so that $\zeta^{L E S}$ coordinate follows the waves close to the surface, and gradually approaches the flat top boundary at $z=\zeta^{L E S}=l_{\zeta}$. The computational domain has a size of $l_{x} \times l_{y} \times l_{\zeta}$ where $l_{x}=l_{y}=5 \lambda$ and $l_{\zeta}=\lambda$, with $\lambda=2 \pi / k$ as the wavelength. It is discretized with $\left(N_{x}, N_{y}, N_{\zeta}\right)=(256,256,128)$ grid points, making the horizontal resolution $\Delta x=\Delta y=0.01953 \lambda$. Sullivan et al. [90] considered a grid spacing identical to that of the present study as well as one with four times finer horizontal resolution, finding only small differences between the coarse and fine grid results. The vertical spacing ratio is nonuniform; the ratio between neighboring cells is held constant at 1.0028, with the first point off the water surface located at $\zeta_{1}^{L E S}=0.0065 \lambda$. Waves propagate in and out of the computational domain in the positive $x$-direction with doubly periodic conditions imposed on the horizontal boundaries. Wind forcing is applied in the $x$-direction with a negative external pressure gradient $\partial P / \partial x$ that yields a negative surface stress, $\tau_{s}=(\partial P / \partial x) l_{\zeta}$, where $\tau_{s}$ is a sum of the form (pressure) stress and the viscous (subgrid) stress at the surface. The surface friction velocity is defined as $u_{* s}=\left|\tau_{s}\right|^{1 / 2}$, where both $P$ and $\tau_{s}$ are already divided by air density so that they have a dimension of velocity squared. The simulation is run for approximately 60,000 time steps and averaged over the last 20,000 time steps after the wind field has become statistically steady. Sullivan et al. [93] and Sullivan et al. [90] provide a full description of the LES algorithm and numerical methods used to solve the governing equations. 


\section{Wave-following mapping and triple decomposition}

The analyses of both LES and observation are performed in mapped wavefollowing coordinates (except for Figure 1.2), and the turbulent and wave-induced perturbations in the airflow are analyzed by decomposing a given quantity into mean, wave-coherent, and turbulent fluctuation components, as in Hara and Sullivan [51]. Previous studies have also employed a similar wave-following coordinate system to study the partition of momentum flux in the wave boundary layer (Chalikov and Rainchik 17, Sullivan et al. 92).

With the LES results, we first introduce horizontal coordinates that move with the wave:

$$
\xi=x-c t, \eta=y
$$

so that the wave shape $h=a \cos (k \xi)$ becomes steady in time. The vertical coordinate is then mapped from $z$ to $\zeta$,

$$
z=\zeta+a \cos (k \xi) e^{-k \zeta}
$$

with the Jacobian of the coordinate transformation

$$
J=\frac{\partial \zeta}{\partial z}
$$

so that $\zeta=0$ exactly follows the wave shape and $\zeta$ gradually approaches $z$ as it increases (see Figure 1.1). This mapping enables us to examine the airflow everywhere in the field, including below the wave crest level. It differs from that of the LES computational domain in that the waviness of constant $\zeta$ lines decays exponentially, while the waviness of constant $\zeta^{L E S}$ lines is forced to disappear at 
the fixed, flat top boundary. Therefore, the LES results are first mapped from the $\zeta^{L E S}$ coordinate to $z$ coordinate, and then remapped to the $\zeta$ coordinate for analysis.

In this mapped coordinate system, each LES variable, $\psi$, is averaged in both $\eta$ and $t$, and is separated into a phase average $\bar{\psi}$ and a turbulent fluctuation $\psi^{\prime}$,

$$
\psi=\bar{\psi}+\psi^{\prime}
$$

Next, the phase average $\bar{\psi}$, which is a function of $\xi$ and $\zeta$, is averaged in $\xi$ and is separated into a horizontal mean $\langle\psi\rangle$ and a wave-coherent $\widetilde{\psi}$,

$$
\bar{\psi}=\langle\psi\rangle+\widetilde{\psi},
$$

where $\langle\psi\rangle$ is a function of $\zeta$ only.

Hara and Sullivan [51] provide a full description of this triple decomposition and derive the continuity, momentum, and energy equations in the mapped coordinate system. In particular, the $x$-momentum equation, normalized by the surface wind stress $\tau_{s}$, becomes

$$
\frac{\left\langle\tau^{w}\right\rangle+\left\langle\tau^{p}\right\rangle}{\tau_{s}}+\frac{\left\langle\tau^{t}\right\rangle}{\tau_{s}}+\frac{\frac{\partial P}{\partial x} \zeta}{\tau_{s}}=1
$$

Here, the horizontal mean wave-coherent stress $\left\langle\tau^{w}\right\rangle=\langle\widetilde{u} \widetilde{W}\rangle$ and turbulent stress $\left\langle\tau^{t}\right\rangle=\left\langle u^{\prime} W^{\prime}\right\rangle$ are defined using a Cartesian velocity $u$ and a contravariant velocity

$$
W=\frac{1}{J} u \frac{\partial \zeta}{\partial x}+w
$$

which is perpendicular to a constant $\zeta$ surface. Therefore, the term $u W$ refers to flux of $x$-momentum across a constant $\zeta$ surface due to an advective velocity $W$ 
(see Figure 1.1). The pressure stress is defined as

$$
\tau^{p}=\frac{1}{J} \bar{p} \frac{\partial \zeta}{\partial x}
$$

which also transfers $x$-momentum across the tilted constant $\zeta$ surface. Equation (1.7) shows that the total wind stress is a sum of the wave-coherent stress, pressure stress, and turbulent stress, varying linearly in the presence of the pressure gradient in the tank. In LES, the parameterized subgrid-scale viscous stress, which becomes significant towards the surface, is included within the turbulent stress.

The wave shape in the instantaneous observational flow fields is neither strictly periodic nor sinusoidal. First, the vertical coordinate is mapped from $z$ to $\zeta^{\prime}$ based on the observed instantaneous surface wave shape,

$$
z=\zeta^{\prime}+\sum_{n} a_{n} \cos \left(k_{n} x+\phi_{n}\right) e^{-k_{n} \zeta^{\prime}}
$$

where $a_{n}, k_{n}$, and $\phi_{n}$ are respectively the amplitude, wavenumber, and phase of the $n$-th mode in the Fourier decomposition of the wave shape (Buckley and Veron $12,13,14)$. Note that we have differentiated the PIV mapping $\left(\xi^{\prime}, \zeta^{\prime}\right)$ from that of $\operatorname{LES}(\xi, \zeta)$. This mapping is a generalized form of the mapping of $(2.3)$ and differs from that of LES in that it accounts for multiple wave modes observed instantaneously while LES only contains one wave mode. Nevertheless, we have found that the phase-averaged wave shape from observations was very close to sinusoidal with no phase-locked higher frequency wave modes visible. The phase of each observed wave is determined by applying a Hilbert transform directly to the observed wave profile, and the location of the left (right) crest of each wave is then assigned as $k \xi^{\prime}=0\left(k \xi^{\prime}=2 \pi\right)$. Note that the $k \xi^{\prime}$ value varies linearly 
with respect to the phase detected by the Hilbert transform and is not necessarily linear with respect to $x$ due to the asymmetry of instantaneous waves about the crest. Finally, the phase speed of waves at the observed peak frequency, based on linear wave theory, is subtracted from the observed horizontal velocity so that the phase-averaged quantities are approximately consistent with those of the LES results. More details on this phase-averaging and its implications will be discussed in the results section.

\section{Matching experimental conditions}

All variables in LES are normalized by length scale $1 / k$ (inverse wavenumber), velocity scale $u_{* s}$ (surface friction velocity), and time scale $1 / k u_{* s}$. Note that the

surface wind stress (or the surface friction velocity squared, $u_{* s}^{2}$ ) is the surface value of the linearly varying total wind stress and it is a sum of the form stress and the subgrid (parameterized frictional) stress in LES. The key non-dimensional parameters to be explored include the wave age $\left(c / u_{* s}\right)$, waveslope $(k a)$, the normalized surface drift velocity $\left(u_{d} / u_{* s}\right)$, and the normalized surface roughness length parameter $\left(k z_{o}\right)$. The first two parameters are approximately matched between the LES and the observations (Table 1).

In observations, a fraction of the wind stress is supported by smaller waves (waves that are not used in the phase-averaging) as well as by the surface viscous stress. In LES, these effects are accounted for by specifying the surface roughness parameterization $z_{o}$ (non-dimensionalized by wavenumber $k$ ) which must be estimated empirically. Following Hara and Sullivan [51], we start with a baseline surface roughness $k z_{o 1}=2.70 \times 10^{-3}$, and reduce it to a half and fourth of this value to align LES results more closely with observed wind speeds and to investi- 
gate the effect of the surface roughness parameterization on the dominant airflow $\left(k z_{o 2}=1.35 \times 10^{-3}\right.$ and $k z_{o 3}=0.67 \times 10^{-3}$, respectively; see Table 1$)$. Therefore, a total of 6 baseline simulations ( 2 wave ages based on observations, 3 surface roughness parameterizations) are performed (Runs 1, 2a, 3a, 4, 5, and 6).

In addition to the baseline simulations, five perturbation simulations are performed with altered surface boundary conditions for the case of $c / u_{* s}=1.4$, including changes to the wave slope $(k a)$, surface drift velocity $\left(u_{d} / u_{* s}\right)$, and alongwave surface roughness $\left(k z_{o}\right)$. The latter two surface boundary perturbations are separated into two simulations each: one with a perturbation to both the crest and trough, and one with a perturbation to the trough only.

By default, LES runs are conducted without a surface drift velocity. Therefore, we add $u_{d} / u_{* s}$ to the surface boundary conditions for two simulations: one with a uniform along-wave drift velocity estimated to be $3 \%$ of the extrapolated $10-\mathrm{m}$ wind speed from observations (i.e. $u_{d} / u_{* s}=0.03 U_{10} / u_{* s}$, run $3 \mathrm{~d}$ ), and one variable (sinusoidal) along-wave drift velocity such that the drift increases toward the trough but disappears towards the crest (i.e. $u_{d} / u_{* s}=a \omega / u_{* s}[1-\cos (k \xi)]$, run $3 \mathrm{~b}$ ). The total horizontal surface velocity (sum of the drift and orbital velocities) becomes constant in this case.

Previous studies have shown that a variable surface roughness parameterization along the wave phase shifts the pressure phase and results in enhanced wave growth that aligns more closely with observations (Gent 43, Gent and Taylor 44, Kudryavtsev and Makin 58). Therefore, we performed two simulations varying the surface roughness $\left(k z_{o}\right)$ along the wave: one in which we increase the roughness by $50 \%$ at the crest and reduce it by $50 \%$ at the trough relative to the constant $k z_{o 3}$ (i.e. $k z_{o 3 n e w}=k z_{o 3}[1+0.5 \cos (k \xi)]$, run $\left.3 \mathrm{e}\right)$, and one in which we reduce roughness 
by $50 \%$ at the trough only (i.e. $k z_{o 3 n e w}=k z_{o 3}[1+0.5 \cos (k \xi)]$ for $\pi / 2<k \xi<3 \pi / 2$, $k z_{\text {o3new }}=k z_{o 3}$ for $k \xi<\pi / 2$ and $3 \pi / 2<k \xi$, run $3 \mathrm{c}$ ). These perturbation runs will illuminate the influence of uniform and along-wave variation of $k z_{o}$ and $u_{d} / u_{* s}$ on the structure of turbulence in the wavy boundary layer in LES.

Finally, another LES is conducted for the intermediate surface roughness $\left(k z_{o 2}\right)$ with a slightly reduced amplitude ( $74 \%$ of the baseline $k a$ ) in order to investigate the response of airflow to modulations in wave steepness under strong wind forcing (Run 2b).

\subsection{Results and discussion}

Note that for the remainder of our analysis, we directly compare the LES results in the $(\xi, \zeta)$ coordinates and the PIV observations in the $\left(\xi^{\prime}, \zeta^{\prime}\right)$ coordinates. We drop the primes from the definition of the PIV mapping.

\section{Results in physical coordinates}

In Figure 1.2, phase-averaged LES and experimental results are presented over the physical wave following $\xi$ - $z$ space for the case of $c / u_{* s}=1.4$. Note that the PIV is plotted as a function of $k \xi$ that is determined from phase detection using Hilbert transform. Snapshots of instantaneous waves appeared sharp-crested with flatter troughs, taking on a surface elevation asymmetry with respect to the mean water surface. While the elevation was asymmetrical, so too was the wave phase with respect to $x$; therefore, the phase-averaged wave shape in observations appeared very close to sinusoidal - that is, the phase detected by the Hilbert transform appeared to stretch/shrink the wave shape horizontally to make it more sinusoidal. Higher frequency wave modes (ripples) were also observed instantaneously in the laboratory 
waves, but none were phase-locked so as to appear in the phase-averaged wave shape. Thus, carrying out the LES and plotting the PIV results over a sinusoidal shape are both suitable for this comparison. The fact that the phase-averaging process using the Hilbert transform almost eliminates the asymmetry (nonlinearity) of the true observed wave shape is one potential reason for some of the discrepancy between the LES and the observation as discussed below.

Wind and waves are directed from left to right, and the phase speed $c$ has been subtracted from the horizontal velocities. Panels 1, 2a, and 3a in Figure 1.2 show LES results for the three baseline surface roughness parameterizations in order of decreasing roughness $\left(k z_{o 1}, k z_{o 2}\right.$, and $k z_{o 3}$, respectively). Panel $2 \mathrm{~b}$ shows the results for $k z_{o 2}$ with wave steepness reduced to $74 \%$ of its baseline $(k a=0.20)$. Panel $3 \mathrm{~b}$ shows the results for $k z_{o 3}$ with a variable surface drift velocity $u_{d} / u_{* s}$ that is zero at the crest and increases toward the trough. Panel 3c shows the results for a variable surface roughness that is equal to $k z_{o 3}$ near the crest and reduces by $50 \%$ at the trough. Panel PIV at the bottom shows corresponding observational results. The turbulent dissipation rate and pressure fields cannot be directly measured from these data, so they are not shown.

The leftmost column shows horizontal wind velocity. While it is not clearly visible in these flow fields, the wind velocity is always negative very close to the surface (moves from right to left) because the results are presented in a coordinate system moving at the wave phase speed; at the interface, the wind speed is equal to $-c$ plus the horizontal wave orbital velocity. A region of reduced horizontal velocity appears on the leeward side of the wave crest in all cases. When flow separates, it creates a large recirculation pattern (bubble) with a weak negative velocity in its lower half. As observed by Buckley and Veron [12] and simulated by Sullivan et al. 
[90], these reduced horizontal velocity patterns in the mean (phase-averaged) flow are associated with transient and intermittent airflow separation events that occur with different frequencies depending on the experimental conditions. No persistent separation events are observed in the instantaneous LES or PIV results.

In the LES with higher surface roughness (panels 1 and 2a), airflow separation events occur so frequently that even the mean flow shows a region of negative velocity. As the roughness is reduced in LES (from panels 1 to $2 \mathrm{a}$ to $3 \mathrm{a}$ ), the signature of airflow separation diminishes but does not disappear, leaving behind the appearance of sheltered but not fully separated flow. This indicates that instantaneous separation events become less frequent and less intense as surface roughness is reduced. The reduction of airflow separation due to reduced background surface roughness is qualitatively consistent with the observation by Gong et al. [45] over a fixed sinusoidal topography.

Although they remain positive, the PIV velocities are dramatically reduced on the leeward side of the wave crest, making the general character and wind speed magnitude of the mean flow similar to LES particularly for the cases of $k z_{o 3}$ (panels 3a-3c). No obvious recirculation bubble is visible in PIV, but a sheltered region of reduced velocity can be observed on the leeward face of the crest, hugging close to the surface in a very thin layer upstream of the equivalent region in LES.

Although 3D TKE fields are available from LES results, PIV only measures the $x$ - and $z$-components of velocity; thus, the two-dimensional TKE (2D TKE) is compared in the second column. The 2D TKE of LES includes two-thirds of the subgrid contribution, assuming isotropic turbulence in the subgrid-scale. In both LES and PIV, the TKE is enhanced downstream of the crest away from the surface, and reduced in a thin layer close to the surface on its lee side. Enhanced 
TKE past the crest and away from the surface indicates advection of turbulent energy downstream by flow that has detached (or separated) from the surface. The advection of TKE may be influenced by the length of the detached free shear layer before it loses coherence downstream. In PIV, the enhanced TKE begins and ends farther upstream and closer to the surface than LES, which suggests that turbulent energy is not advected as far downstream or away from the surface by the separated flow. These results reveal differences between LES and PIV in the behavior of airflow separation - particularly the character of shear layer detachment, sheltering, and reattachment of flow at the following crest. While reducing $k z_{o}$ in LES may improve the comparison of the mean wind speed, the character of phase-averaged shear layer detachment and sheltering still differs considerably from the laboratory observation.

The differences between LES and PIV could be caused by instantaneous changes to the location (phase) and angle of flow separation (detachment) and reattachment, both of which are observed to vary significantly from wave to wave in the tank. These variations may derive from a fluctuating, non-linear laboratory wave shape that deviates from sinusoidal; an asymmetric wave shape can lead to asymmetry in wave orbital velocities, leading to a phase-dependent surface drift velocity. Additionally, at wave ages of $c / u_{* s}=2.5$ and younger, Buckley and Veron [12] observed steepening and occasional breaking with air-entrainment and bubbleproduction. Roughness elements of $O(1 \mathrm{~cm})$ (ripples) were visible extending above the viscous sublayer. These transient processes likely introduced modulations to the dominant wave slope $(k a)$, phase-dependent surface roughness $\left(k z_{o}\right)$, and surface drift velocity $\left(u_{d} / u_{* s}\right)$. These effects are an expected consequence of a wind forcing regime in which very young waves transition from incipient to active breaking. 
The existence of roughness elements and transient surface elevation modulations, especially at the crest, could explain the difference in the character of shear layer detachment observed in PIV. The contours of both TKE and horizontal velocity from PIV suggest that airflow may detach at a much steeper angle as compared to the LES runs with similar wind speed magnitude (panels 3a-3c). The airflow in PIV overshoots the height of the crest and produces a broad fan of reduced velocity downstream with little coherence. The cases of LES with higher surface roughness (panels 1 and 2a) also appear to detach at steeper angles that somewhat resemble PIV, but the downstream character of airflow is disparate. From these results, it appears LES is challenged to thoroughly reproduce the character of airflow in the observation using a steady, sinusoidal wave shape. Under this degree of wind forcing, it may be necessary to account for transient modulations to the spectral character of the waves, including phase-dependent parameters.

We suspected that the difference between the observation and the LES was partially caused by the enhanced/reduced surface roughness (small scale waves) near the crest/trough of the observed waves, since the LES results with uniform along-wave surface roughness all underestimated the wind speed near the trough. We also suspected that the surface drift velocity might vary with phase and modify the flow field above. Thus, the effect of an along-wave variable surface roughness or an along-wave variable surface drift was addressed in a few perturbation runs for the case of $k z_{o 3}$ (Runs $3 \mathrm{~b}, 3 \mathrm{c}$, and 3e). From these runs, we have found that the flow field is mostly determined by the surface conditions (namely, the surface velocity and surface roughness length) near the crest, and that it is insensitive to the surface conditions near the trough. If the surface conditions are altered near the trough only (as in Runs 3b and 3c), the flow field hardly changes. 
While we have anticipated the sensitivity of the flow to the crest conditions, the almost complete insensitivity to the trough conditions is surprising. To demonstrate the second point, we first show the case in which the roughness length is unchanged at the crest but reduced by $50 \%$ at the trough only (Run 3c; Figure 1.2, Panel 3c). The result shows almost no difference from the baseline $k z_{o 3}$ case that shares the same roughness value at the crest but has double the roughness imposed at the trough. We have also performed another case in which the roughness is enhanced/reduced by $50 \%$ at the crest/trough (Run 3e, not shown), and have found that the flow field is not distinct from what we would expect if we imposed a $50 \%$ enhanced $k z_{o}$ that was uniform along the wave.

A similar pattern occurs with the addition of surface drift velocity. A surface drift added only at the trough (Run 3b; Figure 1.2, Panel 3b) hardly affects the flow field at all compared to the baseline $k z_{o 3}$ case. A uniform (phase-independent) surface drift (Run 3d; not shown) alters the flow field by simply increasing the phase averaged wind speed uniformly, but not affecting the wind shear or turbulence characteristics.

In summary, the surface boundary conditions near the crest (namely, horizontal surface velocity and surface roughness) appear to serve the foremost importance in the structure of airflow in LES, while conditions near the trough are of little importance. This finding also suggests that if the wave phase speed or orbital velocities are slightly modified (e.g., by accounting for the finite amplitude effect), the phase averaged wind velocity will uniformly increase/decrease by the increase/decrease of total velocity at the crest (horizontal velocity + drift velocity - phase speed), but the other flow quantities will be hardly affected.

The turbulent dissipation rate in LES (third column) shows enhanced dissipation 
of TKE at the trailing edge of the crest where wind shear is strongest. In all six LES cases shown, enhanced dissipation is advected downstream by the detached flow, but it is most apparent in the cases with higher surface roughness (panels 1 and 2a). This is compatible with the advected 2D TKE shown in the second column. In the LES cases with reduced $k z_{o}$ and $k a$, the region of enhanced TKE and dissipation rate hugs closer to the surface, altering the airflow that presumably reattaches on the windward face of the following wave at a stagnation (or "splat") point. There is a small region of reduced dissipation where similarly reduced TKE and horizontal velocity are present on the lee side of the wave, as observed by Hara and Sullivan [51]. This sheltered region (or "dead zone") moves downstream and shrinks with reduced $k z_{o}$ and $k a$, most notably for the case with reduced $k a$. The splat point also changes with reduced $k z_{o}$ and $k a$, which is capable of modulating the pressure field over the wave (discussed next).

Pressure fields are shown in the fourth column of Figure 1.2. The peak pressure is typically located on the windward face of the wave, near where flow reattaches at the splat point. As $k z_{o}$ is reduced, the splat point shifts upstream and the surface pressure is visibly enhanced. This effect is likely influenced by the character of flow separation that modifies the downstream airflow, including the force with which it strikes the surface. Strong separation upstream leads to turbulent flow carried farther downstream and dissipated, causing the splat to occur farther along the following wave with reduced force. This explains why lower $k z_{o}$ (which experiences less intense flow separation) produces a splat farther upstream towards the trough with more intense pressure.

The pressure fields for cases of $k z_{03}$ show more drastic along-wave pressure gradients. Reduced roughness at the crest allows the airflow to accelerate there, 
reducing the pressure and preventing strong flow separation or sheltering that reduces the mean wind speed. Again, these flow characteristics are primarily driven by surface boundary conditions near the crest where flow separation is initiated, and not at the trough. In the cases of enhanced surface drift velocity and reduced roughness at the trough only (panels $3 \mathrm{~b}$ and 3c, respectively), the nature of airflow separation in the physical flow fields does not visibly change from the baseline $k z_{o 3}$ (panel 3a). This again suggests that the sluggish airflow behind the wave during separation or sheltering diminishes the impact of the surface boundary conditions at that location.

Alterations to the pressure field by airflow separation modulate the phasedependent surface pressure that determines the overall form drag. The case of reduced $k a$ begins to show distinctions from reduced $k z_{o}$ here, experiencing an upstream shift in the phase of peak pressure similar to the $k z_{o 3}$ but without the drastic along-wave pressure gradients. This result is a sign of a lower propensity for flow separation to occur over waves with a lower $k a$. The results for the case of reduced $k a$ are qualitatively consistent with the results of Donelan et al. [27], who used in-situ wave-following observations to analyze the correlation between wave slope and the occurrence of flow separation. They suggest a physical mechanism for flow separation whereby sharpening and steepening of the wave crest reduces the wave-induced vertical pressure gradient that maintains the centripetal acceleration necessary to keep streamlines attached to the surface. This causes flow to separate and ultimately the wave to spill over and break. While our LES did not experiment with the curvature at the wave crest, we altered the wave slope for run $2 \mathrm{~b}$ and found that its effects alone may play a significant role in modifying the frequency and strength of air flow separation. 


\section{Results in mapped coordinates}

The wave-following mapping utilized in Hara and Sullivan [51] is used to present the wind turbulence characteristics close to the surface. This mapping allows for an assessment of the effects of the wave shape at constant normalized heights above the waves, $k \zeta$. Figure 1.3 shows the mean normalized horizontal wind speed minus the wave phase speed, $(\langle u\rangle-c) / u_{* s}$ for both wave ages (column a), accompanied

by the associated mean normalized wind shear (column b), $\left(\frac{\partial<u>}{\partial \zeta}\right)\left(\frac{\kappa \zeta}{u_{* s}}\right)$, and mean normalized 2D TKE (column c), $\left(\left\langle u^{\prime} u^{\prime}\right\rangle+\left\langle w^{\prime} w^{\prime}\right\rangle\right) / u_{* s}^{2}$.

Baseline LES runs are shown in dark blue with solid, dashed, and dotted lines characterizing $k z_{o}$ in order of decreasing roughness $\left(k z_{o 1}, k z_{o 2}\right.$, and $k z_{o 3}$, respectively). This line characterization is repeated for the perturbation runs using different colors: the dashed green line refers to $k z_{o 2}$ with reduced $k a$, and orange and purple dotted lines refer to the phase-dependent $u_{d} / u_{* s}$ and $k z_{o 3}$ runs, respectively. PIV results are shown as solid black lines. The light grey line in column b is the profile of the unperturbed wind shear experienced by the pressure-driven channel flow in both LES (solid) and PIV (dotted). As can be seen, the fitted wind shear profile in PIV is almost identical to LES.

All results are plotted in log scale in the vertical to focus on the effects very close to the surface. Below $k \zeta \approx 0.025$, results are omitted because the effects of unresolved viscosity and small-scale waves in LES become dominant. Above $k \zeta \approx 0.824$, PIV results are omitted due to noise. Above about $k \zeta=0.7$, the shape of the mean wind profile in LES returns to an almost straight line in log coordinates, indicating that at this location the wind approaches the typical logarithmic profile expected over a flat surface, slightly modified due to the slowly decreasing wind 
stress with increasing height above the water surface. Here, the LES differs from PIV in that the latter is still varying with height at the highest point of observation.

For both wind forcing cases, the magnitude of the mean wind in observations compares well with the cases of LES with $k z_{o 3}$. Below about $k \zeta=0.7$, the LES profiles deviate from the characteristic log profile for a flat surface from MoninObukhov similarity theory, indicating that the waves are altering the structure of the mean wind; this in turn will affect the equivalent roughness length $z_{o, e}$ (i.e. the parameter that sets the bulk surface roughness in the typical logarithmic wind profile). This wave-modulated deviation can be more readily seen in the profile of normalized mean wind shear (Figure 1.3, panel b), which shows regions of enhanced (reduced) wind shear to the right (left) of the unperturbed wind shear without waves (light grey line), respectively. The enhanced wind shear in this plot is indicative of the onset of airflow separation events that enhance the turbulence at a height comparable to the wave amplitude (see Figure 1.2). Closer to the surface, wind shear is reduced to a value less than that expected over a flat surface. As Hara and Sullivan [51] noted, the resulting $z_{o, e}$ is affected by the reduction of wind shear close to the surface, where momentum flux is dominated by wave-induced (pressure) stress. The magnitude and height of the enhanced wind shear are reduced as $k z_{o}$ is decreased, consistent with the physical LES velocity fields (Figure 1.2). For the case with a reduced wave slope $k a$, the enhanced wind shear is shifted closer to the surface as expected, but does not reduce magnitude as it does for cases with $k z_{o 3}$; in fact, the wind shear is slightly enhanced above its baseline $k z_{o 2}$ in this case. Figure 1.2 sheds some light on the reason for this difference. The case of reduced $k a$ appears to separate somewhat differently than the cases of reduced $k z_{o 3}$; while streamlines stay tight to the surface in the former, the latter fans out more 
significantly downstream, producing slightly less TKE over the crest, but more above the trough (see Figure 1.2, column 2). This effect is only slightly noticeable in the profiles of mean wind speed.

The PIV results also show enhanced wind shear near the crest level and reduced wind shear further below. While wind shear from PIV is strongly reduced towards the surface as expected, enhanced wind shear is not nearly as prominent compared to LES. This effect is not entirely surprising considering the appearance of the physical flow fields (Figure 1.2) suggest that the laboratory waves experience modulations to the wave parameters that impact the character of wave-produced turbulence. For example, the wave slope varies from wave to wave in the observations as waves intermittently break; this could enhance wind shear at different heights from wave to wave, smearing the mean wind shear profile. The LES case with reduced $k a$ exemplifies the potential effect wave slope modulations have on the wind shear. Nevertheless, the mildly enhanced wind shear from PIV is at a comparable height to LES, suggesting that the laboratory waves most often experience enhanced turbulence from instantaneous flow separation at this height. The mean 2D TKE (Figure 1.3, Panel c) is enhanced at the same height for both LES and PIV, but the latter stays high closer to the surface. This is consistent with the results in Figure 1.2. For all of our simulations, the subgrid contribution steadily increases towards the surface, but none reach a subgrid contribution over $60 \%$ of the total shown at the lowest point of the 2D TKE profile. Thus, we believe the differences between LES and PIV are not a result of the subgrid closure, but rather the wave perturbations in the tank not captured in the phase-averaged wind fields. 


\section{Momentum flux budget}

The momentum flux budget is derived from a triple decomposition of wind velocity into mean, turbulent, and wave-coherent components. This allows for a partition of the total momentum flux (or wind stress) into three contributions to describe how the waves alter the transfer of horizontal momentum across constant $\zeta$ surfaces: turbulent stress, wave-coherent stress, and pressure stress. The triple decomposition and derivation of the momentum and energy equations in wavefollowing coordinates for this study are described in more detail in Hara and Sullivan [51], and have been used in past studies to describe the momentum flux partition in the presence of waves (Chalikov and Rainchik 17, Sullivan et al. 92). The turbulent (or Reynolds) stress, $\tau^{t}$, is defined as the average of the product of turbulent fluctuation velocities, $\left\langle u^{\prime} W^{\prime}\right\rangle$, and includes the subgrid-scale contribution. The wave-coherent stress, $\tau^{w}$, is defined as the average of the product of wave-coherent

velocities, $\langle\tilde{u} \tilde{W}\rangle$, which arises when the phase-averaged wind velocity crosses the constant $\zeta$ surfaces. The pressure stress, $\tau^{p}$, results from the pressure exerted on the tilted constant $\zeta$ surface, and is primarily responsible for wave growth at the surface.

Because wind is driven by a horizontal pressure gradient in LES and the laboratory flume, the total wind stress varies linearly and the full $x$-momentum equation includes the three stress contributions as well as the vertically integrated pressure gradient as in (1.7). The vertical profiles of the four terms on the left of (1.7) are plotted for LES and observations under both experimental wind-forcing conditions in Figure $1.4\left(c / u_{* s}=1.6\right.$ and 1.4 for top and bottom panels, respectively). The results for wave-coherent stress, pressure stress, turbulent stress, and total 
wind stress including the pressure gradient are shown in columns a, b, c, and d, respectively. The line colors and styles correspond identically with Figure 1.3, except for the viscous stress from PIV (column a; solid pink line). The pressure stress from PIV (panel b) is dot-dashed to signify that it has been calculated as the residual of the total wind stress after turbulent, wave-coherent, and viscous stress contributions have been removed; this is because pressure stress cannot be measured directly from the observations. The vertically integrated pressure gradient and the linearly decreasing wind stress which balances it - shown by grey lines for both LES (solid) and PIV (dashed) - are shown in panels b and c, respectively. Notice that both of these profiles turn out to be almost identical between the LES and the PIV for the case of $c / u_{* s}=1.4$.

It is important to note that sea spray has not been considered in this analysis, although it has been observed frequently over strongly forced conditions such as these, and has been suggested as a potential explanation for the reduction of the drag coefficient under tropical cyclone wind forcing (Donelan 26, Powell et al. 82). Previous studies have assessed the impact of sea spray on air-sea momentum and energy fluxes (Fairall et al. 37, Richter and Sullivan 85, Veron 102), and LES has proven to be a promising method for this type of investigation. Nevertheless, we have focused solely on the wave parameters in this study and will leave the effect of sea spray for a future study.

Both wind forcing cases $\left(c / u_{* s}=1.6\right.$ and 1.4) show similarities between LES and PIV in the horizontal mean partition of momentum flux (Figure 1.4), especially in low roughness $\left(k z_{o 3}\right)$. Near the crest height, the mean normalized wave-coherent stress $\left(<\tau^{w}>/ \tau_{s}\right.$; column a) is negative. Since each stress component is normalized by $\tau_{s}$ which is negative (indicating flux downwards), a negative value of normalized 
wave-coherent stress is associated with upward momentum flux. This effect is the result of the wave-following coordinate system that sees accelerated mean flow that detaches (separates) away from the wavy surface towards a more positive constant- $\zeta$ surface - that is, $\tilde{u}$ is positive (larger than the mean) and $\tilde{W}$ is positive (moving away from the surface) downwind of the peak of the wave crest, so the normalized wave-coherent stress is negative. When flow reattaches upwind of the following crest, $\tilde{u}$ is negative (smaller than the mean) and $\tilde{W}$ is also negative (moving towards the surface), so the normalized wave-coherent stress is again negative.

The spatial pattern of the phase-averaged wave-coherent stress can be seen in Figure 1.5 (column 1), where regions of negative normalized wave-coherent stress (upward flux) occur approximately at separation and reattachment points (0 to $\pi / 2$ and $\pi$ to $3 \pi / 4$ ), and are much more intense than the positive (downward flux) regions where flow is more sluggish. This pattern is what causes a horizontal mean wave-coherent stress that is increasingly negative (upward flux) with enhanced airflow separation events and sheltering. In PIV, the wave-coherent stress is intensely negative at the approximate location of airflow separation but is not significant near the reattachment point, which supports our earlier suggestion that the laboratory waves experience some differences from LES in the character of flow separation and reattachment. For the case of the lowest roughness $\left(k z_{o 3}\right)$ in LES, wave-coherent stress is less intense at the point of separation, while for the case of highest roughness $\left(k z_{o 1}\right)$ the stress at this location matches the magnitude of PIV more closely. Regardless, the wave-coherent stress in PIV averaged over the entire wave compares reasonably with the LES results, most closely with the $k z_{o 3}$ cases (Figure 1.4).

Negative wave-coherent stress near the crest height is compensated by the 
enhancement of turbulent stress $\left\langle\tau^{t}\right\rangle / \tau_{s}$ (Figure 1.4, column c) exceeding the total wind stress, which is correlated with enhanced mean wind shear near the peak height of the crest, as discussed by Hara and Sullivan [51]. The reduction of turbulent stress that can be seen close to the surface for both $c / u_{* s}=1.6$ and 1.4 is associated with increasing pressure stress, which is always positive for both LES and PIV for strongly-forced waves (downward flux), as expected. This is correlated with the reduced mean wind shear towards the surface. For the case of $c / u_{* s}=$ 1.4 (Figure 1.4, bottom panel), the mean turbulent stress for the LES cases of $k z_{o 3}$ compare remarkably well with PIV above $k \zeta \approx 10^{-1}$. Below this level LES and PIV begin to diverge for both $c / u_{* s}=1.6$ and 1.4 , with PIV experiencing a more rapid decrease in turbulent stress towards the surface. This divergence also causes diverging pressure stress contributions between the LES and PIV because the pressure stress is calculated as the residual of the total stress in PIV.

One possible reason for this divergence is that the LES turbulent stress is influenced by the subgrid (SGS) parameterization. In fact, below $k \zeta \approx 10^{-1}$, the SGS stress begins to dominate the total turbulent stress. However, we believe that the pressure stress component should remain relatively uniform with height near the wave surface because the pressure field itself is expected vary slowly with height (from the thin boundary layer approximation). It is more likely that the observed turbulent stress becomes less accurate very near the surface because of strongly fluctuating laboratory waves superimposed by smaller scale waves.

\section{Surface stresses and wave growth rate}

Using the LES results, we next investigate how the surface stresses (pressure, normal turbulent stress, tangential turbulent stress) and the wave growth rate are 
modified by varying $k z_{o}, k a$, and $u_{d} / u_{* s}$. The along-wave surface tangential stress is obtained by phase averaging the instantaneous surface tangential stress, which is calculated from the LES instantaneous tangential velocity at the first grid level and the specified roughness length $k z_{o}$. In reality, this tangential stress corresponds to a sum of the surface viscous stress and the form drag of smaller unresolved waves. In LES, the pressure and the normal stress are calculated at the first grid level, not at the surface. We have ascertained that a sum of the pressure and the normal stress (total normal stress) is approximately constant within the first few grid levels. Therefore, the true surface pressure is very close to the total normal stress evaluated at the first grid level. These phase-averaged surface stresses are shown in Figure 1.6 for the wind forcing case of $c / u_{* s}=1.4$.

The pressure fields in Figure 1.2 and the pressure stress in Figure 1.4 suggest that the surface pressure distribution is significantly modified by varying wave parameters, which is confirmed in the along-wave surface pressure distribution shown in Figure 1.6. For $k z_{o 1}$, there is a region of very small negative and constant pressure below the dead zone produced by flow separation, roughly between $k x=\pi / 2$ and $\pi$. The peak surface pressure is significantly shifted downstream from the trough; that is, wind is pushing the wave. Reducing from $k z_{o 1}$ to $k z_{o 2}$ and then $k z_{o 3}$ weakens the flow separation effect (smoothing the surface pressure distribution below the "dead zone"), causing the phase of peak pressure to shift upstream slightly and the magnitude of along-wave pressure gradient to increase significantly. As might be expected from our earlier results, the surface pressure distribution for the $k z_{o 3}$ perturbation runs in which surface boundary conditions are only altered in the trough barely change from their baseline $k z_{o 3}$. For the case of reduced $k a$ (dashed green line), the surface pressure distribution is "smoothed" similar to reducing $k z_{o}$, 
but the along-wave pressure gradient does not change drastically. This should be proportional to the wave amplitude according to the linear perturbation theory.

Using the phase-averaged surface stresses, the non-dimensional wave growth rate coefficient $c_{\beta}$ is computed by finding the energy fluxes due to the (total) normal stress $\overline{\tau_{n}}$ and the tangential stress $\overline{\tau_{t}}\left(E F_{n}\right.$ and $E F_{t}$, respectively) into the waves as in Hara and Sullivan [51]:

$$
c_{\beta}=2 \frac{E F}{u_{* s}^{3}} \frac{u_{* s}}{c}(k a)^{-2}
$$

where

$$
\begin{gathered}
E F_{n}=\left\langle\frac{\overline{u_{n} \tau_{n}}}{\cos \theta}\right\rangle, \quad E F_{t}=\left\langle\frac{\overline{u_{t} \tau_{t}}}{\cos \theta}\right\rangle \\
E F=E F_{n}+E F_{t}
\end{gathered}
$$

where $\theta$ is the point-by-point angle of surface tilt from the horizontal, and $\overline{u_{n}}$ and $\overline{u_{t}}$ are normal and tangential components of the wave orbital velocity at the surface, respectively.

The total wave growth coefficient $c_{\beta t o t}$ for each LES condition is listed in Table 2, along with its tangential stress, normal stress, and pressure stress contributions $\left(c_{\beta t}, c_{\beta n}\right.$, and $\left.c_{\beta p}\right)$. As expected, the wave growth is dominated by the pressure form drag (the total normal stress), which can be seen by comparing the upper and lower panels of Figure 1.6. As $k z_{o}$ is reduced, the total wave growth rate mildly decreases. Although the along-wave pressure gradient increases with reduced $k z_{o}$, the phase of the peak pressure shifts toward the trough and reduces the form drag (Figure 1.6). The addition of $u_{d} / u_{* s}$ for runs $3 \mathrm{~b}$ and $3 \mathrm{~d}$ has almost no impact on the total growth rate. The phase dependent $k z_{o}$ varying from baseline in the trough 
only (run 3c) also has little impact on growth rate; that varying from baseline at both the crest and trough (run 3e) approaches the 2a case where the roughness near the crest is the same. The most interesting finding is that the total growth rate significantly increases (by 26\%) as the waveslope $k a$ decreases (by 26\%). This is qualitatively consistent with the earlier finding that the pressure variation does not scale with the wave steepness. This means that the concept of the linear wave growth rate (the wave growth rate being independent of wave slope) does not apply here, which is consistent with the observational results of Peirson and Garcia [79]. Between the two wind forcing conditions (top and bottom in Table 2), the higher wave age $\left(c / u_{* s}=1.6\right.$, bottom) has a slightly higher growth rate (by $5-6 \%$ ), primarily caused by higher pressure contribution (turbulent normal and tangential contributions change very little). The value of $k a$ for this condition is slightly lower (by $4 \%$ ). It is therefore possible that this increase of the growth rate is mainly due to the associated decrease of $k a$ rather than the change in the wave age.

Many operational wave models assume that the wave growth rate is independent of wave slope and is related to the wind stress or the wind speed for a given wavenumber. Our results, however, show that the growth rate is dependent on the wave slope, consistent with the findings of Sullivan et al. [90]. When the surface roughness (effect of unresolved small waves) varies, the growth rate also changes even though the wind stress is held constant, i.e., the growth rate is not determined by the wind stress alone. As the surface roughness decreases, the wind speed increases, but the growth rate decreases. Therefore, the growth rate is not determined by the wind speed alone, but rather is modified by both the wave slope and the surface roughness. 


\section{Wave effect on equivalent surface roughness}

Since the mean wind speed profiles of LES roughly approach those over a flat surface (logarithmic profiles slightly modified by the slowly decreasing wind stress with height) for $k \zeta>0.7$ (Figure 1.3, left panel), the equivalent surface roughness $z_{o, e}$ can be estimated by extrapolating the profiles above $k \zeta>0.7$ towards the surface. The results of $k z_{o, e}$ as well as the ratio $z_{o, e} / z_{o}$ (the enhancement of roughness length by the waves) are summarized in Table 3. Note that the quantity $\log \left(z_{o, e} / z_{o}\right)$ is proportional to the increase of wind speed outside the wave boundary layer due to the wave. Hara and Sullivan [51] discuss how $z_{o, e} / z_{o}$ increases if the normalized mean wind shear is reduced near the water surface, and decreases if the normalized mean wind shear is enhanced due to flow separation, as shown in Figure 1.3 (middle column). The total wave effect on $z_{o, e}$ and the drag coefficient is determined by a sum of these two effects.

As the specified normalized surface roughness $k z_{o}$ decreases, $k z_{o, e}$ naturally decreases as well, but much less so; thus, the ratio $z_{o, e} / z_{o}$ increases significantly. Therefore, the wave effect on increasing surface roughness is more pronounced as the surface roughness decreases. This is consistent with the fact that decreasing surface roughness weakens the flow separation effects and the associated enhancement of mean wind shear. It is interesting that a similar effect was seen in a recent modeling study for turbulence in the oceanic wave boundary layer (Teixeira 97); the equivalent (or effective) surface roughness was obtained by extrapolating the logarithmic current profile and showed enhancement over the imposed value in conditions of wave-dominated turbulence (see their Figure 3).

As the wave slope $k a$ decreases, $z_{o, e} / z_{o}$ significantly decreases as expected. 
Adding constant surface drift velocity $u_{d} / u_{* s}$ seems to shift the wind speed almost uniformly, inside and outside the wave boundary layer, but does not significantly affect $k z_{o, e}$, since this value is found after subtracting $u_{d} / u_{* s}$ from the mean wind speed profile. Since adding a phase-varying $u_{d} / u_{* s}$ that approaches zero towards the crest does not visibly affect the wind speed (as in run 3b), it appears that the presence of $u_{d} / u_{* s}$ at the crest dominates the airflow characteristics, a similar effect to when $k z_{o}$ is imposed at the crest. When constant surface drift is imposed along the wave (Run 3d) the turbulent features in the airflow remain intact, and the only visible difference from the baseline is a small, vertically uniform increase in the wind speed. This suggests that the wave effect on the turbulence is not significantly altered by the surface drift.

\subsection{Summary}

Wind turbulence over strongly-forced wind-waves was simulated using LES and was compared with laboratory PIV observations for wave ages of $c / u_{* s}=1.4$ and 1.6 (very young waves) and respective wave slopes of $k a=0.27$ and $k a=0.26$. LES was performed over a periodic sinusoidal wave train and the results were phaseaveraged. Since the laboratory waves were solely wind-generated, they were not strictly periodic, and hence the Hilbert transform of the wave elevation record was used to determine the wave phase before phase-averaging of the results. Although the laboratory waves experienced frequent modulations to the wave slope, wave shape, and visible surface roughness, the phase-averaged laboratory wave shape appeared remarkably sinusoidal. Therefore we have used a sinusoidal wave shape for the phase-averaged LES comparison. Three different values of constant surface

roughness (representing the effect of the surface viscous stress and the form drag 
of unresolved small waves) were used to match the wind speed in LES with the observation $\left(k z_{o 1}, k z_{o 2}\right.$, and $\left.k z_{o 3}\right)$.

The results show a reasonable comparison of LES and PIV, especially for the case of $k z_{o 3}$ conditions in LES. Both results show the signature of instantaneous airflow separation events (or sheltering), enhancement of the horizontally averaged wind shear profile near the crest, and reduction towards the surface (Figure 1.3). They also show similar patterns of wave-coherent and turbulent stress which result in a good agreement of the momentum flux budget, particularly for the LES case of $k z_{03}$ (Figures 1.4 and 1.5). However, important differences are observed in the mean flow patterns and the spatial distribution of 2D TKE, particularly near the crest and downstream. The disparity in the magnitude and location of turbulent features may have resulted from a number of differences between LES and the laboratory set-up. These include the spectral spread, transient modulations, smaller scale waves, non-linearity of the wind-generated laboratory waves compared with the periodic monochromatic waves modeled in LES, all of which can influence phase determination and averaging. The results suggest that wave characteristics at the crest play a key role in determining how the flow detaches/reattaches on the leeward/windward face of the wave when it is strongly forced, as has been found by previous studies such as Donelan et al. [27] mentioned earlier. Airflow over laboratory waves appears to detach at a steeper angle than LES while at the same time hugging closer to the surface and showing a somewhat incoherent detached free shear layer. LES exhibits a more coherent signature of airflow separation, suggesting that instantaneous airflow separation events are more consistently advecting turbulence away from the surface.

To address these disparities, five LES perturbation runs were performed: one 
with a $26 \%$ reduced wave slope $k a$ (run $2 \mathrm{~b}$ ), two with constant and phase-varying surface drift velocity (runs $3 \mathrm{~d}$ and $3 \mathrm{~b}$ ), and two with phase-varying surface roughness (runs $3 \mathrm{c}$ and $3 \mathrm{e}$ ). Airflow in the runs with phase-varying surface boundary conditions showed no sensitivity to modifications near the trough, indicating that the conditions near the crest play a primary role in the turbulent flow over strongly forced waves. The LES results also show strong sensitivity to the wave slope $k a$ while maintaining the same wind forcing. Decreasing $k a$ lowers the height of enhanced mean wind shear and the signature of airflow separation events in general, which could explain disparities between LES and PIV if laboratory waves are experiencing frequent amplitude modulations. While decreasing $k z_{o}$ mildly increases the equivalent roughness length $\left(z_{o e} / z_{o}\right)$ and decreases the wave growth rate, decreasing $k a$ reduces $z_{o e} / z_{o}$ and significantly increases the wave growth rate (Tables 2 and 3), in agreement with Peirson and Garcia [79] and Sullivan et al. [90].

Overall, the results presented here demonstrate reasonable fidelity of LES to represent wind over surface waves in strongly forced conditions in a wind wave flume, particularly for horizontally averaged quantities, and illuminate the effect of surface waves on the mean wind profile and equivalent surface roughness (drag coefficient). Namely, near the height of the wave amplitude, transient flow separation causes upward wave-coherent stress, enhanced downward turbulent stress, and enhanced mean wind shear. Further below, the downward pressure stress is balanced by the reduced downward turbulent stress and reduces the mean wind shear. These findings are consistent with those by Hara and Sullivan [51], and can be used to develop improved parameterizations of sea-state dependent air-sea momentum flux (drag coefficient).

The results also raise questions as to the surface boundary conditions needed to 
adequately represent wave fluctuations and spectral spread in a wind-wave regime of this nature. The present results suggest that the effects of wave characteristics specifically near the crest should be explored further. More work is required to understand the disparities in the phase-averaged character of instantaneous airflow separation events under strongly forced conditions, including the location, intensity, and angle of detachment and reattachment of airflow at the surface. It would also be desirable to use LES to further explore the effects of sea spray (Richter and Sullivan 85), unsteady waves (Sullivan et al. 90), and fully-coupled wind and waves (Chalikov and Rainchik 17) in gaining a more thorough understanding of a strongly forced wind-wave regime on air-sea momentum flux and wave growth rate. Additionally, we would like to extend the present LES study to explore airflow characteristics of conditions that are difficult to achieve in laboratory observations, including misaligned wind-wave conditions, more mature wave ages, and the effects of surface waves on mean temperature, humidity, and scalars, including their transfer coefficients.

\subsection{Acknowledgements}

We acknowledge support of the National Science Foundation (Physical Oceanography) Grants OCE-1458984 (URI), OCE-1458977 (UD) and OCE-1634051 (UD). We also acknowledge high-performance computing support from Yellowstone and Cheyenne (doi:10.5065/D6RX99HX) provided by NCAR's Computational and Information Systems Laboratory, sponsored by the National Science Foundation.

MB acknowledges support from the National Science Foundation Atmospheric

and Geospace Sciences Postdoctoral Research Fellowship AGS-PRF-1524733, and from the Deutsche Forschungsgemeinschaft (DFG, German Research Foundation, 
project number 274762653, Collaborative Research Centre TRR 181 ("Energy Transfers in Atmosphere and Ocean"). 


\subsection{Figures and Tables}

Table 1.1: Non-dimensional parameters used in eleven LES experiments: wave age $\left(c / u_{* s}\right)$, wave steepness $(k a)$, surface roughness $\left(k z_{o}\right)$, and surface drift velocity $\left(u_{d} / u_{* s}\right)$. In the text and figure captions, surface roughness $k z_{o}=2.70 \times 10^{-3}, 1.35 \times$ $10^{-3}$, and $0.67 \times 10^{-3}$ are referred to as $k z_{o 1}, k z_{o 2}$, and $k z_{o 3}$, respectively. For Run $3 \mathrm{c}, k z_{\text {o3new }}=k z_{03}$ from $k x=\frac{\pi}{2}$ to $3 \frac{\pi}{2}$.

\begin{tabular}{|c|c|c|c|c|}
\hline Run & $c / u_{* s}$ & $k a$ & $k z_{o}\left(\times 10^{-3}\right)$ & Perturbation \\
\hline 1 & 1.4 & 0.27 & 2.70 & - \\
\hline $2 \mathrm{a}$ & 1.4 & 0.27 & 1.35 & - \\
\hline $2 \mathrm{~b}$ & 1.4 & 0.20 & 1.35 & $k a_{n e w}=0.74 k a$ \\
\hline $3 a$ & 1.4 & 0.27 & 0.67 & - \\
\hline $3 b$ & 1.4 & 0.27 & 0.67 & $\frac{u_{d}}{u_{* s}}=-\frac{a \omega}{u_{* s}}[\cos (k x)-1]$ \\
\hline $3 c$ & 1.4 & 0.27 & 0.67 & $k z_{o 3 n e w}=k z_{o 3}[1+0.5 \cos (k x)]\left(k x=\frac{3 \pi}{2}\right.$ to $\left.\frac{\pi}{2}\right)$ \\
\hline $3 \mathrm{~d}$ & 1.4 & 0.27 & 0.67 & $\frac{u_{d}}{u_{* s}}=\frac{0.03}{u_{* s}} U_{10}$ \\
\hline $3 \mathrm{e}$ & 1.4 & 0.27 & 0.67 & $k z_{\text {o3new }}=k z_{03}[1+0.5 \cos (k x)](k x=0$ to $2 \pi)$ \\
\hline 4 & 1.6 & 0.26 & 2.70 & - \\
\hline 5 & 1.6 & 0.26 & 1.35 & - \\
\hline 6 & 1.6 & 0.26 & 0.67 & - \\
\hline
\end{tabular}


Table 1.2: Values of non-dimensional wave growth coefficient $c_{\beta}$ for both wind forcing cases $\left(c / u_{* s}=1.4\right.$ on top; $c / u_{* s}=1.6$ on bottom). The values for $c_{\beta t o t}, c_{\beta t}, c_{\beta n}$, and $c_{\beta p}$ refer to total, tangential stress, normal stress, and pressure contributions of $c_{\beta}$. For references to runs, refer to Table 1 .

$\begin{array}{ccccccccc}\text { Run } & 1 & 2 a & 2 b & 3 a & 3 b & 3 c & 3 d & 3 e \\ c_{\beta t o t} & 17.5 & 15.9 & 20.1 & 14.1 & 14.6 & 14.4 & 14.1 & 15.4 \\ c_{\beta t} & 2.7 & 2.6 & 3.8 & 2.6 & 2.7 & 2.6 & 3.2 & 2.2 \\ c_{\beta n} & 1.6 & 1.0 & 0.9 & 0.3 & 0.2 & 0.3 & 0.1 & 0.5 \\ c_{\beta p} & 13.2 & 12.4 & 15.4 & 11.2 & 11.7 & 11.5 & 10.8 & 12.8 \\ \text { Run } & 4 & 5 & & 6 & & & & \\ c_{\beta t o t} & 18.3 & 16.9 & - & 14.9 & - & - & - & - \\ c_{\beta t} & 2.8 & 2.7 & - & 2.6 & - & - & - & - \\ c_{\beta n} & 1.7 & 1.1 & - & 0.4 & - & - & - & - \\ c_{\beta p} & 13.9 & 13.2 & - & 11.8 & - & - & - & -\end{array}$

Table 1.3: Values of normalized equivalent surface roughness $k z_{o e}$ for both wind forcing cases $\left(c / u_{* s}=1.4\right.$ on top; $c / u_{* s}=1.6$ on bottom). The value of $z_{o, e} / z_{o}$ is the ratio of the equivalent surface roughness $z_{o, e}$ to the parameterized surface roughness $z_{o}$. For references to runs, refer to Table 1 .

$\begin{array}{ccccccccc}\text { Run } & 1 & 2 a & 2 b & 3 a & 3 b & 3 c & 3 d & 3 e \\ k z_{o, e} \times 10^{-3} & 11.51 & 7.90 & 3.55 & 4.53 & 4.10 & 4.57 & 4.41 & 5.47 \\ z_{o, e} / z_{o} & 4.26 & 5.85 & 2.63 & 6.77 & 6.12 & 6.82 & 6.59 & 8.16 \\ \text { Run } & 4 & 5 & & 6 & & & & \\ k z_{o, e} \times 10^{-3} & 10.74 & 7.24 & - & 4.24 & - & - & - & - \\ z_{o, e} / z_{o} & 3.98 & 5.36 & - & 6.32 & - & - & - & -\end{array}$




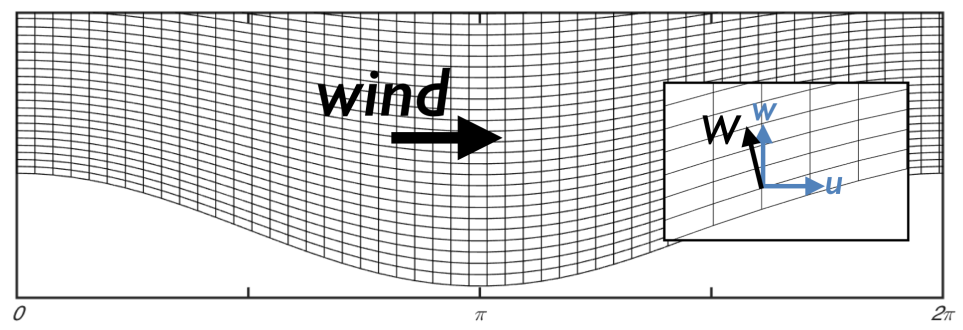

Figure 1.1: Schematic of the wave-following mapping used for analysis of both LES and PIV results introduces a vertical coordinate $\zeta$ that follows the wave shape at $\zeta=0$ and gradually approaches the Cartesian $z$ coordinate as $\zeta$ increases. This mapping enables analyses below the wave crest level. The vertical momentum flux is defined using a Cartesian velocity $u$ and a contravariant velocity $W$ as shown in the small box. 

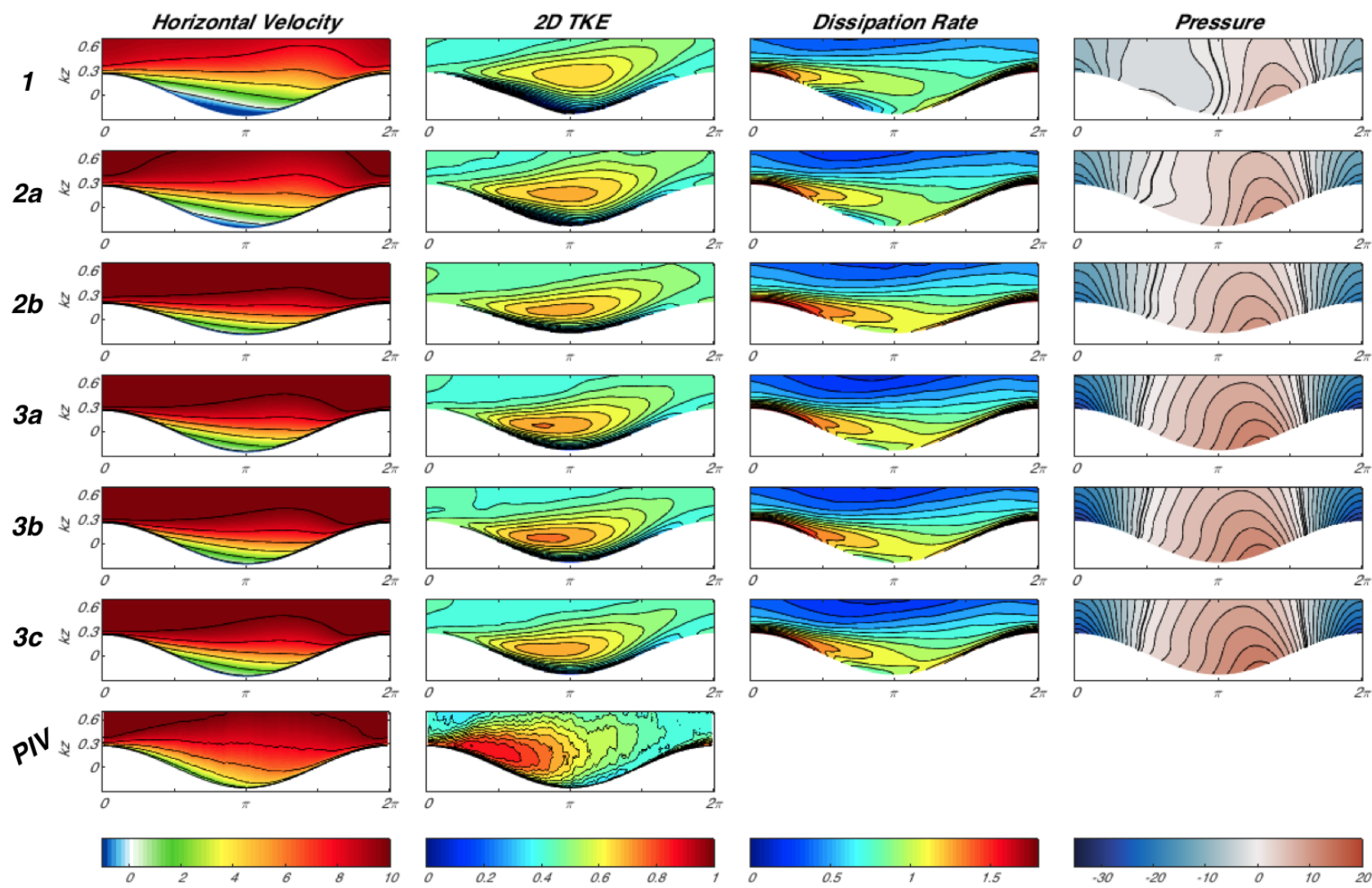

Figure 1.2: Phase-averaged flow fields from left to right: horizontal velocity, twodimensional turbulent kinetic energy, dissipation rate, and pressure. Each panel is labeled corresponding to its run number (see Table 1). Observations are shown on the bottom panel. All fields are non-dimensionalized using surface friction velocity $\left(u_{* s}\right)$, surface stress $\left(\tau_{s}\right)$ and wavenumber $(k)$. 

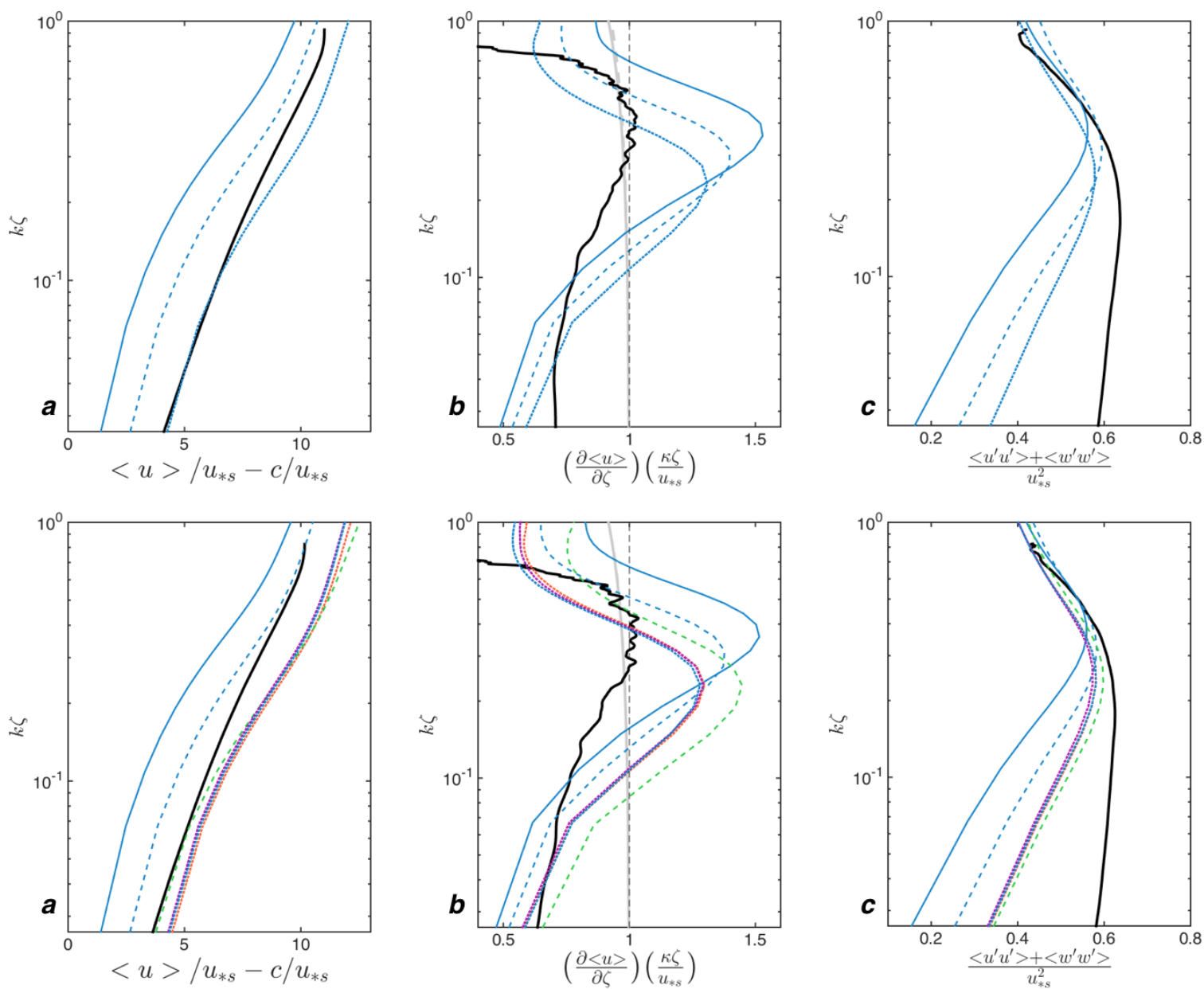

Figure 1.3: Mean normalized wind speed (a), wind shear (b), and 2D turbulent kinetic energy (c) for $c / u_{* s}=1.6$ (top panel) and $c / u_{* s}=1.4$ (bottom panel). Black lines show observational (PIV) results, while dark blue lines show LES results for three different surface roughness lengths: $k z_{o 1}$ (solid), $k z_{o 2}$ (dashed), and $k z_{o 3}$ (dotted). Perturbations include runs 2b (green), 3b (orange), and 3c (purple). All quantities are normalized using the surface friction velocity $u_{* s}$. Grey lines (LES: solid; PIV: dashed) in the middle panels refer to the unperturbed wind shear profile (without waves) accounting for the linear change in total wind stress with height. The results below $k \zeta \approx 0.025$ are omitted because the PIV profiles are strongly affected by viscosity and small scale waves, and above $k \zeta \approx 0.824$ due to noisiness. 

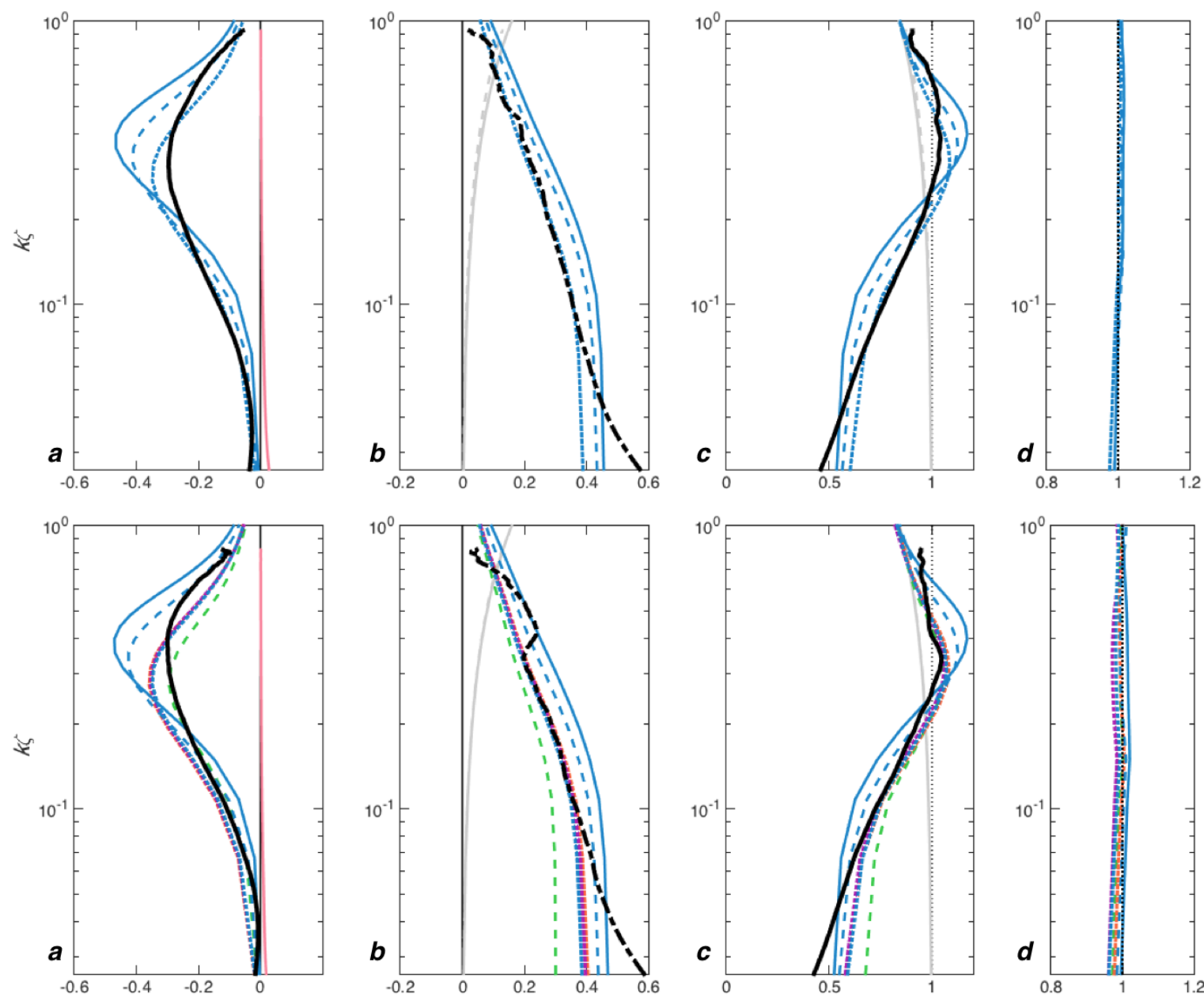

Figure 1.4: Horizontally-averaged profile of each normalized wind stress component for $c / u_{* s}=1.6$ (top panel) and $c / u_{* s}=1.4$ (bottom panel), with components shown as (a) wave-coherent stress $\left(<\tau^{w}>/ \tau_{s}\right)$ and viscous stress (pink, PIV only), (b) pressure stress $\left(<\tau^{p}>/ \tau_{s}\right)$ and vertically integrated pressure gradient $\left(\frac{\partial P}{\partial x} \zeta / \tau_{s}\right.$; light grey), (c) turbulent stress $\left(\left(\left\langle\tau^{t}\right\rangle / \tau_{s}\right)\right.$ and linearly decreasing total wind stress $\left(\tau_{\text {tot }} / \tau_{s}=1-\frac{k \zeta}{2 \pi}\right.$; light grey),

and (d) sum of all stresses including $\frac{\partial P}{\partial x} \zeta / \tau_{s}$ for each LES run, close to unity at all heights above the surface as expected. Black lines show observational (PIV) results, while dark blue lines show LES results for three different surface roughness lengths, with $k z_{o 1}$ (solid), $k z_{o 2}$ (dashed), and $k z_{o 3}$ (dotted). Perturbation include runs $2 \mathrm{~b}$ (green), 3b (orange), and 3c (purple). The PIV pressure stress in (b) (dot-dashed) is estimated as the residual of all other stresses assuming linearly decreasing total wind stress. The results below $k \zeta \approx 0.025$ are omitted because the PIV profiles are strongly affected by viscosity and small scale waves, and above $k \zeta \approx 0.824$ due to noisiness. 

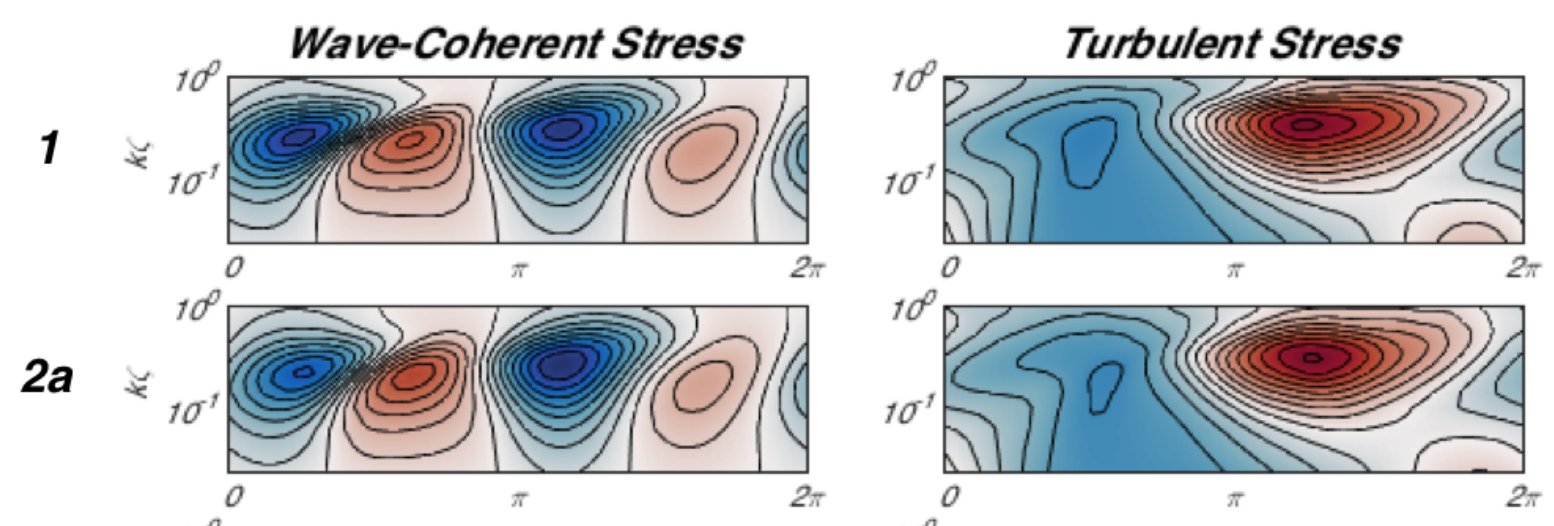

$2 b$
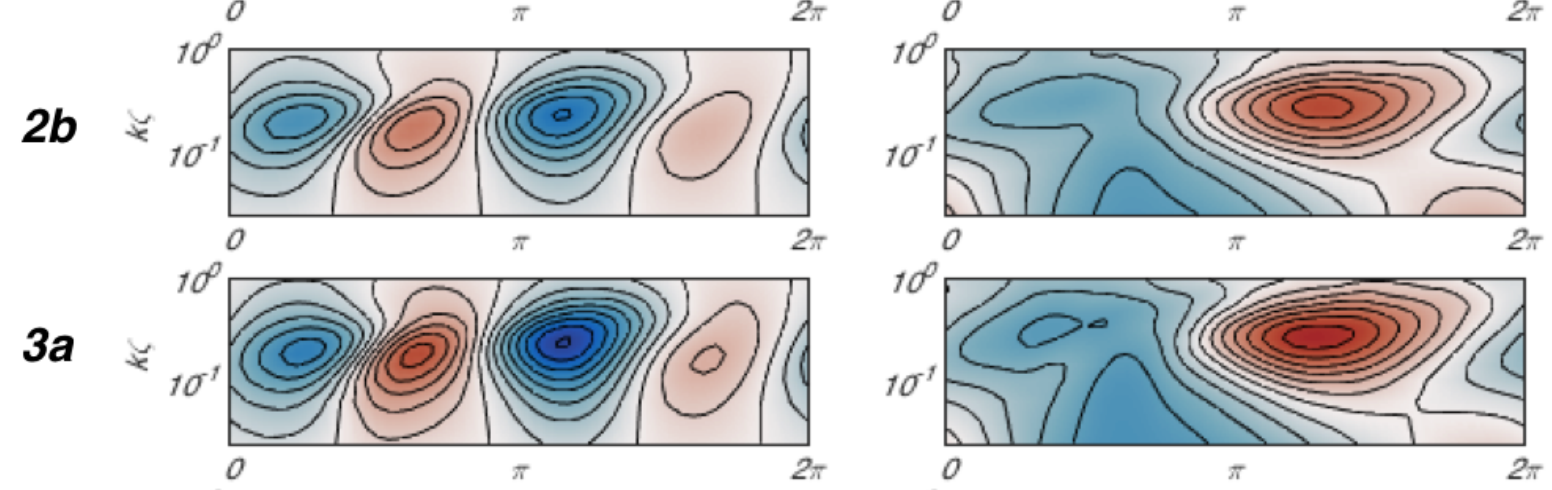

$3 b$
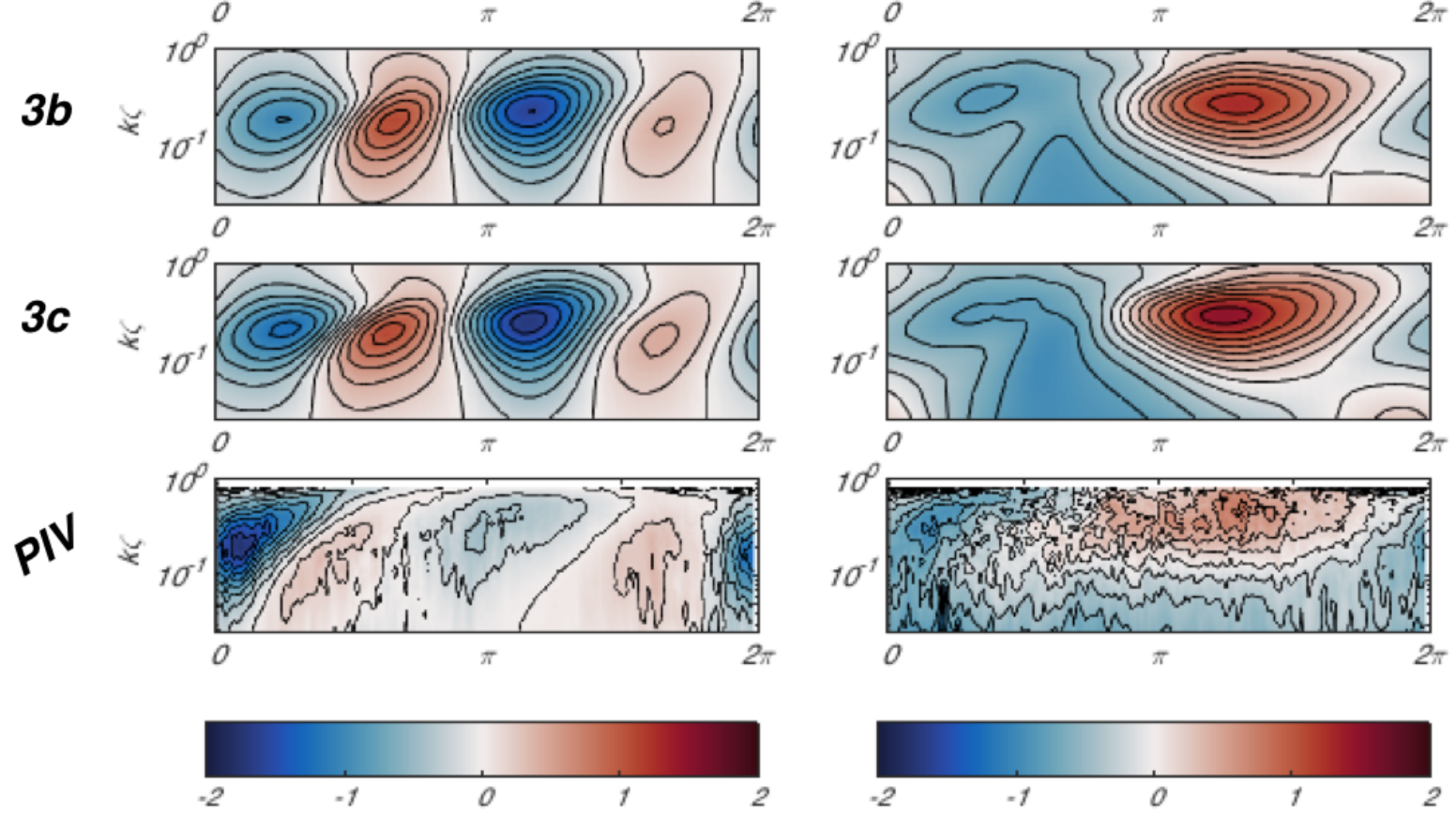

Figure 1.5: Normalized phase-averaged mapped fields of wave-coherent stress and excess turbulent stress (turbulent stress minus total wind stress) for LES and PIV. Each panel is labeled corresponding to its run number (see Table 1). Observations are shown in the bottom panel. All results are normalized by the surface stress, $\tau_{s}=u_{* s}^{2}$. 

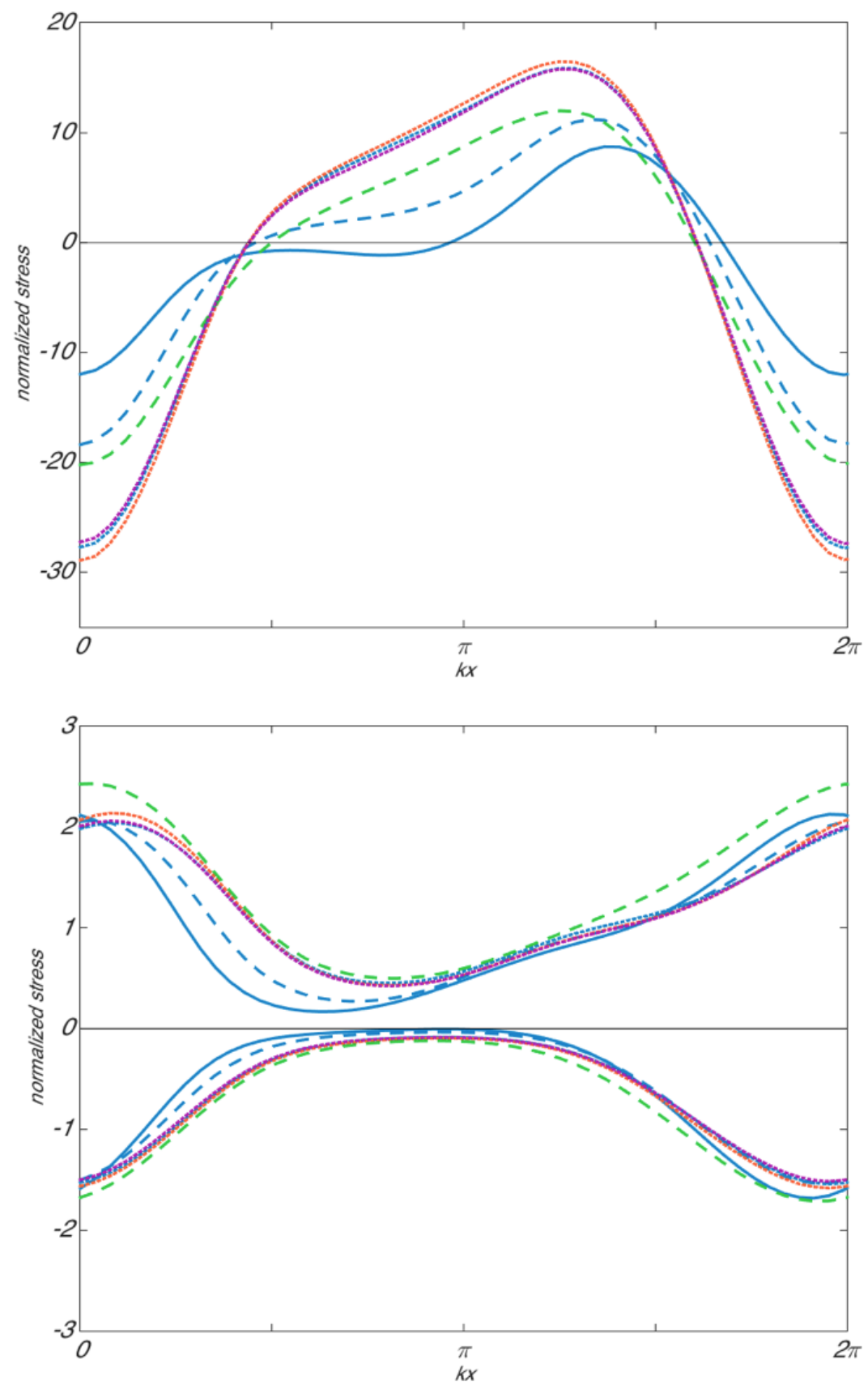

Figure 1.6: Surface stress components for $c / u_{* s}=1.4$, with (1) pressure contribution, and (2) turbulent normal stress (above zero), and turbulent tangential stress (below zero). Dark blue lines show LES results for three different surface roughness lengths, with $k z_{o 1}$ (solid), $k z_{o 2}$ (dashed), and $k z_{o 3}$ (dotted). Perturbations include runs $2 \mathrm{~b}$ (green), 3b (orange), and 3c (purple). All results are normalized by the total wind stress, or surface friction velocity squared, $\tau^{\text {wind }}=u_{* s}^{2}$ 


\title{
Wind turbulence over misaligned surface waves and air-sea momentum flux. Part I: Waves following and opposing wind
}

\author{
Nyla Husain ${ }^{1 *}$, Tetsu Hara ${ }^{1}$, and Peter P. Sullivan ${ }^{2}$
}

Prepared for submission to Journal of Physical Oceanography.

\footnotetext{
${ }^{1}$ Graduate School of Oceanography, University of Rhode Island, Narragansett, Rhode Island ${ }^{*}$ Corresponding author email: nylahusain@uri.edu

${ }^{2}$ National Center for Atmospheric Research, Boulder, Colorado
} 


\subsection{Abstract}

Air-sea momentum and scalar fluxes are strongly influenced by the coupling dynamics between turbulent winds and a spectrum of waves. Because direct field observations are difficult, particularly in high winds, many modeling and laboratory studies have aimed to elucidate the impacts of the sea state and other surface wave features on momentum and energy fluxes between wind and waves as well as on the mean wind profile and drag coefficient. Opposing wind is common under transient winds, for example under tropical cyclones, but few studies have examined its impacts on air-sea fluxes. In this study, we employ a large eddy simulation for wind blowing over steep sinusoidal waves of varying phase speeds, both following and opposing wind, to investigate impacts on the mean wind profile, drag coefficient, and wave growth/decay rates. The airflow dynamics and impacts rapidly change as the wave age increases for waves following wind. However, there is a rather smooth transition from the slowest waves following wind to the fastest waves opposing wind, with gradual enhancement of a flow perturbation identified by a strong vorticity layer detached from the crest despite the absence of apparent airflow separation. The vorticity layer appears to increase the effective surface roughness and wave attenuation rate substantially for faster waves opposing wind. Our results also suggest that the reversal of energy flux direction - from waves to wind - for waves opposing wind contributes significantly to the enhanced drag coefficient.

\subsection{Introduction}

There is a continued interest in improving the prediction and parameterization of momentum, energy, heat, and gas exchanges between the ocean and atmosphere. 
It is well known that the coupling between wind and ocean surface waves modifies turbulent air-sea fluxes, thereby playing a substantial role in the development of weather and climate patterns forecast by numerical models (Cronin et al. 21). Yet, many uncertainties remain as to how wind-wave coupling impacts air-sea momentum and scalar fluxes, as well as wave growth and dissipation, under a range of sea states (Sullivan and McWilliams 91).

Coupled ocean, atmosphere, and wave models often struggle to accurately forecast the evolution of wave fields, ocean currents, storm surge, and weather events without accounting for the effects of wind-waves and swell on the wind stress or drag coefficient, $C_{d}$ (Cronin et al. 21, Donelan et al. 28, Fan et al. 39, Moon et al. $73,74,75$, Reichl et al. 83). Most existing models rely on an empirically-derived bulk $C_{d}$ that uses Monin-Obukhov similarity relationships to define the wind stress as a simple function of wind speed in neutral conditions (e.g. Edson and Fairall 35, Large and Pond 64). To account for the wave (sea state) effects in a relatively simple manner, many studies have addressed the impacts of wave parameters such as wave age $\left(c / u_{* s}\right.$, where $c$ is the wave phase speed and $u_{* s}$ is the wind friction velocity) and wave steepness ( $a k$, where $a$ is the wave amplitude and $k$ is the wavenumber) on the wind stress and sea surface roughness (Banner 4, Banner and Melville 5, Belcher et al. 8, Donelan et al. 30, Edson et al. 36, Fairall et al. 38, Makin and Kudryavtsev 66).

A number of more complex models and parameterizations have been developed to account for the effects of wave-driven turbulent processes near the wavy surface under strong wind forcing. Such processes include airflow separation over breaking waves (Donelan et al. 27, Kudryavtsev and Makin 57, Kukulka and Hara 62, Kukulka et al. 63, Makin and Kudryavtsev 67, Mueller and Veron 76, Suzuki et al. 94), 
ejection of sea spray and spume from wave crests (Andreas 2, Kudryavtsev and Makin 60, Richter and Sullivan 85, Veron 102), enhanced bubble production, air entrainment and gas transfer by breaking waves (Deike et al. 23, Deike and Melville 24), and near-surface ocean currents driven by waves (Teixeira 97, Wang et al. 105), to name a few. A variety of parameterizations have also been developed to represent the effects of wind-wave misalignment on wave growth and dissipation (e.g. Ardhuin et al. 3, Kudryavtsev and Makin 59, Meirink et al. 69, Tolman and Chalikov 98) and on the surface wind stress and drag coefficient (Bourassa et al. 10, Grachev et al. 46, Roekel et al. 86, Suzuki et al. 95).

Several studies have developed sea-state dependent parameterizations of wind stress (or $C_{d}$ ) in complex sea states and extreme winds (Donelan et al. 28, Fan et al. 39, Hara and Belcher 49, 50, Moon et al. 74, Reichl et al. 83). Because field observations in these conditions are limited, the physical mechanisms for the observed leveling off (or reduction) of $C_{d}$ in extreme winds remain to be fully explained (e.g., Black et al. 9, Holthuijsen et al. 55, Powell et al. 82). Donelan [26] and Donelan et al. [27] suggest that under strong wind forcing, airflow separation over steep waves may be an important feature that modifies $C_{d}$ by causing airflow to skip over troughs and reattach at each crest, rendering the troughs invisible to the wind and reducing the overall sea surface roughness. Following from these studies, Peirson and Garcia [79] and Grare et al. [47] use field and laboratory results to emphasize the importance of the wave steepness $(a k)$ and the pressure wave slope correlation in determining the form drag and wave growth rate. They observe a reduced wave growth rate with increasing $a k$, and a phase shift of the pressure field which suggests reattachment of separated airflow onto the windward face of the following wave. 
Earlier studies have described airflow separation as a process which occurs only after waves have started breaking (Banner 4, Banner and Melville 5, Belcher and Hunt 7, Reul et al. 84), but recent laboratory studies using particle image velocimetry (PIV) in a wind-wave flume have captured intermittent airflow separation events over both breaking and non-breaking waves (Buckley and Veron 12, 14, Savelyev et al. 87, Troitskaya et al. 99, Veron et al. 103). The PIV results from a recent study by Savelyev et al. [87] in a wind-wave tank capable of sustaining strongly energetic wave fields (i.e. high $a k$ ) show dampened turbulent kinetic energy in the water directly below strongly forced waves possibly due to enhanced airflow separation and reduced $C_{d}$. In laboratory observations, intermittent airflow separation events have two main features in common: detachment of a high vorticity shear layer from the steep wave crest, and weak stagnant velocity in a region below (the "dead zone"). In this region, recirculation (closed streamlines) may be present in the phase-averaged flow fields shown in a reference frame moving with the wave phase speed. The recirculation patterns associated with airflow separation are distinct from those present over more mature waves for which a coherent critical layer height (where wind speed is equal to the wave phase speed) is visible above the wavy surface. It should be noted that the zero-wall stress criterion traditionally used to define separation points is not generally applicable when the fluid velocity is not zero at the boundary, e.g., flows over rotating cylinders. Boundary movement following or opposing the overlying airflow modulates separation (e.g., Gad-el Hak and Bushnell 40).

Numerical turbulence models have been used to gain more insight into the wind turbulence over surface waves. These have included direct numerical simulations (DNS; Druzhinin et al. 33, Sullivan et al. 92, Yang and Shen 107, 108, Yang 
et al. 109) and large eddy simulations (LES; Hao and Shen 48, Hara and Sullivan 51, Husain et al. 56, Sullivan et al. 89, 90, Suzuki et al. 94), both of which have enabled a detailed exploration of the flow characteristics that may modify the wind stress, wave growth and dissipation over a wide range of wave parameters (e.g., wave age, wave steepness). Some have been able to reproduce laboratory observations reasonably well (Husain et al. 56, Sullivan et al. 90, Troitskaya et al. 100). In particular, Husain et al. [56] have compared LES with the PIV results of Buckley and Veron [12] for airflow over a train of steep, strongly forced waves in a laboratory wind-wave flume. Both model and laboratory results exhibit a phase-averaged signature of frequent airflow separation. The reasonable validation of LES results against observations by Husain et al. [56] provides the basis for the current study to use LES to explore the airflow turbulence (occurrence and effects of intermittent airflow separation, in particular) over an extended range of wind and wave conditions that are expected in open ocean conditions.

In Part I of this study, we use the identical LES approach to explore a range of wave ages for waves following and opposing wind. A number of previous laboratory studies have addressed wind opposing waves. Young and Sobey [110] have measured pressure fields to be nearly symmetric about the wave crest, similar to potential flow theory and consistent with previous field observations (Hasselmann and Bösenberg 53, Snyder et al. 88). Peirson et al. [80] and Mitsuyasu and Yoshida [70] have measured the evolution of waves opposing wind and have found considerable wave decay, consistent with the results of previous numerical simulations using Reynoldsaveraged Navier-Stokes equations (Al-Zanaidi and Hui 1, Cohen 20, Harris et al. 52, Mastenbroek 68). Donelan [25] has also measured wave decay using a pressureslope correlation, finding that the strong pressure signal in opposing wind can 
result in substantial form drag despite the absence of a noticeable phase shift in the dominant pressure field. A more recent modeling study of wind opposing waves by Cao et al. [16] using the wall-resolved LES finds a nearly symmetric pressure signal varying with $a k$ and $c / u_{*}$, and wave decay rates comparable to parameterizations derived from a number of the aforementioned laboratory results (see their Figures 18 and 19). They suggest that the flow dynamics in opposing wind are mainly governed by linear processes that drive the dominant in-phase component of the flow, while nonlinear processes very close to the surface produce a small out-of-phase component that results in the modification of the form drag and wave decay rate.

Despite the existing literature, the physical mechanisms that modify the wind stress, form drag, and wave decay for waves in opposing winds are still not clearly understood. In particular, few studies have explored how waves opposing wind may enhance the effective surface roughness length and the drag coefficient, even though waves opposing wind are quite common and significantly modify the drag coefficient in tropical cyclone conditions (Chen and Curcic 18, Chen et al. 19, Reichl et al. 83). The goal of this study is to expand upon the existing literature to address how opposing wind and waves impact the turbulence in the airflow that modifies these parameters, particularly for steep waves over a range of wave phase speeds, both positive and negative, relative to wind forcing $\left(c / u_{* s}\right)$.

\subsection{Methods}

\section{Large eddy simulation setup}

We use the LES methodology identical to previous studies (Hara and Sullivan 51, Husain et al. 56, Sullivan et al. 90, 93), which employs a pressure-driven channel 
flow over a wavy surface propagating through a rectangular domain with doubly periodic horizontal boundaries. We define time $t$, along-wave $x$-coordinate, crosswave $y$-coordinate, and vertical coordinate $z$ pointing upward with $z=0$ at the mean water surface. Velocities $(u, v, w)$ are in the $(x, y, z)$ directions.

Our LES uses a wave-following vertical coordinate transformed from the physical (Cartesian) coordinate such that the computational grid follows the shape of the waves close to the surface:

$$
z=\zeta^{L E S}+h(x, t)\left(1-\frac{\zeta^{L E S}}{l_{\zeta}}\right)^{3}
$$

where $z$ is the physical vertical coordinate, $\zeta^{L E S}$ is the computational (LES) vertical coordinate, $h(x, t)$ is the time-varying wave shape, and $l_{\zeta}$ is the vertical height of the domain. This transformation makes the LES vertical coordinate equal to the physical vertical coordinate at the flat top boundary, where the free-slip condition is imposed (no tangential stress, $w=0$ ).

For most conditions in our study, we consider a linear monochromatic surface wave train with $h(x, t)=a \cos (k x-\omega t)$, where $a$ is the wave amplitude, $k$ is the wavenumber, $\omega$ is the angular frequency, and $c=\omega / k=\sqrt{g / k}$ is the wave phase speed. The wave orbital velocities, $u=a \omega \cos (k x-\omega t)$ and $w=a \omega \sin (k x-\omega t)$, are used as the surface boundary condition for the LES. We choose a linear wave shape because we have found that the observed phase averaged wave shape of wind waves (with their wave phase determined using the Hilbert transform) is very close to the linear wave shape (Husain et al. 56). However, for one condition, we apply the first-order nonlinear Stokes wave solution so that the bottom wave shape includes the second harmonic, $h(x, t)=a \cos (k x-\omega t)+\frac{1}{2} k a^{2} \cos (2 k x-2 \omega t)$, while 
the phase speed and wave orbital velocities remain identical to those of the linear deep water wave theory.

The dimensions of the computational domain are $l_{x} \times l_{y} \times l_{\zeta}$ where $l_{x}=$ $l_{y}=5 \lambda$ and $l_{\zeta}=2.435 \lambda$, with $\lambda=2 \pi / k$ as the wavelength. It is discretized with $\left(N_{x}, N_{y}, N_{\zeta}\right)=(256,256,256)$ grid points, making the horizontal resolution $\Delta x=\Delta y=0.01953 \lambda$. The vertical spacing ratio gradually increases away from the surface so that the ratio between neighboring cells is held constant at 1.0028, with the first point off the water surface located at $\zeta_{1}^{L E S}=0.0065 \lambda$. In contrast to previous studies (Hara and Sullivan 51, Husain et al. 56, Sullivan et al. 90), we have expanded the vertical domain height from $l_{\zeta}=\lambda$ to $l_{\zeta}=2.435 \lambda$, with double the vertical grid points (from $N_{\zeta}=128$ to $N_{\zeta}=256$ ) to allow for more prominent vertical motions away from the surface associated with higher $c / u_{* s}$ and opposing wind conditions (see Subsection d).

\section{Wind forcing}

The LES setup models an environment similar to that of a wind-wave flume, where an externally imposed horizontal pressure gradient $\partial P / \partial x$ is balanced by a surface wind stress such that $\tau_{s}=(\partial P / \partial x) l_{\zeta}$. Here, the stress and the pressure have been divided by air density $\rho_{a}$ and have dimensions of velocity squared. The surface friction velocity is defined as $\left|\tau_{s}\right|=u_{* s}^{2}$. For waves following (opposing) wind at a $0^{\circ}\left(180^{\circ}\right)$ alignment angle, wind forcing is applied in the $x$-direction with a negative (positive) external pressure gradient $\partial P / \partial x$ that yields a negative (positive) surface stress $\tau_{s}$. Because the pressure gradient is constant with height, the total wind stress magnitude decreases linearly to zero at the ceiling where the free-slip condition is imposed. Recent studies have compared LES model 
results to laboratory measurements of turbulent airflow in a wind-wave flume under strongly forced conditions (e.g., Husain et al. 56, Sullivan et al. 90), finding that LES can accurately reproduce the mean wind profile, momentum flux budget, and phase-averaged turbulent flow fields using an idealized wind-wave channel setup. Furthermore, these results show that the turbulence fields in the wave boundary layer (where they are modified by surface waves) are not significantly affected by the linearly decreasing wind stress in contrast to those in the constant stress layer in open ocean conditions.

\section{Subgrid scale and surface roughness parameterizations}

Turbulent flow in LES is spatially filtered such that dominant-scale turbulence is resolved while subgrid-scale (SGS) fluxes below a filter threshold are parameterized using a conventional TKE-closure SGS parameterization described in more detail in Moeng [71], Sullivan et al. [93], and Moeng and Sullivan [72], and used by Hara and Sullivan [51], Sullivan et al. [90], Husain et al. [56], and a number of other studies.

Along the wavy surface, the local instantaneous tangential stress is parameterized based on the local instantaneous mean wind shear (determined from the difference between the surface tangential water velocity and the tangential wind velocity at the first grid point off the surface) by applying the law of the wall (a log profile) with a prescribed background surface roughness length $z_{o b}$. The parameter $z_{o b}$ represents the bulk effect of viscosity (which is more important in laboratory conditions) and subgrid roughness elements such as higher frequency waves (which dominate in open ocean conditions) on the local frictional stress.

In previous studies (Hara and Sullivan 51, Sullivan et al. 90) the normalized 
background surface roughness has been set at $k z_{o b}=2.7 \times 10^{-3}$ to represent typical strongly forced wind wave conditions in a laboratory setting. In open ocean conditions with a spectrum of waves, $k z_{o b}$ likely varies significantly depending on wind speed, the scale of resolved waves of interest, and other environmental factors such as surfactants. It is also expected that $z_{o b}$, which represents unresolved wave effects, should vary with the phase of the resolved waves because shorter waves are known to be modulated by longer waves (e.g., Gent 43, Gent and Taylor 44, Kudryavtsev and Makin 58).

In our recent study (Husain et al. 56) we have systematically investigated how varying $k z_{o b}$ affects the airflow turbulence over steep waves $(a k=0.27)$ in strongly forced conditions. The results show that the flow field is quite sensitive to the $z_{o b}$ value specified near the crest; a higher $z_{o b}$ increases the frequency of intermittent airflow separation events and enhances the resulting signature in the phase averaged flow fields. However, the airflow is hardly affected if the $z_{o b}$ value is altered away from the crest. In the same study, the effect of wave phase dependent surface drift velocity has been investigated as well. The results indicate that the drift velocity added near the wave crest simply increases the wind speed by the same amount everywhere without affecting the airflow turbulence characteristics, and that the drift velocity added away from the crest has very little impacts.

Since the effects of varying $z_{o b}$ and surface drift have been investigated previously, in this study we keep the normalized background surface roughness held constant at $k z_{o b}=2.7 \times 10^{-3}$ and impose zero surface drift velocity for all simulations. We then focus on investigating the effects of varying wind forcing $c / u_{* s}$ on the wind turbulence, wave form drag, effective surface roughness length (which includes the effect of resolved waves), and drag coefficient. 


\section{Simulations}

While the actual LES of waves opposing wind is performed by reversing the wind direction as described above, in the following sections we differentiate waves following wind and waves opposing wind by the sign of the wave phase speed $c$; that is, the wind always blows in the positive $x$ direction (wind stress $\tau_{s}$ is always negative), and the waves propagate in the positive/negative $x$ direction for following/opposing cases.

In total, we perform simulations for five wave ages in following wind $\left(c / u_{* s}=\right.$ $1.4,2.8,5.6,8.2,11)$ and five cases in opposing wind $\left(c / u_{* s}=-1.4,-2.8,-5.6,-8.2,-11\right)$ with a wave slope held constant at $a k=0.27$. For wind following waves at $c / u_{* s}=1.4$, we include results for a Stokes wave as described in Subsection a. This range of wave ages and the wave slope are similar to that of Sullivan et al. [89] for waves following wind. We choose to focus on a wave slope at the steeper end of those observed in the field because we are particularly interested in the impacts of intermittent airflow separations (or separation-like flows, as described later) that are more common with steep waves (Donelan et al. 27, see their Figure 6), and because this study is motivated by its potential application in tropical cyclone conditions, where waves opposing wind are common and often steep.

Each simulation is run for approximately 130,000 time steps and averaged over the last 60,000 time steps after the wind field has reached a statistically steady state. Sullivan et al. [93] and Sullivan et al. [90] provide a full description of the LES algorithm and numerical methods used to solve the governing equations. See Table 2.1 for more details on the conditions simulated in this study. 


\section{Data analysis}

For our data analysis, we first map the LES coordinate $\zeta^{L E S}$ back to the $z$ coordinate, and then introduce a horizontal coordinates $\xi$ that moves with the wave phase speed

$$
\xi=x-c t
$$

Some initial analyses are made in the rectangular $(\xi, z)$ coordinate in which the waves are no longer time-varying. However, most results are analyzed after the $z$ axis is re-mapped to a vertical wave following coordinate $\zeta$ that exponentially approaches toward the $z$ coordinate (Hara and Sullivan 51):

$$
z=\zeta+a \cos (k \xi) e^{-k \zeta}
$$

for a linear wave or

$$
z=\zeta+a \cos (k \xi) e^{-k \zeta}+k a^{2} \cos (2 k \xi) e^{-2 k \zeta}
$$

for the Stokes wave, with the Jacobian of coordinate transformation,

$$
J=\frac{\partial \zeta}{\partial z}
$$

In this coordinate system, we employ a triple decomposition to separate all quantities into mean, turbulent, and wave-coherent components, similar to previous studies (e.g., Buckley and Veron 12, Chalikov and Rainchik 17, Hara and Sullivan 51, 
Sullivan et al. 92) using

$$
\psi=\bar{\psi}+\psi^{\prime}=\langle\psi\rangle+\widetilde{\psi}+\psi^{\prime}
$$

where $\bar{\psi}$ is the wave phase average (average in $t$ and $y$ ), $\langle\psi\rangle$ is the horizontal mean (average in $\xi$ ), $\widetilde{\psi}$ is the wave-coherent fluctuation component, and $\psi^{\prime}$ is the turbulent fluctuation component. This approach allows us to define wave-coherent and turbulent fluxes as separate components of the total wind stress. Specifically, the horizontally averaged $x$-momentum equation may be expressed as

$$
\frac{\left\langle\tau^{w}\right\rangle+\left\langle\tau^{p}\right\rangle}{\tau_{s}}+\frac{\left\langle\tau^{t}\right\rangle}{\tau_{s}}+\frac{\frac{\partial P}{\partial x} \zeta}{\tau_{s}}=1
$$

where $\left\langle\tau^{w}\right\rangle=\langle\tilde{u} \tilde{W}\rangle$ is the wave-coherent stress and $\left\langle\tau^{t}\right\rangle=\left\langle u^{\prime} W^{\prime}\right\rangle$ is the turbulent stress that includes both resolved and parameterized subgrid-scale stresses. Notice that these stresses are defined using a contravariant vertical velocity perpendicular to a constant $\zeta$ surface

$$
W=\frac{1}{J} u \frac{\partial \zeta}{\partial x}+w
$$

such that $u W$ represents flux of $x$-momentum across the constant $\zeta$ surface due to the advective velocity $W$ (Hara and Sullivan 51). The pressure (form) stress

$$
\left\langle\tau^{p}\right\rangle=\left\langle\frac{1}{J} \bar{p} \frac{\partial \zeta}{\partial x}\right\rangle
$$

represents momentum flux due to pressure applied on a tilted constant $\zeta$ surface. The sum of the three stress components is equal to the total wind stress $\left\langle\tau^{t o t}\right\rangle=$ $\left\langle\tau^{w}\right\rangle+\left\langle\tau^{p}\right\rangle+\left\langle\tau^{t}\right\rangle$, whose magnitude linearly decreases from the surface. At the wavy surface $(\zeta=0)$ the wave coherent stress $\left\langle\tau^{w}\right\rangle$ is zero and the total wind stress 
$\tau_{s}$ is a sum of the pressure form $\operatorname{drag}\left\langle\tau^{p}\right\rangle$ and the (subgrid) turbulent stress $\left\langle\tau^{t}\right\rangle$; the latter represents the momentum flux into unresolved waves and the surface viscous stress contribution. For more details on the triple decomposition and the derivation of the continuity, momentum, and energy equations in this mapped coordinate, refer to Hara and Sullivan [51].

\subsection{Results and discussion}

\section{Two-dimensional phase averaged airflow above waves following wind}

In this section all flow fields presented are normalized by $u_{* s}$ and $k$. Figures 2.1 and 2.2 display the two-dimensional phase-averaged flow fields for the cases of $c / u_{* s}=1.4,5.6$, and 11 (waves following wind, top panels) and $c / u_{* s}=-1.4,-5.6$, and -11 (waves opposing wind, bottom panels). Figure 2.1 includes the streamlines, streamwise velocity $\left((\bar{u}-c) / u_{* s}\right)$, vertical velocity $\left(\bar{w} / u_{* s}\right)$, and pressure $\left(\bar{p} / u_{* s}^{2}\right)$. In the rightmost column, the surface profiles are shown for the total normal stress (pressure plus the turbulent normal stress), pressure only, and the turbulent tangential stress. Figure 2.2 includes the TKE $\left(\bar{e} / u_{* s}^{2}=\left(\overline{u^{\prime} u^{\prime}}+\overline{v^{\prime} v^{\prime}}+\overline{w^{\prime} w^{\prime}}\right) /\left(2 u_{* s}^{2}\right)\right)$, dissipation rate $\left(\epsilon /\left(k u_{* s}^{3}\right)\right)$, and the spanwise vorticity $\left(\bar{\omega}_{y} /\left(k u_{* s}\right)=\left(\frac{\partial \bar{u}}{\partial z}-\frac{\partial \bar{w}}{\partial x}\right) /\left(k u_{* s}\right)\right)$. All the quantities are plotted in the rectangular $(\xi-z)$ coordinate that moves with the wave, so that the phase averaged flow field is independent of $t$. In addition, the TKE, $\epsilon$, and $\bar{\omega}_{y}$ are plotted in the mapped $(\xi-\zeta)$ coordinate (Figure 2.2, right three panels) with the vertical axis in a log scale so that the flow fields very close to the wavy surface can be clearly observed. Wind is blowing from left to right, and waves are propagating from left to right (right to left) in the following (opposing) cases (before the coordinate transformation). Note that the streamline field has 
been constructed from $\bar{u}-c$ and $\bar{w}$.

First, we examine the cases of waves following wind (top three panels of Figures 2.1 and 2.2). In the case of strongly forced waves at $c / u_{* s}=1.4$ (topmost panels), contours of constant $\bar{u}-c$ expand away from the surface downstream of the crest with reduced velocity and a small recirculation bubble on the leeward side of the crest (Figure 2.1a,g). Inside this bubble, the TKE, dissipation rate, and vorticity are all significantly reduced (Figure 2.2a,g,m, and more clearly seen in Figure 2.2A, G,M in the mapped coordinate with the vertical log scale). The high vorticity layer at the wave crest is detached from the surface and extends downstream of the crest above the recirculation bubble (Figure $2.2 \mathrm{~m}, \mathrm{M}$ ), indicating large streamwise velocity just above this high vorticity layer. The elevated TKE above the crest appears to be advected by the detached high velocity and intensifies, extending just above the high vorticity layer (Figure 2.2a,A). These patterns are identical to the LES results presented in Husain et al. [56] for the same wave age $\left(c / u_{* s}=1.4\right)$ and wave slope $(a k=0.27)$.

Buckley and Veron [12] have been able to demonstrate with high-resolution particle image velocimetry (PIV) that near-surface wind flow patterns are modified by transient, sporadic detachment of airflow from the crests of steep, young waves. Instantaneous measurements of $u$ and $w$ over laboratory waves capture a layer of enhanced spanwise vorticity developing and ejecting away from the wave crest, resulting in weak, stagnant, sometimes negative airflow in the trough (see Buckley and Veron 12, their Figure 6). Previous LES results (e.g. Hara and Sullivan [51], Sullivan et al. [90], and a more recent comparison between these observations and LES by Husain et al. [56] have also found that intermittent airflow separation events can occur frequently over steep, non-breaking waves, and their phase-averaged 
character in observations can be well reproduced in LES. This suggests that while these separation events are not persistent in either the observations or LES, they occur frequently enough in steep, strongly forced conditions to leave a signature in the phase-averaged flow fields.

These intermittent airflow separation events also affect the phase-averaged pressure field (Figure 2.1s,A). It is well known that the pressure-wave slope correlation determines the form drag and the wave growth rate (e.g. Grare et al. 47, Peirson and Garcia 79), but the role of airflow dynamics in shifting the phase and magnitude of maximum surface pressure is still not well understood. The LES results of Sullivan et al. [90] associate the location and magnitude of maximum pressure with reattachment of detached flow onto the windward face of the following wave. Our results also suggest that the reattachment of the separated flow influences the location of the high pressure on the windward face of the wave (Figure 2.1s,A) and the resulting form drag and wave growth rate (discussed in Subsection g).

As the wave age $\left(c / u_{* s}\right)$ increases for waves following wind, the recirculation bubble (or "cat's eye" pattern) grows larger (see expanding blue streamlines in Figure 2.1b,c), indicating that the critical layer expands away from the surface with increasing wave age (note that the wind speed is equal to the wave phase speed at the top of the critical layer). At the same time, the signature of intermittent airflow separation (reduced TKE, $\epsilon$, and $\bar{\omega}_{y}$ inside the recirculation bubble; layers of enhanced TKE, $\epsilon$, and $\bar{\omega}_{y}$ separated from the wave crest and extended above the bubble) rapidly diminish at higher wave ages (Figure 2.2B-C,H-I,N-O). The enhanced $\epsilon$ and $\bar{\omega}_{y}$ remain attached to the surface and appear to shift upstream from the crest to the trough region (Figure 2.2H-I,N-O)). Consistent with the trends shown in Sullivan et al. [89], the near-surface vertical velocity $\bar{w}$ becomes 
more negatively correlated with the local wave slope as the wave age increases (Figures 2.1n,o). Sullivan et al. [89] speculate that these shifting patterns could be due in part to critical layer dynamics that generate waves via shear flow at the height where wind speed is equal to the wave phase speed. They also suggest that faster waves produce an effect more closely associated with a wave driving (or "boundary pumping") regime, whereby the undulation of the wavy surface drives near-surface $\bar{w}$ via the wave's surface tilt interacting with the wave phase speed.

Unlike the slow wave case $\left(c / u_{* s}=1.4\right)$, the region of low pressure for intermediate and faster waves $\left(c / u_{* s}=5.6,11\right)$ occurs along the leeward face of the wave rather than at the top of the crest (Figure 2.1t,u,A), potentially associated with a non-separated "sheltering effect" (e.g. Belcher and Hunt 6, 7). The magnitude of the pressure variation diminishes (Figure 2.1A), and so too do the wave growth rate and form drag as the wave age increases (discussed in Subsection g).

\section{Two-dimensional phase averaged airflow above waves opposing wind}

Next, we examine the cases of waves opposing wind (bottom panels in Figure 2.1 and 2.2). One immediately notices that the flow field is dominated by a strong pressure perturbation (Figure $2.1 \mathrm{v}, \mathrm{w}, \mathrm{x}$ ) in phase with $-h$ (negative of the wave elevation), and a strong vertical velocity perturbation (Figure 2.1p,q,r) in phase with $\partial h / \partial x$ (the wave slope). This result is consistent with the wall-resolved LES results of Cao et al. [16] for waves opposing wind, as well as previous laboratory and field measurements (i.e. Snyder et al. 88, Young and Sobey 110, and Hasselmann and Bösenberg 53). Cao et al. [16] show that these flow features are well explained by a simple linear inviscid model. This trend of increasingly stronger along-wave pressure gradient is reminiscent of potential flow, as observed by Young and Sobey 
[110]. However, the pressure field in phase of $-h$ does not contribute to the wave growth rate or the form drag; only the small out-of-phase component does. Cao et al. [16] discuss how the strong turbulence very near the surface plays an important role in determining the magnitude of this component.

Similarly, our results show that the near surface turbulence fields contain strong wave induced perturbations. The most notable feature near the wavy surface is that the flow fields of TKE, $\epsilon$, and $\omega_{y}$ are surprisingly similar between the cases of $c / u_{* s}=1.4$ (waves following wind, Figures $2.2 \mathrm{~A}, \mathrm{G}, \mathrm{M}$ ) and $c / u_{* s}=-1.4$ (waves opposing wind, Figure 2.2D,J,P)). In both cases, the common signature of intermittent airflow separation are observed (reduced TKE, $\epsilon$ and $\omega_{y}$ in the "dead zone" downstream of the crest; layers of enhanced TKE, $\epsilon$, and $\omega_{y}$ detached from the crest above). In the case of waves following wind, these features are associated with intermittent airflow separations. However, the airflow does not exhibit apparent separation-like patterns in the case of waves opposing wind because the wind speed at the bottom is positive in a reference frame moving with the wave. Nevertheless, our results at $c / u_{* s}=-1.4$ suggest that a strong wind shear develops above the wave crest and generates large TKE and a detached high vorticity (high wind shear) layer from the crest, which extends over a region of reduced vorticity above the leeward trough. The elevated TKE above the crest is advected by the enhanced streamwise velocity, and shows intensification just above the detached high vorticity layer downstream of the crest.

As the wave speed increases from $c / u_{* s}=-1.4$ to -5.6 and -11 , these flow characteristics remain similar but slowly evolve. The elevation of the detached enhanced layers of TKE, $\epsilon$, and $\omega_{y}$ gradually decreases, and the location of the region of reduced TKE, $\epsilon$, and $\omega_{y}$ slowly moves toward the trough (Figure $2.2 \mathrm{E}-\mathrm{F}, \mathrm{K}-$ 
L,Q-R). This evolution is likely due to the increasing streamwise background flow in the reference frame moving with the wave. Nevertheless, all the flow characteristics associated with the detached high vorticity layer persist.

One surprising feature is that the vorticity $\left(\bar{\omega}_{y}\right)$ is not only reduced, but becomes significantly negative below the detached high vorticity layer as $c / u_{* s}$ becomes more negative (Figure 2.2Q,R). This suggests that the wind shear is negative in this region - that is, wind speed is decreasing with height. In the case of waves following wind, such negative wind shear is usually associated with strongly separating flows above actively breaking waves, but it does not occur in the phase-averaged flow fields over non-breaking waves in our LES simulations. For the case of waves opposing wind, negative wind shear near the trough appears to be more common and persistent, suggesting that the flow perturbations just downstream of the wave crest become stronger as $c / u_{* s}$ becomes more negative, even if they are not associated with apparent airflow separations and their vertical extent is somewhat reduced. Note that the persistence of airflow separations is often associated with a small region of recirculation (closed streamlines distinct from those present over more mature waves, see Figure 2.1b,c) for young waves following wind in a reference frame moving with the wave phase speed (see the streamlines for $c / u_{* s}=1.4$, Figure 2.1a), but this pattern is absent for all waves opposing wind in a reference frame moving with the opposing wave phase speed. Although these streamline patterns are absent for opposing waves, the flow perturbation (enhanced negative $\omega_{y}$, reduced TKE, reduced $\epsilon$ ) intensifies regardless.

Although the pressure field appears dominated by the perturbation in phase with $-h$, a significant out-of-phase component exists which is not apparent in the flow fields, but nonetheless results in an increase of the form drag and wave decay 
rate as $c / u_{* s}$ decreases (Figure2.1v-x,C) - see Subsection $g$ for more discussion. It is feasible that the increasingly intensified perturbation downstream of the crest that is apparent in the flow fields of TKE, dissipation rate and vorticity may be (at least partially) responsible for this development.

\section{Instantaneous airflow features}

To demonstrate the transient character of the flow field over a range of $c / u_{* s}$, we display instantaneous snapshots of spanwise vorticity $\omega_{y}$ for $c / u_{* s}=1.4,11$, -1.4 and -11 in Figure 2.3. Slower waves following wind $\left(c / u_{* s}=1.4\right.$, Figure 2.3a) generate enhanced positive vorticity along the windward face of the wave crest. This layer of enhanced vorticity intermittently separates from the leeward face of the crest, coupled with reduced (sometimes negative) vorticity below. The detachment location, trajectory, and magnitude of the enhanced vorticity layer significantly vary from wave to wave.

With faster waves at $c / u_{* s}=11$ (Figure 2.3b) detachment of enhanced positive vorticity from the crest is not apparent. Instead, the vorticity field is dominated by a thin high vorticity layer along the entire wave surface and signatures of ejections and sweeps (motion of air away from and towards the surface), which are characteristic of near-wall turbulent boundary layers over flat walls. The locations of ejections and sweeps appear uncorrelated with the wave phase. These features are consistent with the instantaneous flow fields captured by Buckley and Veron [14] using high-resolution PIV in a wind-wave flume over a similar range of wave ages.

In the case of slow waves opposing wind $\left(c / u_{* s}=-1.4\right.$, Figure $\left.2.3 \mathrm{c}\right)$, the instantaneous flow patterns are quite similar to those of slow waves following wind 
$\left(c / u_{* s}=1.4\right.$, Figure 2.3a), except that the height of the intermittent detached high vorticity layer appears to be suppressed. As the wave speed increases $\left(c / u_{* s}=-11\right.$, Figure 2.3d), the height of the detached high vorticity layer is further reduced but persistent negative vorticity regions appear near the trough, consistent with the phase-averaged vorticity field (Figure 2.2R).

\section{Vertical profiles of horizontally averaged wind fields}

In the following subsections we investigate the vertical profiles (dependence on $\zeta$ ) of wind variables averaged horizontally in the mapped coordinate (mean wind speed, mean wind shear, mean TKE, and the terms in the momentum and energy budget equations) as well as the enhancement of the equivalent surface roughness due to waves and the wave growth (decay) rates for waves following (opposing) wind. In particular, we attempt to explain how these quantities are affected by the physical mechanisms identified in the two dimensional flow analysis in the previous subsections. All the profiles are displayed up to $k \zeta=4$ because the results above this elevation are affected by the reduced wind stress and the LES top boundary.

Figure 2.4a-f display normalized horizontal mean profiles of wind speed $\left(\langle u\rangle / u_{* s}\right)$, wind shear $\left(\frac{\partial\langle u\rangle}{\partial \zeta} \frac{\kappa \zeta}{u_{* s}}\right)$, and TKE $\left(\langle\bar{e}\rangle / u_{* s}^{2}\right)$. The upper panels show results with waves following wind at $c / u_{* s}=1.4,2.8,5.6,8.2$, and 11 (with increasing $c / u_{* s}$ transitioning from warmer to cooler colors), and the lower panels show results with waves opposing wind at $c / u_{* s}=-1.4,-2.8,-5.6,-8.2$, and -11 (with increasingly negative $c / u_{* s}$ transitioning from warmer to cooler colors). The red dot-dashed line in the upper panels represents $c / u_{* s}=1.4$ with a first-order nonlinear Stokes wave including the second harmonic applied to the bottom wave shape.

For waves following wind (Figure 2.4a), the far field wind profiles above the wave 
boundary layer (above about $k \zeta=1$ ) are roughly parallel to, but shifted to the left of, the wind profile over a flat surface with a non-dimensional background surface roughness of $k z_{o b}=2.7 \times 10^{-3}$ (grey lines). Here, the solid grey line represents the wind profile modified by the linearly decreasing wind stress in $k \zeta$, and the dashed grey line is the wind profile for constant stress in $k \zeta$. Since the background roughness length (accounting for the form drag of unresolved small waves and the viscous stress) along the wavy surface is identically set at $k z_{o b}=2.7 \times 10^{-3}$ in all simulations, the decrease of the far field wind speed indicates that the waves enhance the effective roughness length $z_{o}$ (determined by extrapolating the wind profile above the wave boundary layer toward the surface) relative to the background roughness length $z_{o b}$.

Specifically, for each case we roughly estimate $z_{o}$ by horizontally shifting the flat wall wind profile (gray solid line) to match the wind speed profile above the wave boundary layer (matching the wind speed at $k \zeta=4$ for simplicity), then we find the height where the shifted flat wall wind speed becomes zero. We find that the slowest waves $\left(c / u_{* s}=1.4\right)$ produce the largest $z_{o}\left(z_{o} / z_{o b}=4.65\right)$, which rapidly decreases to $z_{o} / z_{o b}=1.58$ as $c / u_{* s}$ increases to 11 (see Table 2.1 for a summary). This trend is consistent with the findings from a DNS study of idealized water waves in Couette flow over a similar range of $c / u_{* s}$ with $a k=0.1,0.2$ (Sullivan et al. 92) and the results of Sullivan et al. [89] for similar $c / u_{* s}$ and $a k$. Note that the case using the second-order Stokes wave for $c / u_{* s}=1.4$ (Run 1.4fs) produces a $25 \%$ higher $z_{o}$ compared to its sinusoidal counterpart (Run 1.4f).

The mean normalized wind shear is shown in Figure 2.4c. Similar to the grey lines for mean wind speed in Figure 2.4a, grey lines here represent the mean wind shear profile unmodified by waves for linearly decreasing wind stress in $k \zeta$ (solid) 
and constant wind stress in $k \zeta$ (dashed). When the wind shear profile deviates to the right (left) of the grey solid line, the wind shear is enhanced (reduced) due to the wave effect. Since the mean wind speed must approach zero at the background roughness height in all simulations $\left(z=z_{o b}\right)$, the shift of the far field wind profile relative to the flat wall profile means that the mean wind shear is modified by waves inside the wave boundary layer. Here, the normalized mean wind shear is defined and plotted such that the area integral of its deviation from the flat wall case is approximately proportional to the deviation of the normalized far field mean wind speed from the flat wall profile (see Hara and Sullivan 51 their Figure 2 and discussion).

For waves following wind, the wave age plays a significant role in the character of the mean wind shear. Slower waves $\left(c / u_{* s}=1.4,2.8\right)$ show enhanced wind shear slightly above the height of the wave crest and reduced wind shear toward the surface. This pattern has been observed for similar wave ages in the LES results of Husain et al. [56], where we describe the enhanced mean wind shear as a signature of frequent airflow separations due to the averaged effect of enhanced vorticity layers ejecting off of wave crests. They also demonstrate that as the wave slope $(a k)$ is reduced, this signature is muted as airflow separations (and enhanced wind shear) become less frequent. Since the contribution of the reduced mean wind shear toward the surface is more significant than that of the enhanced wind shear near the crest height, the far field wind speed decreases and thus the equivalent surface roughness $z_{o}$ increases.

As the wave age increases to $c / u_{* s}=5.6$ and above, the enhancement of the wind shear almost completely disappears, indicating that little to no airflow separations are occurring. The decrease in wind shear toward the surface remains significant, 
but gradually decreases with increasing $c / u_{* s}$. Consequently, the reduction of far field wind speed and the increase of the equivalent surface roughness $z_{o}$ are also less significant.

Note that the normalized mean shear remains slightly above the flat wall case (grey solid line) at the top of the domain $(k \zeta=4)$ for the cases of $c / u_{* s}=5.6$, 8.2 , and 11 , suggesting that the wave effect on the mean shear has not completely disappeared at this height in these cases. If this slightly elevated wind shear persists further above, it is possible that the wind profiles approach closer to the solid grey line at higher elevations and $z_{o}$ may be slightly lower than our estimates, which are made using the wind speed at $k \zeta=4$ (where the effects of the LES top boundary are small).

The mean normalized 3D TKE profiles are shown in Figure 2.4e. For the two slow wave cases the enhancement of TKE is generally located at about the same elevation as the enhancement of the mean wind shear in Figure 2.4c. As $c / u_{* s}$ increases, this enhancement gradually diminishes and completely disappears at $c / u_{* s}=11$. Although small deviations to the TKE profile are present closer to the surface, it becomes more uniform overall and suggests substantially reduced wave impacts.

Next, we examine the case for waves opposing wind. With slow waves at $c / u_{* s}=$ -1.4, the wind speed and wind shear profiles (solid dark red lines, Figures 2.4b,d) in the lower part of the wave boundary layer are quite similar to those for slow waves following wind at $c / u_{* s}=1.4$ (solid dark red lines, Figure $2.4 \mathrm{a}, 4 \mathrm{c}$ ), except that the height of the enhanced wind shear is slightly lower. However, they are significantly different in the upper region. For $c / u_{* s}=1.4$, the wind shear converges to that over a flat surface above around $k \zeta=0.6$, but with $c / u_{* s}=-1.4$ the wind 
shear becomes significantly reduced above around $k \zeta=0.5$ before it converges to the flat wall case near $k \zeta=4$. Consequently the far field wind speed is more reduced and the equivalent roughness of $z_{o} / z_{o b}=7.04$ is significantly larger than that of $c / u_{* s}=1.4$. The mean TKE profile at $c / u_{* s}=-1.4$ is similar to that of $c / u_{* s}=1.4$ with its peak slightly above the height of the peak of the mean wind shear (Figure 2.4f).

As the wave speed increases and $c / u_{* s}$ decreases from -1.4 to -11 , the mean wind shear profile maintains a qualitatively similar character. The wind shear continues to show pronounced enhancement, but its peak shifts closer to the surface and slightly weakens with more negative $c / u_{* s}$. The pattern of reduced wind shear towards the surface slightly increases with faster waves but remains largely unchanged. Above the layer of enhancement, the wind shear reduces much more dramatically and expands much farther away from the surface as $c / u_{* s}$ becomes more negative, suggesting that the vertical extent of the wave impact on mean wind shear increases with increasing wave speed. Cao et al. [16] suggest that the increasingly strong vertical velocity interacts with the mean wind shear to amplify the effect of wave kinematics on the airflow fields as the opposing wave speed increases. Because of this elevated layer of reduced wind shear, the far field wind speed continues to decrease and the effective surface roughness $z_{o}$ significantly increases as $c / u_{* s}$ becomes more negative, reaching to $z_{o} / z_{o b}=31.80$ at $c / u_{* s}=-11$. The mean TKE profile also remains qualitatively similar; the height of the well-defined TKE peak gradually decreases and the peak value slightly decreases as $c / u_{* s}$ becomes more negative (Figure 2.4f).

Similar to the case of faster waves following wind $\left(c / u_{* s}=5.6,8.2\right.$, and 11$)$, the wave effect on the mean wind shear has not completely disappeared at the top 
of the domain $(k \zeta=4)$ for faster waves opposing wind $\left(c / u_{* s}=-5.6,-8.2\right.$, and -11). The wind shear is still below the flat wall case at this height (compared to the grey solid line). If the reduced wind shear persists further above, the wind profiles may deviate further from the solid grey line at higher elevations, which may result in a larger $z_{o}$ than our estimates in Table 2.1.

\section{Momentum budget in mapped coordinate}

The horizontally averaged momentum budget (or wind stress partition) as described in Equation 3.2 is shown in Figures 2.5A,C (upper panels) for $c / u_{* s}=1.4$, 2.8, 5.6, 8.2 and 11 (increasing wave age transitioning from warmer to cooler colors) for waves following wind, and in Figures 2.5B,5D (lower panels) for $c / u_{* s}=-1.4$, $-2.8,-5.6,-8.2$ and -11 (increasingly negative wave age transitioning from warmer to cooler colors) for waves opposing wind. Similar to the mean wind speed and mean wind shear, the solid (dashed) grey lines represent linearly decreasing (constant) total wind stress in $k \zeta$. As discussed earlier, the horizontally averaged total stress $\left\langle\tau^{t o t}\right\rangle$, which linearly decreases from the surface, is equal to the sum of

the pressure stress $\left\langle\tau^{p}\right\rangle$, the wave coherent stress $\left\langle\tau^{w}\right\rangle=\langle\tilde{u} \tilde{W}\rangle$, and the turbulent stress $\left\langle\tau^{t}\right\rangle=\left\langle u^{\prime} W^{\prime}\right\rangle$ (including both resolved and SGS contributions in LES) in the wave boundary layer. Recall that $W$ is a velocity normal to the mapped constant $\zeta$ plane, so these stresses act normal to the wavy surface. The total stress $\left\langle\tau^{t o t}\right\rangle$ profiles shown in Figure 2.5A,B are obtained by adding these three stress components calculated from the LES results, and are (almost) equal to the linearly decreasing stress profile (solid gray line, not visible). This convergence of $\tau^{\text {tot }}$ confirms that the LES results properly satisfy the momentum budget.

The two-dimensional fields of the phase-averaged normalized turbulent stress 
$\overline{\tau^{t}}=\overline{u^{\prime} W^{\prime}}$ and wave-coherent stress $\overline{\tau^{w}}=\tilde{u} \tilde{W}$ are displayed in mapped coordinates for $c / u_{* s}=1.4,5.6$, and 11 in Figure 2.5a-c,g-i (upper panels) and for $c / u_{* s}=-1.4$, -5.6, and -11 in Figure 2.5d-f,j-l (lower panels). Note that the color scale of the turbulent stress is shifted such that red (blue) means the local turbulent stress is larger (smaller) than the normalized surface wind stress (which is -1 ).

For the slow waves following wind $\left(c / u_{* s}=1.4\right.$ and 2.8$)$, the horizontally averaged wave coherent stress is significantly enhanced and positive (upward momentum flux) around the height where the mean wind shear and the TKE are also elevated. The two-dimensional distribution of the wave coherent stress $\tilde{u} \tilde{W}$ (Figure 2.5a) shows that intense upward momentum flux occurs just downstream the crest where the high velocity fluid $(\tilde{u}>0)$ is detached from the surface $(\tilde{W}>0)$, and just downstream the trough where the low velocity fluid $(\tilde{u}<0)$ reattaches toward the surface $(\tilde{W}<0)$. This suggests that the positive wave coherent stress is enhanced by intermittent airflow separation events. To compensate this elevated positive $\left\langle\tau^{w}\right\rangle$, the negative turbulent stress $\left\langle\tau^{t}\right\rangle$ (downward momentum flux) is significantly enhanced at a similar elevation. This enhancement mainly occurs downstream of the trough (Figure 2.5g). The magnitude of the negative pressure stress $\left\langle\tau^{p}\right\rangle$ monotonically increases toward the surface and remains approximately constant at around -0.5 below $k \zeta=0.1$ (Figure 2.5A) - that is, the pressure stress (which is equal to the wave form drag at the surface) accounts for half of the total wind stress. The wave coherent stress $\left\langle\tau^{w}\right\rangle$ becomes negligible below $k \zeta=0.05$ and the turbulent stress is reduced to about half of the wind stress and compensates for the large pressure stress nearer to the surface (Figure 2.5C). For $c / u_{* s}=1.4$, the addition of the Stokes solution to the wave shape enhances the pressure stress (and the form drag at the surface) by $9 \%$ above its sinusoidal counterpart. 
As the wave age increases to $c / u_{* s}=5.6$ and above, the enhanced positive wave coherent stress rapidly diminishes and slightly moves upward (Figure 2.5C). Near the surface the pressure stress still supports roughly half of the wind stress at $c / u_{* s}=5.6$ (Figure $\left.2.5 \mathrm{~A}\right)$. As $c / u_{* s}$ increases more, the magnitude of the pressure stress and the reduction of the turbulent stress gradually diminish but remain significant. At $c / u_{* s}=11$ the pressure stress still accounts for about $1 / 4$ of the total wind stress.

For slow waves opposing wind at $c / u_{* s}=-1.4$, the vertical profiles of the stress components (Figure 2.5B,D, dark red lines) are quite similar to those for the slow waves following wind at $c / u_{* s}=1.4$, Figure $2.5 \mathrm{~A}, \mathrm{C}$, dark red lines). The positive enhancement of $\left\langle\tau^{w}\right\rangle$ and the negative enhancement of $\left\langle\tau^{t}\right\rangle$ are both apparent, but they are slightly weaker and occur at slightly lower elevations. These patterns are accompanied by significant $\left\langle\tau^{p}\right\rangle$ (about $1 / 2$ of the total wind stress) corresponding to the reduction of $\left\langle\tau^{t}\right\rangle$ toward the surface, similar to the following case.

As the wave speed increases and $c / u_{* s}$ decreases to -11 , these patterns remain qualitatively similar, but the positive enhancement of $\left\langle\tau^{w}\right\rangle$ and the negative enhancement of $\left\langle\tau^{t}\right\rangle$ both gradually weaken and move lower in $\zeta$. This further confirms that the vertical extent of the strong wave perturbation is gradually suppressed as $c / u_{* s}$ decreases. The pressure stress $\left\langle\tau^{p}\right\rangle$ continues to increase near the surface, approaching almost $70 \%$ of the wind stress for $c / u_{* s}=-11$.

The two-dimensional fields of $\overline{\tau^{t}}$ and $\overline{\tau^{w}}$ for waves opposing wind (Figure 2.5d$\mathrm{f}, 5 \mathrm{j}-\mathrm{l})$ also show that the stress field for $c / u_{* s}=-1.4$ remains qualitatively similar to $c / u_{* s}=1.4$, and this pattern remains largely unchanged up to $c / u_{* s}=-11$ with all wave perturbations slowly weakening and migrating down as $c / u_{* s}$ decreases. One notable development is the emergence of a region of positive $\overline{\tau^{t}}=\overline{u^{\prime} W^{\prime}}$ just 
above the trough at $c / u_{* s}=-5.6$ and -11 , where the flow perturbation of enhanced negative $\omega_{y}$ (wind shear) and reduced TKE have been observed earlier.

\section{Energy budget and turbulence closure parameterization}

We next investigate the energy budget inside the wave boundary layer. Hara and Sullivan [51] have derived the equations governing the wave-fluctuation energy, $E^{w}=(1 / 2)(\tilde{u} \tilde{u}+\tilde{w} \tilde{w})$, and the turbulent kinetic energy, $\bar{e}=(1 / 2)\left(\overline{u^{\prime} u^{\prime}}+\overline{v^{\prime} v^{\prime}}+\overline{w^{\prime} w^{\prime}}\right)$, in mapped coordinates. If the two governing equations are combined, averaged horizontally and normalized, the result yields

$$
\left\langle\tilde{u} \frac{1}{J}\right\rangle \frac{\partial P}{\partial x} \frac{\kappa \zeta}{u_{* s}^{3}}+\frac{\partial\langle u\rangle}{\partial \zeta} \frac{\kappa \zeta}{u_{* s}}-\frac{\partial\left(F^{w}+F^{t}\right)}{\partial \zeta} \frac{\kappa \zeta}{u_{* s}^{3}}-\left\langle\frac{1}{J} \epsilon\right\rangle \frac{\kappa \zeta}{u_{* s}^{3}}=0
$$

where the second term is the shear production term, the third term is the transport term ( $F^{w}$ and $F^{t}$ are the vertical transport of $E^{w}$ and $\bar{e}$, respectively), and the fourth term is the viscous dissipation term. The first term arises because of the imposed pressure gradient (i.e., because the stress is not constant in vertical). Here, $\left(F^{w}+F^{t}\right)$ at the surface is equal to the energy flux into the waves. Refer to Hara

and Sullivan [51] for more details on the derivation of the energy budget in mapped coordinates.

In Figure 2.6a,b, the second, third and fourth terms of Equation 3.3 are plotted in solid, dot-dashed, and dotted lines with colors corresponding to their respective $c / u_{* s}$. The first term is approximately zero and shown as a solid grey line. Thin dotted lines near zero are equal to the sum of all the energy budget terms. Thin grey lines represent the mean shear production (mean wind shear) over a flat wall for linearly decreasing stress (solid) and constant stress (dashed) with respect to 
$k \zeta$. Note that the energy budget is not fully closed in the case of $c / u_{* s}=-11$. We suspect that this error arises in the calculation of the transport term and is caused by the increasingly strong pressure signal, but that the shear production and the dissipation terms are still reasonably accurate.

Previous modeling studies of the vertical mean wind profile and the drag coefficient over a surface wave train (or a spectrum of waves, i.e., many surface wave trains superimposed) have sought to close the turbulence in the wave boundary layer by parameterizing the eddy viscosity $(K)$ or the TKE dissipation rate $(\langle\epsilon / J\rangle)$ using the turbulent stress $\left\langle\tau^{t}\right\rangle$ that varies with height due to the wave influence (e.g. Hara and Belcher 50, Makin and Kudryavtsev 66). Thus one area of interest in the present study is to determine whether the character of the mean normalized wind shear (equivalent to the mean shear production term of the energy budget, solid lines in Figure 2.6a,b), the mean dissipation (last term in Equation 3.3, dotted lines in Figure 2.6a,b and identical to Figures 2.4c,d), and the mean turbulent stress share similarities in character over a range of wind-wave conditions. For this reason, we have included the profiles of the mean turbulent stress in Figure 2.6c,d (previously shown in Figures 5C and 5D, respectively), and the profiles of the normalized eddy viscosity $K /\left(\kappa u_{* s} \zeta\right)$ in Figure 2.6e,f, where $K=\left\langle\tau^{t}\right\rangle /(\partial\langle u\rangle / \partial \zeta)$.

In all cases, the shear production roughly balances the viscous dissipation throughout the wave boundary layer, with a relatively modest contribution from the transport term (except for $c / u_{* s}=-11$ ). The profiles of the shear production and viscous dissipation are reasonably correlated with the profile of the turbulent stress (Figure 2.6c,d). All three profiles are significantly reduced near the surface for all $c / u_{* s}$ cases, and enhanced further above in the cases of $c / u_{* s}=1.4,-1.4,-5.6$, -11 , although the enhancement of $\left\langle\tau^{t}\right\rangle$ occurs somewhat above the enhancement 
of the shear production and the viscous dissipation in the cases of $c / u_{* s}=-5.6$ and -11 . This suggests that the existing turbulent closure model to parameterize $\epsilon$ in terms of $\left\langle\tau^{t}\right\rangle$ may be appropriate for a wide range of $c / u_{* s}$.

One notable exception to the generally good correlation between the dissipation rate and the turbulent stress is that for the cases of waves opposing wind, the shear production and viscous dissipation are both significantly reduced (by as much as $1 / 2)$ above $k \zeta=1$ all the way to the top $(k \zeta=4)$, even if the turbulent stress $\left\langle\tau^{t}\right\rangle$ is

almost equal to the total wind stress $\left\langle\tau^{t o t}\right\rangle$ (that is, the wave effect on the turbulent stress is negligible) in the same $k \zeta$ range. As discussed earlier, this reduced mean wind shear makes a significant contribution to the enhancement of the equivalent roughness length $z_{o}$ and the drag coefficient. A turbulence closure model based on the wave modified $\left\langle\tau^{t}\right\rangle$ alone would completely miss this impact.

The profiles of the normalized eddy viscosity $K /\left(\kappa u_{* s} \zeta\right)$ for waves following wind (Figure 2.6e) show that they are not too far from the grey solid line throughout the wave boundary layer, but its variation is not well correlated with $\left\langle\tau^{t}\right\rangle$. For waves opposing wind, the normalized eddy viscosity significantly increases roughly above $k \zeta=0.4$, and this increase is totally uncorrelated with $\left\langle\tau^{t}\right\rangle$. These observations suggest that parameterizing $K$ inside the wave boundary layer in terms of $\left\langle\tau^{t}\right\rangle$ is more problematic for waves opposing wind.

\section{Wave growth/decay rate and equivalent roughness length}

The normalized phase-dependent surface stress distribution is plotted in Figure 2.1A,B for waves following wind, and in Figure 2.1C,D for waves opposing wind. Since the total normal stress is a sum of the pressure and the turbulent normal stress, both the total normal stress (solid line) and the pressure stress alone 
(dotted line) are shown in Figure 2.1A,C. The turbulent tangential surface stress is presented in Figure 2.1B,D.

Both the normal stress in phase with the normal wave orbital velocity and the tangential stress in phase with the tangential wave orbital velocity contribute to the total energy flux $(E F)$ into waves. The wave growth/decay rate $\beta$ (which is positive/negative for wave growth/decay) is then calculated by dividing $E F$ by the wave energy. We use the common expression of the wave growth/decay rate

$$
\beta=c_{\beta}\left(\frac{u_{* s}}{c}\right)^{2} \frac{\rho_{a}}{\rho_{w}} \omega
$$

where $\omega$ is the wave frequency. The coefficient $c_{\beta}$ is then evaluated based on the total energy flux $\left(c_{\beta t o t}\right)$ as well as with the tangential turbulent stress only $\left(c_{\beta t}\right)$, with the normal turbulent stress only $\left(c_{\beta n}\right)$ and with the pressure only $\left(c_{\beta p}\right)$. The results are summarized in Table 2.1 and in Figure 2.7a. For more details on how we compute each component of $c_{\beta}$, refer to Hara and Sullivan [51] and Husain et al. [56].

Consistent with Husain et al. [56] for waves with $c / u_{* s}=1.4$, the pressure field (as well as the total normal stress field) is strongly modified by wind flow over steep, strongly forced waves. In the "dead zone" above the leeward face of the wave (where wind velocity is weak), pressure remains almost zero before increasing to a maximum downstream of the trough on the windward face of the wave. The significant downstream phase shift of the pressure maximum from the trough is responsible for the large energy flux and a resulting $c_{\beta t o t}$ of 18.0. The addition of a nonlinear Stokes solution to the bottom wave shape slightly increases $c_{\beta t o t}$ to 18.2 (see black squares in Figure 2.7a). With increasing $c / u_{* s}$, the magnitude of the 
pressure (and the total normal stress) variation and the resulting energy flux to waves rapidly decrease, and consequently $c_{\beta t o t}$ decreases to 3.9 at $c / u_{* s}=11$ (see thick solid black line in Figure 2.7a).

For waves opposing wind, as $c / u_{* s}$ becomes more negative the pressure perturbation becomes stronger and more in phase with $-h$ (negative wave elevation) due to the increasing relative wind velocity $(\langle u>-c)$, as discussed in Subsection a (note the difference in vertical scale between Figure 2.1A,C). Nevertheless, a significant pressure component out of phase with $-h$ exists (buried in the strong in phase component) and its contribution to the negative energy flux and the wave decay rate significantly increases with increasingly negative $c / u_{* s}$.

At $c / u_{* s}=-1.4$ the magnitude of $c_{\beta t o t}=-17.2$ is close to the magnitude of $c_{\beta t o t}=18.0$ at $c / u_{* s}=1.4$. Therefore, while the direction of energy flux reverses between these two cases, its magnitude is similar. The magnitude of $c_{\beta p}$, which is proportional to the pressure form drag, is also similar between the two cases. Recall that the momentum flux is downward in both cases even if the energy flux is downward/upward with waves following/opposing wind. As wave speed increases and $c / u_{* s}$ decreases to $-11, c_{\beta t o t}$ significantly decreases to -24.9 (Figure 2.7a).

In Figure 2.8, our estimated $c_{\beta t o t}$ and $c_{\beta p}$ values (Figure 2.8a) and $\beta / \omega$ values (Figure 2.8b,c) for waves opposing wind are compared to the previous LES study of Cao et al. [16] as well as the results of Harris et al. [52], Cohen [20], Donelan [25], Peirson et al. [80], and Mitsuyasu and Yoshida [70]. Since most previous studies focused on wave attenuation due to pressure only, they should be compared with our results for $c_{\beta p}$ or corresponding $\beta / \omega$ (thin red lines).

Our results, plotted against $\left|c / u_{* s}\right|$ or $\left|u_{* s} / c\right|$, are near the lower end of the other studies (Figure 2.8a,b). In particular, the results of Cao et al. [16] (the 
only other LES study) are consistently larger than ours by a factor of around 2. The differences we do see may be due to our choice of a rather steep wave slope $a k=0.27$ compared to $a k=0.08-0.15$ used in Cao et al. [16]. Such difference is not surprising since it has been well accepted that the growth rate of waves following wind may vary significantly depending on the wave slope and other wind and wave characteristics. When we plot the decay rate as a function of $\left(\langle u\rangle_{\lambda / 2} / c-1\right)\left|\langle u\rangle_{\lambda / 2} / c-1\right|$ (Figure 2.8c), our results appear to be closer to those of Cao et al. [16] (see their Figure 19) and are quite consistent with the observations and the parameterization of Donelan [25] (see their Figure 4).

In Figure 2.7b we summarize the estimated values for $z_{o} / z_{o b}$, which represents the enhancement of the effective roughness length $z_{o}$ due to resolved waves relative to the background roughness length $z_{o b}$. The results of $z_{o} / z_{o b}$ are shown in a log scale because the increasing wind speed above the wave boundary layer due to resolved waves is proportional to $\log \left(z_{o} / z_{o b}\right)$. The figure highlights the strong dependence of the effective roughness length and the drag coefficient on $c / u_{* s}$. With waves following wind they rapidly decrease as $c / u_{* s}$ increases. However, with waves opposing wind, they rapidly increase with increasingly negative $c / u_{* s}$, that is, faster waves opposing wind have the largest impact on the drag coefficient.

It is interesting that while the magnitude of $c_{\beta p}$ is similar (i.e., the pressure form drag is similar) between the cases of $c / u_{* s}=1.4$ and -1.4 , the wave enhanced effective roughness length $z_{o} / z_{o b}$ is significantly larger for waves opposing wind. This suggests that the increase in the effective roughness (drag coefficient) is not necessarily caused by an increase in the wave form drag. This difference in behavior of $c_{\beta p}$ and $z_{o} / z_{o b}$ can be explained based on the energy budget inside the wave boundary layer. 
Hara and Belcher [50] show that inside the constant stress layer the downward energy flux at the top of the wave boundary layer is roughly equal to the mean wind speed multiplied by the wind stress $\left(\bar{u} \tau_{s}\right)$ because the vertical TKE transport is small there, and that this energy input is balanced by the viscous dissipation integrated over the entire wave boundary layer plus the energy flux into surface waves at the bottom. Therefore, with a fixed wind stress (as in the current study), as the viscous dissipation inside the wave boundary layer increases, or the energy input to surface waves increases, the wind speed at the top increases and the drag coefficient and effective surface roughness decrease.

Hara and Belcher [50] then assume that the reduction of the viscous dissipation inside the wave boundary layer is correlated with the reduction of the turbulent stress, which is mostly due to the pressure form drag. Therefore, if the pressure form drag is similar between the cases of $c / u_{* s}=1.4$ and $c / u_{* s}=-1.4$, we would expect that an overall reduction of the viscous dissipation and the resulting decrease of the wind speed at the top of the wave boundary layer are similar as well. However, the energy flux into surface waves is positive (negative) for waves following (opposing) wind (although its magnitude is similar), and consequently the wind speed near the top of the wave boundary layer increases (decreases) and the drag coefficient and effective roughness length decrease (increase). This reversal of the energy flux direction to/from waves may explain why $z_{o} / z_{o b}$ is significantly larger for waves opposing wind. In fact, a quick estimate of the difference in the far field wind speed $\langle u\rangle / u_{* s}$ due to the reversal of the energy flux alone (estimate made using the $c_{\beta t o t}$ values in Table 2.1) is about 1.3, which is roughly consistent with the observed difference of 1.7 at $k \zeta=4$ (roughly the height of the wave boundary layer) in our LES results. This indicates that the increase of the equivalent roughness from 
$c / u_{* s}=1.4$ to $c / u_{* s}=-1.4$ is mainly attributed to the reversal of the energy flux. Our LES results therefore suggest that it is important to account for the effect of energy flux to/from surface waves (in addition to the wave form drag) when estimating the wave modified effective roughness length $z_{o}$ and the drag coefficient, particularly when waves oppose the wind.

\subsection{Summary}

In this study, we use large eddy simulation (LES) to simulate turbulent wind flow over steep waves $(a k=0.27)$ following and opposing the wind for a range of wave speeds relative to wind forcing $\left(\left|c / u_{* s}\right|=1.4,2.8,5.6,8.2\right.$, and 11). Our results show that the phase averaged wind flow patterns over slow waves following wind $\left(c / u_{* s}=1.4\right)$ are strongly influenced by intermittent but frequent airflow separations, characterized by enhanced spanwise vorticity detached from the leeward crest. The winds weaken and recirculate in the trough of the wave. Inside the dead zone below the detached enhanced vorticity layer, the TKE, viscous dissipation, and vorticity are all significantly reduced (Figure 2.1a,g,m,s and 2a,g,m,A,G,M). Flow separation alters the mean wind profile (Figure 2.4) and induces shifts in the pressure field such that the wind stress partition (including form drag, Figure 2.5) and wave growth rate (Figure 2.7) are modified significantly. As wave age increases, the frequency of intermittent airflow separation events and their signature in the phase averaged flow fields rapidly diminishes.

Wind flow over opposing waves results in a strong wave-induced flow perturbation that intensifies and is compressed near the surface as the phase speed of the waves increases. We observe a number of phase-averaged flow features similar to those over slow waves following wind, i.e., enhanced TKE, dissipation, and detached vorticity 
near the wave crest, as well as reduced TKE, dissipation, and vorticity in the wave trough below the detached enhanced vorticity layer (Figure 2.1D-F,J-L,P-R). However, the strong positive wind along the wave shape over opposing waves (in a frame of reference moving with the wave) inhibits apparent separation-like flow patterns. Increases in opposing wave speed intensify the in-phase component of the pressure field (Figure 2.1v-x,C) and make the flow appear to follow the potential wave theory. They also induce a significant out-of-phase component of the pressure field responsible for an increase in the effective surface roughness and wave decay rate (Figure 2.7). Our estimated wave decay rates are consistent with those of previous studies, including a recent study using wall-resolved LES (Cao et al. 16), model studies using RANS solutions (Al-Zanaidi and Hui 1, Cohen 20, Harris et al. 52, Mastenbroek 68), and laboratory studies (Donelan 25, Mitsuyasu and Yoshida 70, Peirson et al. 80).

It is noteworthy that the observed separation-like signatures over opposing wind are qualitatively similar to flow separations over rotating cylinder discussed by Gad-el Hak and Bushnell [40] and Degani et al. [22]. Gad-el Hak and Bushnell [40] notes that there is a close relationship between steady flow over a moving wall and unsteady flow over a fixed wall, and that in these conditions separation points may be lifted above the surface and the traditional criterion of zero surface shear stress does not apply. In fact, they predict that near surface wind shear becomes negative/positive over a cylinder rotating forward (backward), which corresponds to a crest of waves opposing (following) wind. This prediction is consistent with our LES results discussed earlier, in particular, producing strong negative vorticity in the trough for faster waves (Figure 2.2P,Q,R; also see Figure 1 in Degani et al. 22). 
Our estimates of the equivalent surface roughness $z_{o}$ (including the effect of resolved waves) relative to the background roughness $z_{o b}$ (representing the form drag of unresolved waves and viscosity) show that the enhancement $z_{o} / z_{o b}$ is significant for slow waves following wind but it decreases as the wave age increases. On the other hand, for waves opposing wind, $z_{o} / z_{o b}$ rapidly increases as the wave speed increases. By comparing the results of the slowest waves for both following and opposing wind $\left(c / u_{* s}=1.4\right.$ and -1.4$)$, we find that the pressure form drag is very similar but $z_{o} / z_{o b}$ is significantly larger with waves opposing wind, suggesting that the increase of equivalent roughness length (or drag coefficient) is not necessarily caused by an increase of the wave form drag. We refer to the study of Hara and Belcher [50] and speculate that the reversal of energy flux direction from wind to waves (from waves to wind) for waves following (opposing) wind is responsible for the difference in $z_{o} / z_{o b}$. This finding suggests that it is important to account for the reversed energy flux when estimating the enhancement of the effective surface roughness due to opposing waves.

Waves opposing wind often appear when the wind field rapidly changes in space and/or time; a situation commonly encountered under tropical cyclones. Previous modeling efforts of the sea-state dependent drag coefficient have predicted waves opposing wind may significantly enhance the drag coefficient in such conditions because of the assumed large form drag (Chen and Curcic 18, Chen et al. 19, Reichl et al. 83). Results from the present study provide credible support for such modeling efforts. In addition, our energy budget analysis (Section 3f) identifies the strengths and weaknesses of existing models of mean wind profile and drag coefficient over a spectrum of waves. 


\subsection{Acknowledgements}

We acknowledge support of the National Science Foundation (Physical Oceanography) Grant OCE-1458984 (URI). We also acknowledge high-performance computing support from Yellowstone and Cheyenne (doi:10.5065/D6RX99HX) provided by NCAR's Computational and Information Systems Laboratory, sponsored by the National Science Foundation.

\subsection{Figures and Tables}


Table 2.1: List of run conditions and results of roughness enhancement $z_{o} / z_{o b}$ and non-dimensional wave growth/decay coefficient $c_{\beta}$ for eleven LES simulations. The symbol $\mathrm{f}$ and $\mathrm{o}$ in the run name represents waves following and opposing wind, respectively. The symbol s in the run name represents Stoke waves. Nondimensional parameters used in LES cases include wave age $\left(c / u_{* s}\right.$, which is negative for wind opposing wind), wave steepness $(a k)$, and parameterized background roughness length $\left(k z_{o b}\right)$. Wave steepness is held constant for all simulations at $a k=0.2665(a k \sim 0.27)$. Parameterized background roughness is also held constant at $k z_{o b}=2.70 \times 10^{-3}$. The values for $c_{\beta t}, c_{\beta n}$, and $c_{\beta p}$ refer to tangential turbulent stress, normal turbulent stress, and pressure contributions to $c_{\beta}$, and $c_{\beta t o t}$ is the total.

$\begin{array}{lcccccc}\text { Run } & c / u_{* s} & z_{o} / z_{o b} & c_{\beta t} & c_{\beta n} & c_{\beta p} & c_{\beta t o t} \\ \text { 1.4f } & 1.4 & 4.65 & 2.8 & 1.6 & 13.6 & 18.0 \\ \text { 1.4fs } & 1.4 & 5.82 & 2.2 & 1.3 & 14.8 & 18.2 \\ \text { 1.4o } & -1.4 & 7.04 & -3.5 & -0.5 & -13.1 & -17.2 \\ \text { 2.8f } & 2.8 & 3.96 & 2.0 & 1.8 & 13.9 & 17.7 \\ \text { 2.8o } & -2.8 & 8.69 & -3.9 & 0.0 & -13.2 & -17.1 \\ \text { 5.6f } & 5.6 & 4.09 & 0.1 & 1.3 & 13.8 & 15.2 \\ \text { 5.6o } & -5.6 & 14.92 & -4.8 & 1.1 & -14.6 & -18.3 \\ \text { 8.2f } & 8.2 & 2.97 & -1.3 & 0.6 & 10.9 & 10.3 \\ \text { 8.2o } & -8.2 & 20.31 & -5.3 & 1.8 & -16.9 & -20.9 \\ \text { 11.0f } & 11.0 & 1.58 & -2.5 & -0.1 & 6.6 & 3.9 \\ 11.0 \mathrm{o} & -11.0 & 31.80 & -7.1 & 2.1 & -19.8 & -24.9\end{array}$



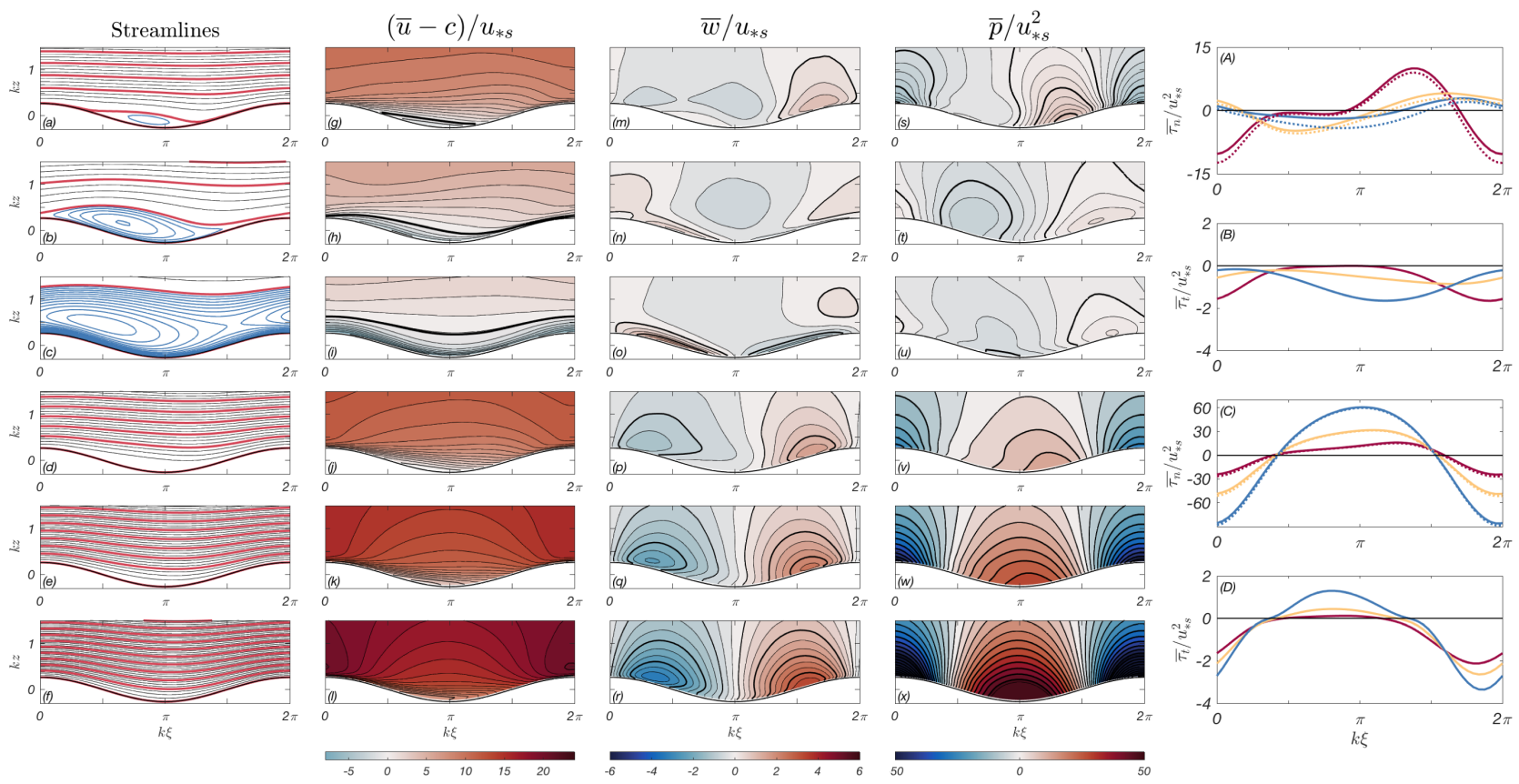

Figure 2.1: Normalized phase-averaged flow fields in the $\xi$ - $z$ coordinate for waves following wind $\left(c / u_{* s}=1.4,5.6,11.0\right.$; top three panels $)$ and waves opposing wind $\left(c / u_{* s}=-1.4,-5.6,-11.0\right.$; bottom three panels $)$ from left to right: streamlines, horizontal velocity $\left((\bar{u}-c) / u_{* s}\right)$, vertical velocity $\left(w / u_{* s}\right)$, and pressure $\left(\bar{p} / u_{* s}^{2}\right)$. Rightmost plots show the surface stress distribution for the normal stress $\overline{\tau_{n}} / u_{* s}^{2}$ (panels $\mathrm{A}$ and $\mathrm{C}$, solid line is total normal stress and dotted line is pressure only) and for the tangential stress $\overline{\tau_{t}} / u_{* s}^{2}$ (panels B and D) for $\left|c / u_{* s}\right|=1.4,5.6$, and 11.0 (dark red, light orange, blue). The bottom two panels are for waves opposing wind. 

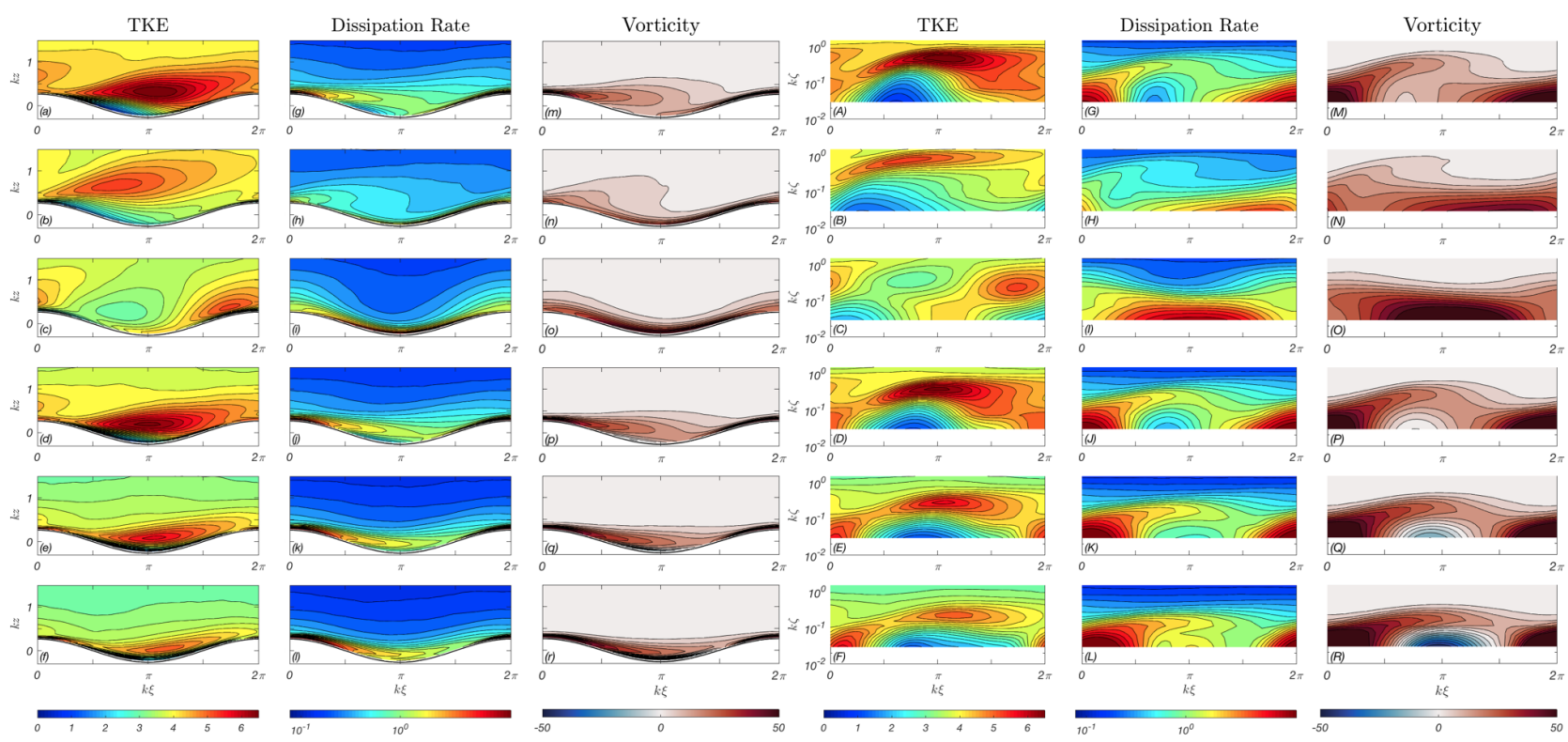

Figure 2.2: Normalized phase-averaged flow fields in the $\xi-z$ coordinate (left three panels) and the mapped $\xi-\zeta$ coordinate (right three panels) for waves following wind $\left(c / u_{* s}=1.4,5.6,11.0\right.$; top three panels $)$ and waves opposing wind $\left(c / u_{* s}=\right.$ $-1.4,-5.6,-11.0$; bottom three panels) from left to right: turbulent kinetic energy $\left(\bar{e} / u_{* s}^{2}\right)$, dissipation rate $\left(\epsilon /\left(k u_{* s}^{3}\right)\right)$, and spanwise vorticity $\left(\bar{\omega}_{y} /\left(k u_{* s}\right)\right)$. 

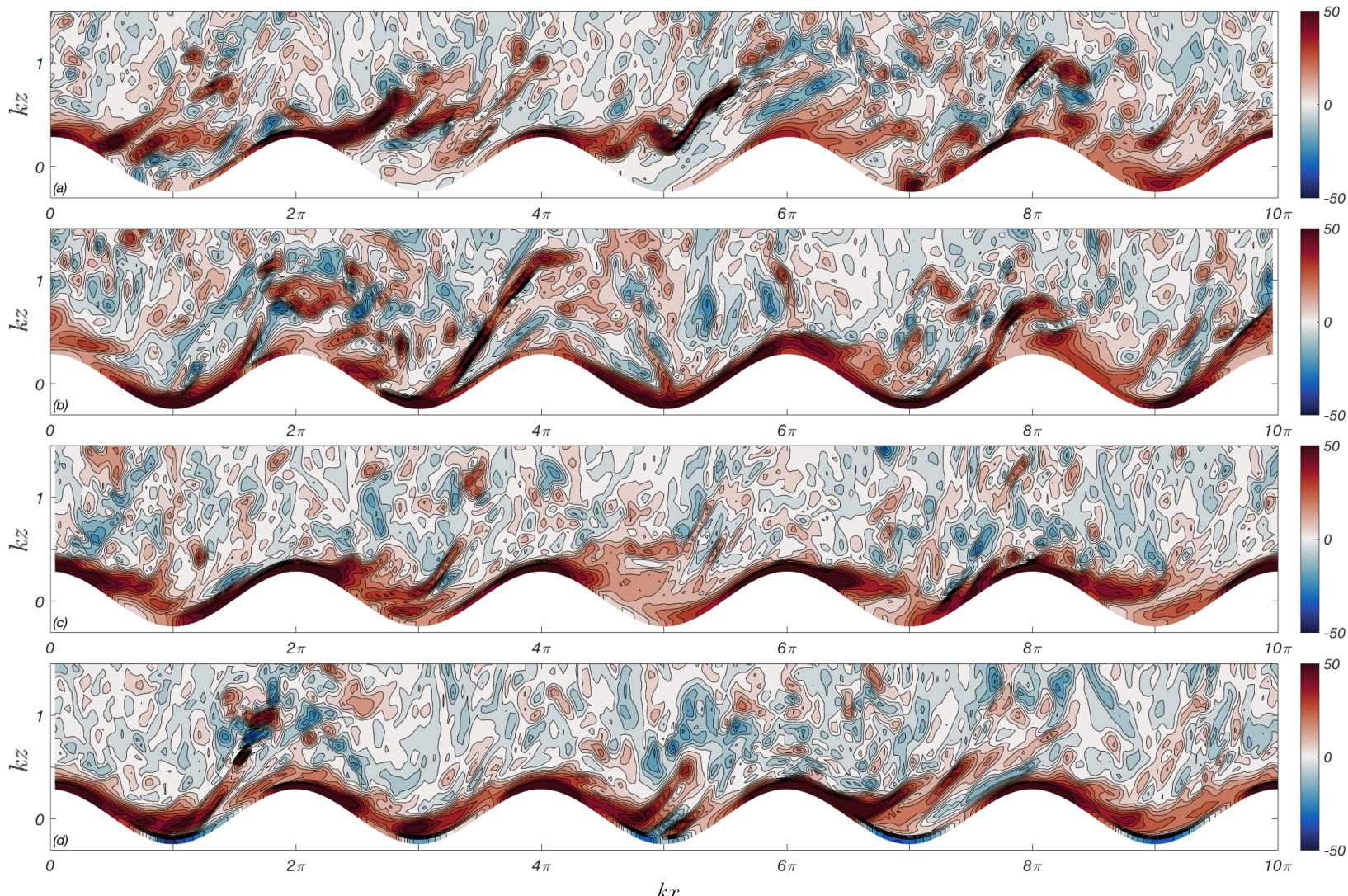

Figure 2.3: Normalized instantaneous vorticity fields $\left(\omega_{y} /\left(k u_{* s}\right)\right)$ in the $x-z$ coordinate for waves following wind $\left(c / u_{* s}=1.4,11.0\right.$; top two panels $)$ and waves opposing wind $\left(c / u_{* s}=-1.4,-11.0\right.$; bottom two panels). 

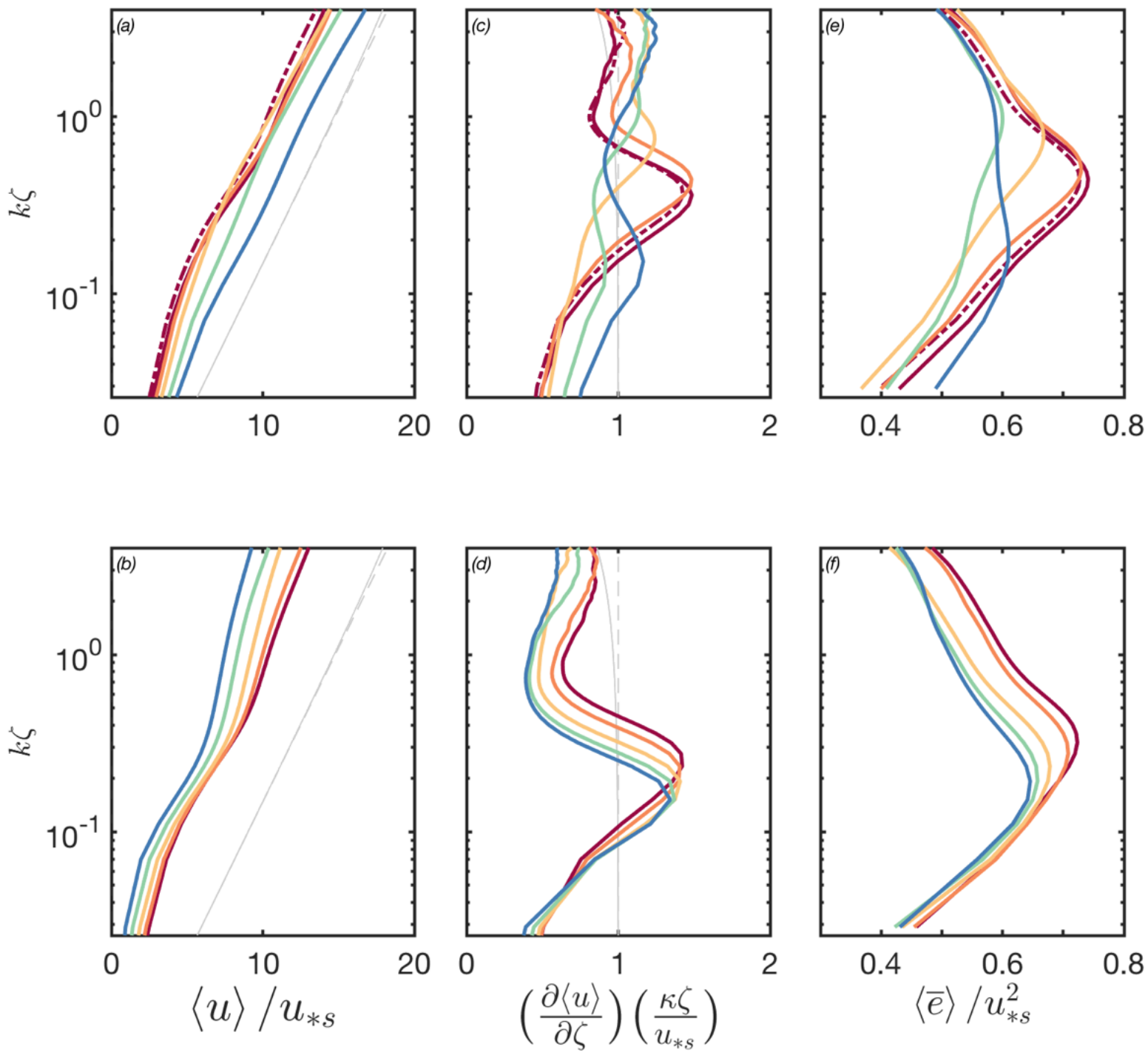

Figure 2.4: Normalized vertical profiles of horizontally averaged wind speed $\left(\langle u\rangle / u_{* s}^{2}\right)$, wind shear $\left(\frac{\partial\langle u\rangle}{\partial \zeta} \frac{\kappa \zeta}{u_{* s}}\right)$, and TKE $\left(\langle\bar{e}\rangle / u_{* s}^{2}\right)$ for waves following wind (top panel) and waves opposing wind (bottom panel) for $\left|c / u_{* s}\right|=1.4,2.8,5.6,8.2$, and 11.0 (dark red, dark orange, light orange, light green, and blue). The dot-dashed dark red line is the Stokes wave case for $c / u_{* s}=1.4$. 

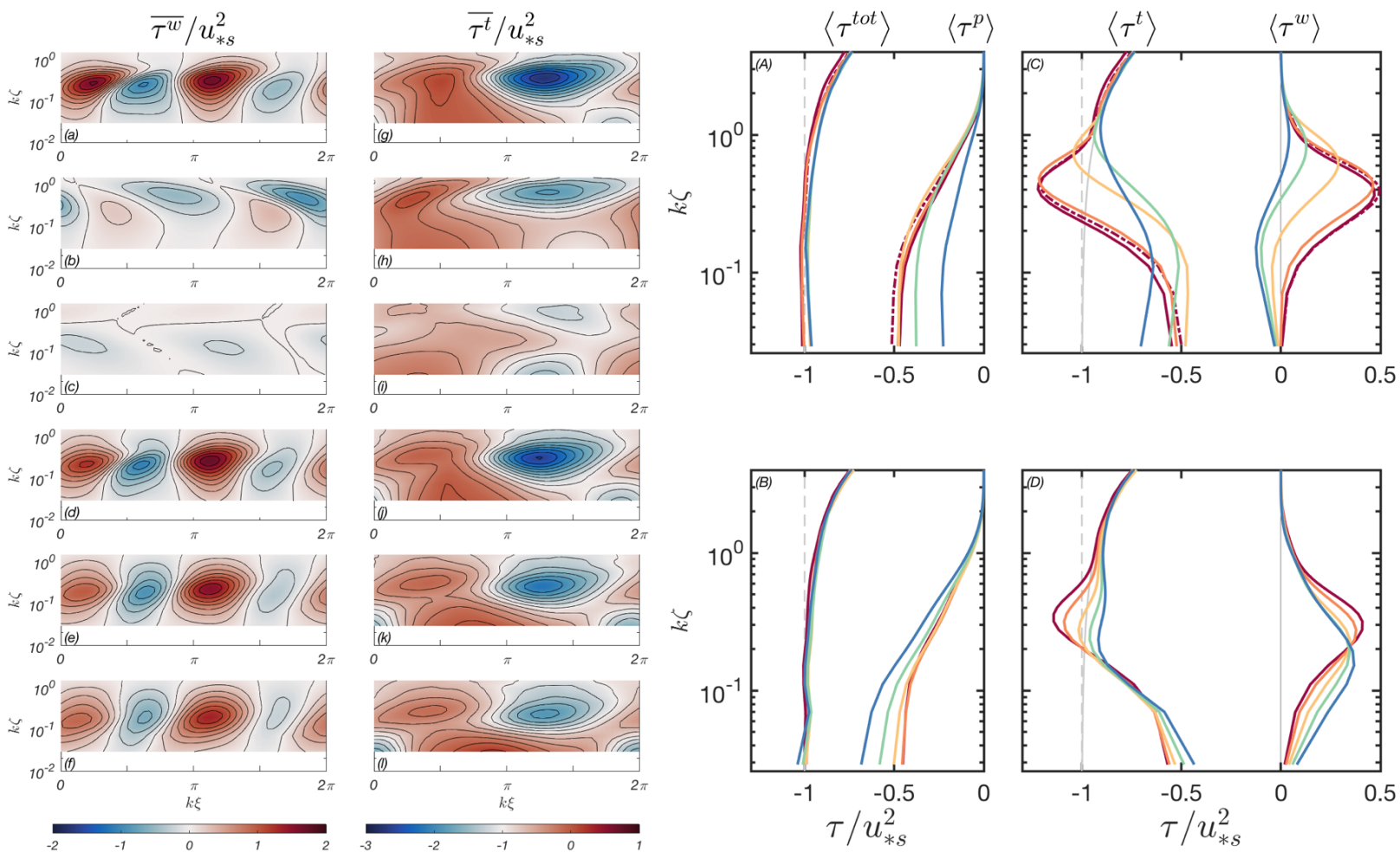

Figure 2.5: On the left: normalized phase-averaged fields of wave-coherent stress $\left(\overline{\tau^{w}} / u_{* s}^{2}=\tilde{u} \tilde{W} / u_{* s}^{2}\right)$ and turbulent stress $\left(\overline{\tau^{t}} / u_{* s}^{2}=\overline{u^{\prime} W^{\prime}} / u_{* s}^{2}\right)$ in the mapped $\xi-\zeta$ coordinate for waves following wind $\left(c / u_{* s}=1.4,5.6,11.0\right.$; top three panels $)$ and waves opposing wind $\left(c / u_{* s}=-1.4,-5.6,-11.0\right.$; bottom three panels $)$. On the right: normalized vertical profiles of horizontally averaged momentum budget terms for waves following wind (top panels) and waves opposing wind (bottom panels), including the total wind stress $\left(\left\langle\tau^{t o t}\right\rangle / u_{* s}^{2}\right)$, pressure stress $\left(\left\langle\tau^{p}\right\rangle / u_{* s}^{2}\right)$, turbulent stress $\left(\left\langle\tau^{t}\right\rangle / u_{* s}^{2}\right)$, and wave-coherent stress $\left(\left\langle\tau^{w}\right\rangle / u_{* s}^{2}\right)$ for $\left|c / u_{* s}\right|=1.4,2.8,5.6$, 8.2, and 11.0 (dark red, dark orange, light orange, light green, and blue). The dot-dashed dark red line is the Stokes wave case for $c / u_{* s}=1.4$. 

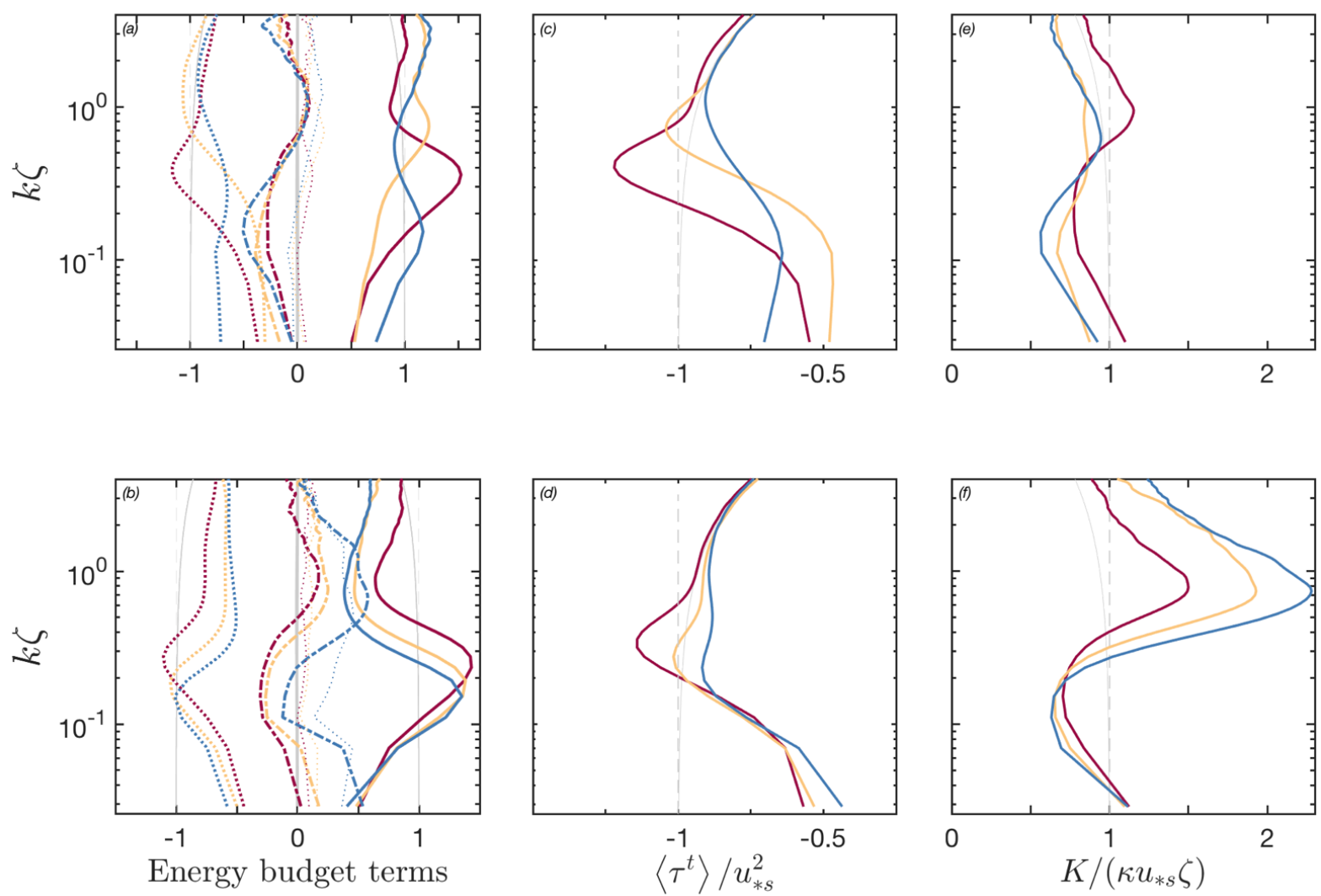

Figure 2.6: Left plots: normalized vertical profiles of horizontally averaged energy budget terms (the first, second, third and fourth terms of Equation 3.3 are solid grey, solid, dot-dashed, and dotted lines, respectively) with thin dotted lines near zero equalling the sum of all energy budget terms. Middle plots: normalized vertical profiles of horizontally averaged turbulent stress $\left(\left\langle\tau^{t}\right\rangle / u_{* s}^{2}\right.$, also shown in Figure 2.5). Right plots: normalized vertical profiles of eddy viscosity $\left(K /\left(\kappa u_{* s} \zeta\right)\right)$. For the left, middle, and right plots, the thin grey lines represent mean shear production, turbulent stress, and eddy viscosity profiles over a flat wall with linearly decreasing wind stress (solid) and constant stress (dashed) with respect to $k \zeta$. Results for waves following (opposing) wind are shown on the top (bottom) panel for $\left|c / u_{* s}\right|=$ 1.4, 5.6, and 11.0 (dark red, light orange, and blue). 

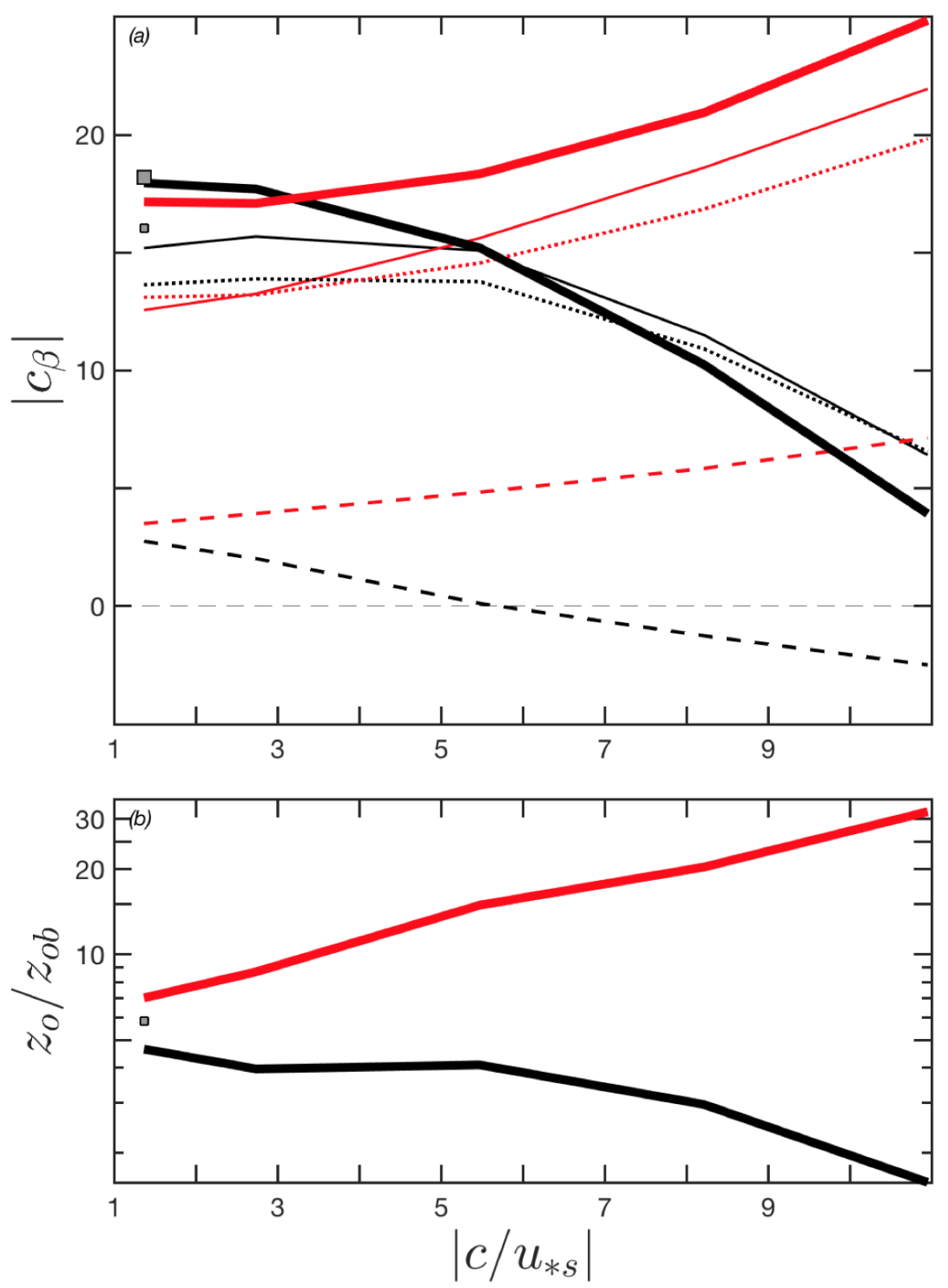

Figure 2.7: Top plot: wave growth/decay coefficient $\left|c_{\beta}\right|$ for waves following wind (black lines) and for waves opposing wind (red lines) as a function of $\left|c / u_{* s}\right|$. Thin solid lines represent the contribution of the total normal stress $\left(c_{\beta p}+c_{\beta n}\right)$, dotted lines represent the pressure contribution $\left(c_{\beta p}\right)$, dashed lines represent tangential stress contribution $\left(c_{\beta t}\right)$, and thick solid lines represent the sum of all components $\left(c_{\beta t o t}=c_{\beta p}+c_{\beta n}+c_{\beta t}\right)$. Bottom plot: ratio of the equivalent surface roughness to the background (parameterized) surface roughness $z_{o} / z_{o b}$ as a function of $\left|c / u_{* s}\right|$ for waves following wind (black line) and waves opposing wind (red line). In both plots, the dark grey squares represent the Stokes wave case for $c / u_{* s}=1.4$ for $c_{\beta t o t}$ (large square) and $c_{\beta p}$ only (small square). 

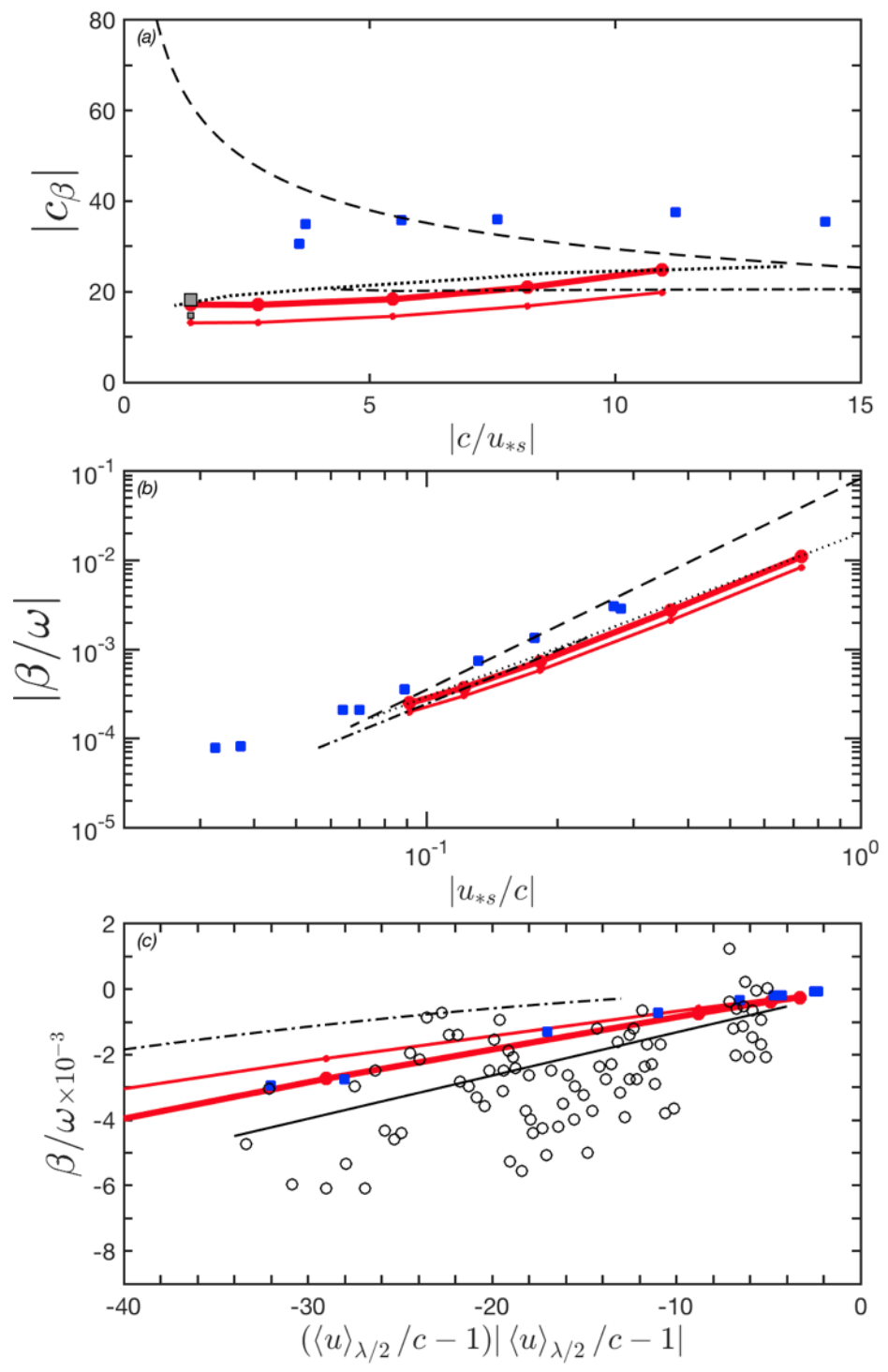

Figure 2.8: Top plot: Comparison of wave decay coefficient $\left|c_{\beta}\right|$ as a function of wave age $\left|c / u_{* s}\right|$ for the current LES study (thick red line: total $c_{\beta t o t}$, thin red line: only $c_{\beta p}$ ), the wall-resolved LES results of Cao et al. [16] (blue squares), the parameterization of Mitsuyasu and Yoshida [70] (dashed line), and the results of Harris et al. [52] (dot-dashed line) and Cohen [20] (dotted line). Middle plot: Comparison of wave decay rate $|\beta / \omega|$ as a function of inverse wave age $\left|u_{* s} / c\right|$ with lines corresponding to those of the top plot. Compare with Cao et al. [16] Figure 18. Bottom plot: Comparison of wave decay rate $\beta / \omega$ as a function of $\left(\left\langle u_{\lambda / 2}\right\rangle / c-1\right)\left|\left\langle u_{\lambda / 2}\right\rangle / c-1\right|$, including the current LES results (thick red line: total decay rate, thin red line: pressure contribution only), the wall-resolved LES of Cao et al. [16] (blue squares), the parameterization of Mitsuyasu and Yoshida [70] (dot-dashed line), and the observational results and parameterization of Donelan [25] (black circles and black line, respectively). Compare to Figure 19 of Cao et al. [16]. 


\title{
Wind turbulence over misaligned surface waves and air-sea momentum flux. Part II: Waves propagating oblique to wind
}

\author{
Nyla Husain ${ }^{1 *}$, Tetsu Hara ${ }^{1}$, and Peter P. Sullivan ${ }^{2}$
}

Prepared for submission to Journal of Physical Oceanography

\footnotetext{
${ }^{1}$ Graduate School of Oceanography, University of Rhode Island, Narragansett, Rhode Island ${ }^{*}$ Corresponding author email: nylahusain@uri.edu

${ }^{2}$ National Center for Atmospheric Research, Boulder, Colorado
} 


\subsection{Abstract}

The coupled dynamics of turbulent airflow and a spectrum of waves are known to modify air-sea momentum and scalar fluxes. Waves traveling at oblique angles to the wind are common in the open ocean, and their effects may be especially relevant when constraining fluxes in storm and tropical cyclone conditions. In this study, we employ large eddy simulation for airflow over steep, strongly forced waves following and opposing oblique wind to elucidate its impacts on the wind speed magnitude and direction, drag coefficient, and wave growth/decay rate. We find that oblique wind maintains a signature of airflow separation while introducing a cross-wave component strongly modified by the waves. The directions of mean wind speed and mean wind shear vary significantly with height and are misaligned from the wind stress direction particularly toward the surface. While waves oblique to wind support significant wave form drag, they appear to have little impact on the equivalent surface roughness and the drag coefficient. Therefore, our findings have significant implications for how the sea-state dependent drag coefficient is parameterized in forecast models. 


\subsection{Introduction}

Coupling between the ocean and atmosphere is driven by turbulent air-sea fluxes of momentum, energy, heat, and gases, and it influences the evolution of marine weather and climate patterns. Constraining these air-sea fluxes in numerical models continues to be a challenge due to our incomplete understanding of near-surface air/water turbulence that is strongly modified by surface wave processes (Cronin et al. 21).

Previous studies have addressed the impacts of surface waves on the wind profile and drag coefficient $C_{d}$ (e.g. Donelan et al. 28, Fan et al. 39, Moon et al. $73,74,75$, Reichl et al. 83), often focusing on key wave parameters such as the wave age, wave curvature, and wave slope (e.g., Banner 4, Belcher et al. 8, Donelan 26, Donelan et al. 27, 30, Edson et al. 36, Makin and Kudryavtsev 66). In several modeling studies, sea-state dependent $C_{d}$ parameterizations based on full surface wave spectra have been developed to account for the effects of complex sea states in high to extreme winds - including conditions in which $C_{d}$ saturates or even decrease with increasing wind speed (Donelan et al. 28, Fan et al. 39, Hara and Belcher 49, 50, Moon et al. 74, Reichl et al. 83). Constraining the parameterizations of $C_{d}$, wave growth, and dissipation over a range of wave spectral conditions, including waves misaligned with and opposing wind, remains the focus of a very active area of research.

Several studies have aimed to address the effects of wind-wave misalignment (difference of the wave direction from the wind speed direction, $\theta$ ) on wave growth and dissipation (e.g. Ardhuin et al. 3, Kudryavtsev and Makin 59, Meirink et al. 69, Tolman and Chalikov 98), as well as on the wind stress vector and $C_{d}$ (Bourassa 
et al. 10, Geernaert 41, Geernaert et al. 42, Grachev et al. 46, Suzuki et al. 95). Misalignment between wind and dominant surface waves occurs frequently in the open ocean over swells with low-to-moderate wind conditions (Ardhuin et al. 3, Donelan and Dobson 29, Donelan et al. 31, Drennan et al. 32, Edson et al. 34, Grachev et al. 46, Högström et al. 54, Patton et al. 78) as well as transient high wind conditions coupled to complex seas (Black et al. 9, Fan et al. 39, Holthuijsen et al. 55, Walsh et al. 104, Wright et al. 106). Due to the challenges of in-situ observations, the effects of wind-wave misalignment on the wind stress vector, drag coefficient, wave growth and dissipation remain poorly constrained. Even with typical wave fields with dominant waves aligned with wind, the real ocean wave spectra always contain significant contributions from misaligned waves. Therefore, improved understanding of interaction between wind and misaligned waves is essential for the modeling efforts of sea-state dependent drag coefficient in order to account for their impacts.

In Part I of this study, we have used LES to investigate the turbulent airflow over a steep wave train $(a k=0.27, k$ is wavenumber and $a$ is wave amplitude) following and opposing wind for $\left|c / u_{* s}\right|=1.4-11$ ( $c$ is wave phase speed and $u_{* s}$ is surface wind friction velocity). In particular, we have found a rather smooth transition in the near-surface airflow, from slow waves following wind to slow waves opposing wind to fast waves opposing wind, of increasingly stronger flow perturbations mainly caused by intermittent flow separations or separation-like patterns. The wave decay rate rapidly increases as the opposing wave speed increases, and our results are consistent with the recent LES study by Cao et al. [16] and earlier observational and theoretical studies. We have also found that the effective roughness length (drag coefficient) rapidly increases as the opposing wave speed increases. 
In Part II of this study, we use an identical LES approach of turbulent airflow over surface waves to expand on Part I, by considering waves following and opposing wind at an oblique angle $\left(\theta=45^{\circ}\right)$. Previous observational studies have proposed empirical scaling coefficients of the wave growth rate such as $\cos (\theta)$ (e.g., Plant 81, Snyder et al. 88) and $\left(U_{\lambda / 2} \cos (\theta)-c\right)^{2}$ (e.g., Donelan 25, Donelan et al. 27, 28). Previous theoretical studies tend to point towards $\cos ^{2}(\theta)$ (e.g., Burgers and Makin 15, Li et al. 65, Mastenbroek 68, Meirink et al. 69).The latter two parameterizations are frequently used in existing wave prediction models. To our knowledge, the wave decay rate of waves opposing oblique wind has not been investigated. Furthermore, our understanding of the effects of obliquely propagating waves on the mean wind profile and drag coefficient remains limited.

The goal of this study (Part II) is to address how the wave growth/decay rate, mean wind profile (magnitude and direction), effective roughness length, and drag coefficient are modified by steep, strongly forced waves following/opposing oblique wind $\left(|c| / u_{* s}=1.4, a k=0.27\right)$.

\subsection{Methods}

\section{LES setup}

We use an LES methodology of turbulent airflow over surface waves that is identical to previous studies (Hara and Sullivan 51, Husain et al. 56, Sullivan et al. 90, 93 and Part I), all of which use pressure-driven channel flow over a wavy surface propagating through a domain with doubly periodic horizontal boundary conditions and a free slip flat top boundary. Here, we define time $t$, the along-wave $x$-coordinate, the cross-wave $y$-coordinate, and the vertical coordinate $z$ pointing 
upward in the positive direction with $z=0$ at the mean water surface. Velocities $(u, v, w)$ are in the $(x, y, z)$ directions.

We consider a monochromatic wave train propagating in the $x$-direction $\left(\frac{\partial}{\partial y}=0\right)$ with $h(x, t)=a \cos (k x-\omega t)$, where $a$ is the wave amplitude, $k$ is the wavenumber, $\omega$ is the angular frequency, and $c=\omega / k=\sqrt{g / k}$, identical to Part I. In LES, wind is driven by imposed horizontal pressure gradient $\nabla p=\left(\frac{\partial p}{\partial x}, \frac{\partial p}{\partial y}\right)$, which is balanced by the surface wind stress $\left|\tau_{s}\right|=u_{* s}^{2}=|\nabla p| l_{\zeta}$, where $l_{\zeta}$ is the domain height. The direction $\theta$ of total wind stress vector is always directed in the direction of $-\nabla p$, the angle of external negative pressure gradient. Since we apply the free slip condition at the top boundary, the total wind stress linearly decreases from the wavy surface to the top.

For the actual LES, the waves always propagate in the positive $x$ direction and the pressure gradient force (i.e., negative pressure gradient) is applied in the directions of $0,45,180$, and 225 degrees (measured from the $x$ axis in the counterclockwise direction). However, for the data analysis and discussion, the wave direction for the last two cases is reversed. Therefore, for the four wind-wave conditions examined in this study, the wave directions are $\left(0^{\circ}, 0^{\circ}, 180^{\circ}, 180^{\circ}\right)$ and the wind stress directions $\theta$ are $\left(0^{\circ}, 45^{\circ}, 0^{\circ}, 45^{\circ}\right)$, respectively.

The subgrid and surface stress parameterizations are identical to those in Part I. The background surface roughness $z_{o b}$ along the resolved wavy surface is set at $k z_{o b}=2.7 \times 10^{-3}$, which accounts for the form drag of unresolved waves and the surface viscous stress. As in Part I we focus on a steep wave train of $a k=0.27$. The wind forcing is set at $|c| / u_{*}=1.4$ because we are mainly interested in strongly forced waves that support a bulk of the air-sea momentum flux (Donelan et al. 28, Reichl et al. 83). 
Each simulation is run for approximately 100,000 time steps and averaged over the last 20,000 time steps after the wind field has reached a statistically steady state. Sullivan et al. [93] and Sullivan et al. [90] provide a full description of the LES algorithm and numerical methods used to solve the governing equations.

\section{Data analysis}

As in Part I we use a wave-following mapping and a coordinate system moving with the wave such that the wave shape is frozen and $h(x, t)$ becomes $h(\xi)=$ $a \cos (k \xi)$. For more information on the wave-following mapping and the Jacobian transformation, refer to Hara and Sullivan [51] and Part I. In particular, the contravariant vertical velocity perpendicular to the wave shape and constant $\zeta$ surfaces is defined as:

$$
W=\frac{1}{J} u \frac{\partial \zeta}{\partial x}+w
$$

where $J$ is Jacobian, and is used to define $u W$ and $v W$ as vertical fluxes of $x-$ and $y$-momentum across constant $\zeta$ surfaces due to the advective velocity $W$.

Using the triple decomposition (separating all relevant variable $\psi$ into phase average $\bar{\psi}$ and turbulent $\psi^{\prime}$ component, and separating $\bar{\psi}$ into horizontal mean $\langle\psi\rangle$ and wave coherent component $\tilde{\psi}$, see Buckley and Veron 12, Hara and Sullivan 51, Husain et al. 56, and Part I), we can analyze the partition of the wind stress (momentum budget) in terms of turbulent and wave-coherent stresses as well as pressure stress in the along-wave direction. The addition of oblique wind adds a cross-wave $(y)$ momentum budget to the along-wave $(x)$ budget.

Specifically, the $x$ and $y$ normalized momentum equations may be expressed as

$$
\frac{\left\langle\tau_{13}^{w}\right\rangle+\left\langle\tau_{13}^{p}\right\rangle}{\tau_{s}}+\frac{\left\langle\tau_{13}^{t}\right\rangle}{\tau_{s}}+\frac{\frac{\partial P}{\partial x} \zeta}{\tau_{s}}=\cos (\theta)
$$




$$
\frac{\left\langle\tau_{23}^{w}\right\rangle}{\tau_{s}}+\frac{\left\langle\tau_{23}^{t}\right\rangle}{\tau_{s}}+\frac{\frac{\partial P}{\partial y} \zeta}{\tau_{s}}=\sin (\theta)
$$

where $\theta$ is the direction of the total wind stress (applied negative pressure gradient),

$\left\langle\tau_{13}^{w}\right\rangle=\langle\tilde{u} \tilde{W}\rangle$ and $\left\langle\tau_{23}^{w}\right\rangle=\langle\tilde{v} \tilde{W}\rangle$ are the along-wave and cross-wave components of the wave-coherent stress, and $\left\langle\tau_{13}^{t}\right\rangle=\left\langle u^{\prime} W^{\prime}\right\rangle$ and $\left\langle\tau_{23}^{t}\right\rangle=\left\langle v^{\prime} W^{\prime}\right\rangle$ are the along-wave and cross-wave components of the turbulent stress (including both the resolved and parameterized subgrid-scale stress). The pressure (form) stress is defined as $\tau_{13}^{p}=\frac{1}{J} \bar{p} \frac{\partial \zeta}{\partial x}$ (see Hara and Sullivan 51 and Part I) and is only present in the $x$ momentum equation because the wave shape does not change in the cross-wave direction.

\subsection{Results and discussion}

\section{D phase-averaged airflow}

In this section all flow fields presented are normalized by $u_{* s}$ and $k$. In Figures 3.1 (except the rightmost column), 3.2, 3.3, and 3.4 we display the two-dimensional phase-averaged flow fields. In the top (bottom) two rows waves propagate from left to right (from right to left) in the positive (negative) $x$ direction. In the first and third rows wind blows from left to right in the positive $x$ direction. In the second and fourth rows the pressure gradient force and the resulting wind stress are in the direction rotated by $45^{\circ}$ from the positive $x$ direction. Consequently, the wind direction is also positively rotated from the $x$ direction (by more than $45^{\circ}$, as discussed later). All the phase averaged flow fields are presented in the coordinate moving with the wave so that the flow fields are independent of $t$.

Figure 3.1 includes the streamwise velocity $\left((\bar{u}-c) / u_{* s}\right)$, spanwise velocity 
$\left((\bar{v}) / u_{* s}\right)$, vertical velocity $\left(\bar{w} / u_{* s}\right)$, and pressure $\left(\bar{p} / u_{* s}^{2}\right)$ plotted in rectangular $(\xi-z)$ coordinates. In the rightmost column, the surface stress distribution is plotted for the total normal stress (pressure plus the turbulent normal stress), pressure only, and the along-wave turbulent tangential stress. Figure 3.2 includes the TKE magnitude $\left(\bar{e} / u_{* s}^{2}\right)$, the dissipation rate $\left(\epsilon /\left(k u_{* s}^{3}\right)\right)$, and the horizontal vorticity magnitude $\left(\overline{\omega_{h}} /\left(k u_{* s}\right)=\sqrt{{\overline{\omega_{x}}}^{2}+{\overline{\omega_{y}}}^{2}} /\left(k u_{* s}\right)\right.$. Here, $\overline{\omega_{x}}=-\frac{\partial \bar{v}}{\partial z}+\frac{\partial \bar{w}}{\partial y}$ and $\overline{\omega_{y}}=\frac{\partial \bar{u}}{\partial z}-\frac{\partial \bar{w}}{\partial x}$ are dominated by the vertical shear of $\bar{v}$ and $\bar{u}$, respectively. Therefore, $\overline{\omega_{h}}$ is dominated by the vertical shear of oblique horizontal wind. Figure 3.3 shows the horizontal vorticity magnitude (same as Figure 3.2) and the two components of the horizontal vorticity, $\overline{\omega_{y}} /\left(k u_{* s}\right)$ and $-\overline{\omega_{x}} /\left(k u_{* s}\right)$. Figures 3.4 includes the three components of the TKE. All quantities in Figures 3.2, 3.3, and 3.4 are plotted in the rectangular $(\xi-z)$ coordinate as well as in the mapped $(\xi-\zeta)$ coordinate with the vertical axis in a log scale so that the flow fields very close to the wavy surface are magnified. Note that the results for waves following and opposing wind (first and third rows) are almost identical to the results presented in Part I for $c / u_{* s}= \pm 1.4$

First, we examine the phase averaged velocity and pressure fields in Figure 3.1. In the oblique wind cases with waves following or opposing wind, the along-wave velocity (in the $x$ direction) is reduced, but the overall patterns remain very similar to those of the aligned wind cases (Figure 3.1a-d). The cross-wave velocity (in the $y$ direction) is introduced in the oblique cases (Figure 3.1e,f) and is much stronger than the along-wave velocity (Figure 3.1b,d). We will later show that even in a fixed coordinate, $\langle\bar{v}\rangle$ is larger than $\langle\bar{u}\rangle$, that is, the mean wind direction is rotated from the $x$ direction by more than $45^{\circ}$. This is expected, at least qualitatively, because waves exert more friction (due to the wave form drag) and wind speed is 
more reduced in the along-wind $(x)$ direction.

With the oblique wind, both the along-wave and cross-wave velocities are significantly reduced over the leeward face of the wave (Figure 3.1b,d,e,f), suggesting that intermittent airflow separations (or separation-like flows) are modifying the wind fields, as in the aligned wind cases (Figure 3.1a,c). The vertical velocity is slightly weaker in the oblique cases, but the patterns remain largely unchanged from the aligned cases (Figure 3.1g-j).

As expected, the pressure along the wave phase weakens with oblique wind as less wind forcing is exerted onto the wave shape in the $x$-direction (Figure $3.1 \mathrm{k}-\mathrm{n}$ ), resulting in weaker surface pressure and total normal stress than in the aligned cases (Figure 3.1A,C). Interestingly, the surface tangential stress in the along-wave direction is even more reduced with oblique winds (Figure 3.1B,D), possibly because the horizontally averaged wind vector very close to the surface is rotated by almost $65^{\circ}-68^{\circ}$ from the $x$ direction, as discussed later.

Next, we examine the phase averaged fields of TKE, dissipation rate, and horizontal vorticity magnitude in Figure 3.2. For both aligned and oblique cases, regions of enhanced dissipation and vorticity appear to detach from the crest and extend downstream above the dead zone of significantly reduced TKE, dissipation rate, and vorticity on the leeward face of the wave. The enhanced TKE above the crest appears to be advected by the high velocity just above the detached high vorticity layer. These patterns are quite similar between the aligned and oblique wind cases, and suggest that flow is intermittently separating from the crest (or exhibiting separation-like patterns) even in oblique winds.

In general, the opposing and following wave cases in oblique winds are similar, except that the faster relative wind speed in the opposing case tends to limit the 
vertical extent of the wave induced flow perturbations closer towards the surface (compare Figure 3.2b,f,j and Figure 3.2d,h,l). The same trend has been observed in the aligned wind cases in Part I and also in Figure 3.2.

One notable difference exists between the oblique and aligned wind cases. The high vorticity and high dissipation regions along the wavy surface are mostly confined near the crest in the aligned wind cases (Figure 3.2E,G,I,K). However, these regions extend upstream all the way to the wave trough in the oblique wind cases (Figure 3.2F,H,J,L). Consequently, the reduction of dissipation rate and vorticity in the dead zone is muted with the oblique wind (Figure 3.2F,H,J,L).

To shed more light on these features, we next examine the along-wave and cross-wave vorticity components separately in Figure 3.3. It is clear that the detached layer of enhanced vorticity is present in both directions (Figure 3.3F,I,H,J) in the oblique cases. The cross-wind vorticity simply weakens in oblique winds, but its patterns remain very similar between the aligned and oblique cases with reduced vorticity in the dead zone (Figure 3.3E-H). In contrast, the along-wave vorticity remains strong along the entire surface, and especially along the windward face and the crest of the wave (Figure 3.3I,J). This enhanced along-wind vorticity appears to be well correlated with the enhanced dissipation in the same location (Figure $3.2 \mathrm{~F}, \mathrm{H})$. The enhancement of along-wind vorticity and dissipation rate on the windward side of the crest (between the trough and following crest) may be related to reattachment of the separated oblique wind flow. We will later show that the cross-wave wave-coherent velocity $\tilde{v}$ is also enhanced there.

One interesting feature for waves opposing oblique winds is the presence of a region of negative cross-wave vorticity near the trough (Figure 3.3H). In Part I, we have shown that for waves opposing wind a region of negative cross-wave vorticity 
near the trough intensifies as waves get faster, and is associated with separation-like detachment of airflow from the surface. In such a region wind shear is negative; that is, the wind speed decreases with height. However, in the case of oblique wind, the mean wind direction is close to $70^{\circ}$ (as explained later) and the along-wave vorticity remains strong in the same region. Therefore, the wind speed magnitude increases with height.

In Figure 3.4, we separate the TKE into along-wave $\left(0.5 \overline{u^{\prime} u^{\prime}} / u_{* s}^{2}\right)$, cross-wave $\left(0.5 \overline{v^{\prime} v^{\prime}} / u_{* s}^{2}\right)$, and vertical $\left(0.5 \overline{w^{\prime} w^{\prime}} / u_{* s}^{2}\right)$ components. As expected, in aligned winds the TKE is dominated by the along-wave component (Figure 3.4a,c,A,C). One notable exception is a region windward side of the crest near the surface, where the cross-wave component is significantly enhanced and is larger than the along-wave component (Figure 3.4e,g,E,G). The cause of this enhancement is not clear, but it may be related to the reattachment of separated flow in this area.

In the oblique case, the along-wave component of the TKE is drastically reduced and the cross-wave component is significantly larger. This is consistent with the fact that both mean wind speed and mean wind shear are rotated by more than $45^{\circ}$ from the $x$-axis, as discussed later. The enhancement of the cross-wave component on the windward side of the crest is observed in the oblique wind cases as well (Figure 3.4f,h,F,H). This enhancement is possibly related to the enhanced along-wind vorticity (Figure 3.3I,J) with large $\frac{\partial \bar{v}}{\partial z}$ in the same area. Finally, the consistently small vertical component suggests that the airflow turbulence is dominated by horizontal velocity variances in the entire wave boundary layer. 


\section{Horizontally averaged wind profiles in mapped coordinates}

Next, we investigate the vertical profiles of the horizontally averaged wind variables in the mapped $(\zeta)$ coordinate, including mean wind speed, mean wind shear, TKE and its components, shown in Figure 3.5. In the following subsections profiles for waves following (opposing) aligned wind are shown as thick orange (blue) lines, and profiles for waves following (opposing) oblique wind are shown as thin orange (blue) lines. All the profiles are displayed up to $k \zeta=2$ because the results above this elevation are affected by the reduced wind stress and the LES top boundary.

The four panels in the top row of Figures 3.5 display vertical profiles of the horizontally averaged mean wind speed vector. The $x$ and $y$ components of the wind vector, $\langle u\rangle / u_{* s}$ and $\langle v\rangle / u_{* s}$, are shown in Figures 3.5a and 3.5d, respectively, while

their magnitude, $\left(\langle u\rangle^{2}+\langle v\rangle^{2}\right)^{1 / 2} / u_{* s}$, and angle, $\theta=\arctan (\langle v\rangle /\langle u\rangle)$, are shown in Figures 3.5g and 3.5j, respectively. The grey lines in Figures 3.5a,d represent the flat wall wind speed profiles with a background roughness of $k z_{o b}=2.70 \times 10^{-3}$, for aligned (thick lines) and oblique (thin lines) cases, for linearly decreasing wind stress (solid) and constant wind stress (dashed) in $k \zeta$. In Figures 3.5g the thick grey lines apply for both aligned and oblique cases. The thin dashed grey line in Figure 3.5j represents the angle of the flat wall wind speed profile, $\theta=45^{\circ}$, which is equal to the angle of the wind stress and the imposed pressure gradient.

As discussed in Part I, the wind speed magnitude (Figure 3.5g) in the aligned cases (thick orange and blue lines) is significantly reduced from the wind profile for a flat wall (thick grey line) near the top of the domain, indicating that the equivalent surface roughness is increased for both waves following and opposing 
wind, with the latter having a slightly higher roughness length. However, the wind speed magnitude in the oblique cases (thin orange and blue lines) is very close to that for a flat wall (thick grey line) near the top of the domain for both waves following and opposing the oblique wind, which indicates that the waves do not enhance the equivalent surface roughness above the background roughness. As in Part I, we estimate the enhancement of the equivalent surface roughness $z_{o}$ relative to the background roughness $z_{o b}$ by comparing the wind speed magnitude at the top of the domain $(k \zeta=2)$ with and without waves. We find that $z_{o} / z_{o b}=4.60$ and 6.36 for waves following and opposing aligned wind, and $z_{o} / z_{o b}=0.90$ and 1.10 (practically no enhancement) for waves following and opposing oblique wind (Table 3.1).

If we examine the along-wave and cross-wave wind speeds separately (Figure $3.5 \mathrm{a}, \mathrm{d}$ ) for the oblique cases, it is clear that both components are significantly modified by the wave. As expected the along-wave wind speed is reduced from the flat wall profile because of the wave form drag. The along-wave wind profiles for oblique wind are roughly proportional to those for aligned wind, and are reduced by about 25-30\% throughout the wave boundary layer. Interestingly, the cross-wave wind speed is increased near the top of the domain compared to the flat wall wind speed (Figure 3.5b), and this increase compensates the decrease of the along-wave wind speed, yielding the almost unchanged wind speed magnitude toward the top.

The opposite wave impacts on the along-wave and cross-wave wind speeds mean that the angle of the wind speed $\left(\theta=52^{\circ}-54^{\circ}\right)$ is significantly misaligned from the angle of the wind stress $\left(\theta=45^{\circ}\right)$ near the top of the domain (Figure 3.5j), even though the wind magnitude is not modified by the wave. The wind speed angle is much larger closer to the surface, falling between $\theta=65^{\circ}$ and $\theta=68^{\circ}$. From 
about $k \zeta=0.1$ to 0.4 , the angle quickly reduces to about $\theta \approx 55^{\circ}$, and reduces more slowly from there to the top at $k \zeta=2$. It is notable that the wind speed angle is strongly dependent on height in the wave boundary layer, and that its misalignment from the total wind stress angle $\left(\theta=45^{\circ}\right)$ appears to persist above the top of the wave boundary layer.

The second row of Figure 3.5 displays vertical profiles of the normalized mean wind shear. Figures 3.5b and 3.5e show the along-wave and cross-wave shear, $\left(\frac{\partial\langle u\rangle}{\partial \zeta} \frac{\kappa \zeta}{u_{* s}}\right)$ and $\left(\frac{\partial\langle v\rangle}{\partial \zeta} \frac{\kappa \zeta}{u_{* s}}\right)$, respectively, and Figures 3.5h and 3.5k show the wind shear magnitude and the wind shear angle (solid lines), respectively. The grey lines represent the flat wall wind shear profiles as in the first row.

The shape of vertical profiles of wind shear magnitude (Figures 3.5h) is quite similar between the aligned and oblique wind cases, with a reduction near the surface and enhancement at mid-level. However, the wind shear magnitude for the oblique wind is consistently larger throughout the wave boundary layer (compare thin orange line with thick orange line, or thin blue line with thick blue line). This larger wind shear magnitude is responsible for the larger wind speed near the top of the domain (Figures 3.5g) and the resulting smaller equivalent roughness length.

The along-wave wind shear profiles (Figure 3.5b) for the oblique wind cases are roughly proportional to those for the aligned wind cases (except near the top where they collapse); they are reduced by about 25-30\%. This suggests that the same physical processes (i.e. effects of pressure form drag near the surface, effects of intermittent airflow separations at mid-level) take place in oblique wind cases, but their impacts are reduced. Near the top of the wave boundary layer, the along-wave wind shear is not much reduced for the oblique cases, and this contributes to the enhanced wind shear magnitude near the top. 
In the middle of the wave boundary layer, the cross-wave wind shear profiles (Figures 3.5e) for the oblique wind cases are significantly enhanced compared to that for a flat wall case, and this contributes to the enhancement of the wind shear magnitude and far field wind speed as well as the reduced equivalent roughness length. The shear enhancement occurs at lower elevation with waves opposing (compared to following) oblique wind, which is consistent with the earlier observation that waves opposing wind tend to suppress the vertical extent of the wave induced flow perturbations.

The angle of the mean wind shear is plotted in Figure 3.5k (solid lines), along with the angle of the horizontally averaged turbulent stress (dashed lines; more discussion on the turbulent stress and momentum budget in the next subsection). Both the wind shear angle and the turbulent stress angle hover around $\theta=45^{\circ}$ above $k \zeta=1$; that is, the mean wind shear and the turbulent stress are well aligned with the total wind stress there. This suggests that the direction of the mean wind speed gradually approaches the wind stress direction (i.e., the misalignment between wind speed and wind stress gradually disappears) if the constant stress layer is extended upward without a top boundary (i.e., in the open ocean condition).

At mid-level, the wind shear angle oscillates around $45^{\circ}$ for waves opposing wind, but it is significantly reduced from $45^{\circ}$ at around $k \zeta=0.30$ for waves following wind, which is associated with the enhancement of the along-wave wind shear (Figure 3.5b). Towards the surface, the wind shear angle increases to about $\theta=63^{\circ}$.

The profiles of the wind shear angle (solid lines) are generally well correlated with the profiles of the turbulent stress angle (dashed lines). This observation supports the common turbulence closure assumption that the angle of the turbulent wind stress is the same as the angle of the mean wind shear (that is, the momentum 
flux is downgradient). However, toward the surface the angle of the turbulent stress is consistently smaller than the angle of the mean wind shear by about $8^{\circ}-9^{\circ}$.

The third (bottom) row of Figure 3.5 displays vertical profiles of the horizontally averaged TKE and its three components. With the oblique wind, the magnitude of TKE remains largely unchanged (Figure 3.5c). However, the profiles of along-wave TKE and cross-wave TKE significantly differ between the oblique and aligned cases. In aligned winds, the along-wave TKE component is much larger and is pronounced at mid-level, associated with flow separation (or separation-like) patterns (see also Figure f3A,C). In oblique winds, the cross-wave TKE component is larger, and both components show modest enhancement at mid-level (see also Figure f3B,D,F,H). The vertical TKE component remains significantly smaller throughout the wave boundary layer.

\section{Horizontally averaged momentum budget in along-wave and cross-wave directions}

The horizontally averaged momentum budget (or wind stress partition) for the along-wave and cross-wave directions, as described in Equation 3.2, is shown in Figure 3.6. Similar to Figure 3.5, the solid (dashed) grey lines represent the values for the flat wall case with linearly decreasing (constant) total wind stress in $k \zeta$. The total stress $\left(\left\langle\tau_{13}^{\text {tot }}\right\rangle,\left\langle\tau_{23}^{\text {tot }}\right\rangle\right)$, which has been calculated by adding the wave-coherent stress $\left(\left\langle\tau_{13}^{w}\right\rangle,\left\langle\tau_{23}^{w}\right\rangle\right)$, turbulent stress $\left(\left\langle\tau_{13}^{t}\right\rangle,\left\langle\tau_{23}^{t}\right\rangle\right)$, and pressure stress $\left(\left\langle\tau_{13}^{p}\right\rangle, 0\right)$, is almost identical to the grey solid lines, indicating that the momentum budget is well satisfied in both directions.

In Figure 3.6a,c the vertical profiles of all stress components in the along-wave direction are similar between the aligned and oblique cases; the values in the oblique 
case are simply reduced roughly in proportion to the reduction of total stress. In both cases, the pressure stress magnitude increases toward the surface, and the wave-coherent stress is positively (upward momentum flux) enhanced at mid-level $(k \zeta \approx 0.3-0.5)$. The turbulent stress magnitude is reduced near the surface to compensate the pressure stress, and is enhanced at mid-level to compensate the wave-coherent stress.

In Figures 3.6b,d, the pressure stress is zero in the cross-wave direction with no wave shape, but the turbulent and wave-coherent stresses are strongly modulated by the wave. In particular, for waves following oblique wind the negative wavecoherent stress $\left\langle\tau_{23}^{w}\right\rangle$ (downward momentum flux) is significantly enhanced around $k \zeta=0.2-0.3$, and the turbulent stress $\left\langle\tau_{23}^{t}\right\rangle$ is reduced to compensate it. This reduction of $\left\langle\tau_{23}^{t}\right\rangle$ is reflected in the decrease of the turbulent stress direction at the same level (Figure 3.5k), which is well correlated with the reduced mean wind shear direction (Figure 3.5k). Therefore, the wave-coherent stress seems to play an important role in modifying the mean wind profile.

To better understand the wave modulated turbulent and wave-coherent stresses, we examine the phase-averaged 2D fields of turbulent stress, wave-coherent stress, and wave-coherent velocities, $\tilde{u}, \tilde{v}, \tilde{W}$, in Figure 3.7. In the along-wave direction, the $2 \mathrm{D}$ pattern of wave-coherent stress $\overline{\tau_{13}^{w}}=\tilde{u} \tilde{W}$ is very similar in all cases (Figures 3.7g-j). Strong upward momentum flux (positive $\tilde{u} \tilde{W}$ ) occurs in two areas due to flow separation and reattachment. Namely, just downstream the crest the accelerated fluid (positive $\tilde{u}$ ) is carried away from the surface (positive $\tilde{W}$ ), and just downstream of the trough the decelerated fluid (negative $\tilde{u}$ ) returns toward the surface (negative $\tilde{W}$ ) (Figures $3.7 \mathrm{~m}-\mathrm{t}$ ). The combined effect introduces large upward momentum flux if averaged horizontally (Figures 3.6c). In oblique wind, the 
along-wave wave-coherent velocities and stresses are weakened, but their patterns remain qualitatively similar. To compensate the upward wave-coherent stress, the negative turbulent stress $\overline{\tau_{13}^{t}}$ is significantly enhanced above the windward side of the crest (dark blue regions in Figures 3.7a-d).

A pair of similar but much weaker areas of positive wave-coherent stress $\left(\overline{\tau_{23}^{w}}=\right.$ $\tilde{v} \tilde{W}$ ) are observed in the cross-wave direction as well (red areas in Figures 3.6k,l). However, they are dwarfed by a pair of much stronger downward momentum flux (negative $\tilde{v} \tilde{W}$ ) regions (blue areas in Figures $3.6 \mathrm{k}, 1$ ). This downward flux is caused by the large perturbation of $\tilde{v}$ along the wave phase. Namely, $\tilde{v}$ is negative between the leeward crest and the leeward trough, and is positive between the windward trough and the following crest. The separating flow (positive $\tilde{W}$ ) carries the fluid with negative $\tilde{v}$ away from the surface, and reattaching flow (negative $\tilde{W})$ brings the fluid with positive $\tilde{v}$ back toward the surface (Figures $3.6 \mathrm{~m}-\mathrm{p}, \mathrm{u}, \mathrm{v}$ ). The resulting downward flux is particularly strong for waves following oblique wind. This enhanced downward wave-coherent stress is compensated by the reduced downward turbulent stress (Figures 3.6e,f).

\section{Energy budget and turbulence closure parameterization}

Next, we plot the energy budget inside the wave boundary layer (Figure 3.7). As discussed in Part I, we use the derivation from Hara and Sullivan [51] for the equations governing the wave-fluctuation energy, $E^{w}=(1 / 2)(\tilde{u} \tilde{u}+\tilde{v} \tilde{v}+\tilde{w} \tilde{w})$, and the turbulent kinetic energy, $\bar{e}=(1 / 2)\left(\overline{u^{\prime} u^{\prime}}+\overline{v^{\prime} v^{\prime}}+\overline{w^{\prime} w^{\prime}}\right)$ in mapped coordinates. 
If the two equations are combined and normalized, the result yields:

$$
\begin{array}{r}
\left(\left\langle\tilde{u} \frac{1}{J}\right\rangle \frac{\partial P}{\partial x}+\left\langle\tilde{v} \frac{1}{J}\right\rangle \frac{\partial P}{\partial y}\right) \frac{\kappa \zeta}{u_{* s}^{3}}+\left(\left\langle\tau_{13}^{t o t}\right\rangle \frac{\partial\langle u\rangle}{\partial \zeta}+\left\langle\tau_{23}^{t o t}\right\rangle \frac{\partial\langle v\rangle}{\partial \zeta}\right) \frac{\kappa \zeta}{u_{* s}^{3}}-\frac{\partial\left(F^{w}+F^{t}\right)}{\partial \zeta} \frac{\kappa \zeta}{u_{* s}^{3}} \\
-\left\langle\frac{1}{J} \epsilon\right\rangle \frac{\kappa \zeta}{u_{* s}^{3}}=0
\end{array}
$$

where the second term is the shear production term (dark red lines), the third term is the transport term (light green lines; $F^{w}$ and $F^{t}$ are the vertical transport of $E^{w}$ and $\bar{e}$, respectively), and the fourth term is the viscous dissipation term (blue lines). The first term arises because of the imposed pressure gradient (i.e., because the stress is not constant in vertical) and is plotted in dark green, which is negligible. The sum of four energy budget terms is plotted as thin black lines, and indicates that the energy budget is well satisfied. Thin grey lines represent the mean shear production (mean wind shear) over a flat wall for linearly decreasing stress (solid) and constant stress (dashed) with respect to $k \zeta$. The thick and thin dashed black lines are the magnitudes of the turbulent stress profiles (replotted from Figure 3.6) for the aligned and oblique cases, respectively. Here, $\left(F^{w}+F^{t}\right)$ at the surface is equal to the energy flux into the waves. Refer to Hara and Sullivan [51] for more details on the derivation of the energy budget in mapped coordinates.

In all cases the transport term is relatively small and the shear production is mostly balanced by the viscous dissipation at all heights. For waves both following and opposing wind, the shear production for waves in oblique wind (thin lines) is significantly enhanced compared to that for waves in aligned wind (thick lines) throughout the wave boundary layer. The viscous dissipation for oblique wind (thin blue lines) is also enhanced and balances the enhanced shear production. 
As discussed earlier, the enhanced wind shear magnitude throughout the wave boundary layer in the oblique wind cases is responsible for the increased far field wind speed and reduced equivalent roughness length (to the point of almost wiping out the wave effect) compared to those in the aligned wind cases. The energy budget analysis here suggests that the enhanced wind shear and the reduced equivalent roughness length with oblique winds are correlated with the enhanced viscous dissipation throughout the wave boundary layer.

As discussed in Part I, previous modeling studies have closed the turbulence in the wave boundary layer by parameterizing the TKE dissipation rate $(\langle\epsilon / J\rangle)$ using the turbulent stress $\left(\left\langle\tau^{t}\right\rangle\right)$ (e.g., Hara and Belcher 50, Makin and Kudryavtsev 66). In Figure 3.8, we compare the magnitude of turbulent stress (dashed black lines) with the magnitude of viscous dissipation (blue lines). Their correlation is very strong in the aligned wind cases, but it is weaker in the oblique wind cases. In particular, if we compare the oblique cases (thin lines) with the aligned cases (thick lines), the turbulent stress is enhanced due to oblique wind only near the surface (roughly $k \zeta<0.1$ ) and is slightly reduced further above, while the viscous dissipation is enhanced everywhere. Therefore, the turbulence closure based on $\left(\left\langle\tau^{t}\right\rangle\right)$ would predict enhanced viscous dissipation near the surface and reduced dissipation above, that is, significantly underestimate the viscous dissipation integrated over the wave boundary layer. Then, it would underestimate the integrated mean wind shear and overestimate the resulting equivalent roughness length. This poses a challenge to existing modeling efforts of sea-state dependent drag coefficient. 


\section{Wave growth/decay rate}

As in Part I, we calculate the energy transfer rate $\beta$ from wind to waves using the surface stresses shown in Figures 3.1A-D, and use the following common expression to compute the wave growth/decay rate coefficient $c_{\beta}$ (positive/negative for growth/decay) for all conditions:

$$
\beta=c_{\beta}\left(\frac{u_{* s}}{c}\right)^{2} \frac{\rho_{a}}{\rho_{w}} \omega
$$

where $\omega$ is the wave frequency. The coefficient $c_{\beta}$ is then evaluated based on the total energy flux $\left(c_{\beta t o t}\right)$, and including the contributions from the tangential turbulent stress only $\left(c_{\beta t}\right)$, the normal turbulent stress only $\left(c_{\beta n}\right)$, and the pressure only $\left(c_{\beta p}\right)$. These quantities are summarized in Table 3.1. Note that in some literature $c_{\beta}$ is defined for the aligned wind-wave condition and the impact of misalignment $\theta$ between waves and wind stress is explicitly added. Here, our $c_{\beta}$ includes the misalignment effect.

The reduction of the pressure component only $\left(c_{\beta p}\right)$ due to the misalignment of $\theta=45^{\circ}$ is consistently close to $50 \%$ for both following and opposing oblique cases (48\% and 53\%, respectively), which is quite consistent with the most common parameterization, $\cos ^{2}(\theta)$, of the misalignment effect. This also means that the pressure form drag is reduced by about 50\%. Interestingly, if all turbulent stress contributions are added, the reduction of the total wave growth/decay rate coefficient $c_{\beta t o t}$ due to wind-wave misalignment changes to about $58 \%$, which is above the $\cos ^{2}(\theta)$ dependence but still less than the $\left(U_{\lambda / 2} \cos \theta-c\right)^{2}$ dependence $(63-64 \%)$ (Donelan et al. 28, Reichl et al. 83).

In summary, the wave form drag and the wave growth rate are both reduced due 
to misalignment $\theta$ between waves and wind stress, and the common parameterization of the angle dependence, $\cos ^{2}(\theta)$, appears to be approximately valid. While the wave form drag remains significant (reduced by only about $1 / 2$ ), the wave impact on the equivalent roughness length almost disappears due to the misalignment. The latter finding is one of the most significant and surprising results of this study. This finding also suggests that existing models of sea-state dependent drag coefficient may overestimate the impact of misaligned waves if the drag coefficient is simply assumed to increase with increasing wave form drag integrated over the entire wave spectrum.

\subsection{Summary}

In this study, we use large eddy simulation to investigate turbulent airflow over steep strongly forced waves $\left(|c| / u_{* s}=1.4, a k=0.27\right)$ following and opposing oblique wind (with wind stress direction misaligned from wave direction by $\theta=45^{\circ}$ ) as well as following and opposing aligned wind. Our results show that the phase averaged airflow in oblique wind maintains a signature of intermittent airflow separations characterized by enhanced vorticity originating along the windward face and detaching from the crest of the wave, resulting in reduced wind speed, TKE, vorticity, and dissipation in the leeward trough. These features in oblique wind cases appear to be as strong as those in aligned wind cases (Figure 3.2).

These airflow features appear to modify the pressure fields and the resulting surface form drag due to oblique wind (Figure $3.1 \mathrm{k}-\mathrm{n}, \mathrm{A}-\mathrm{D}$ ), resulting in wave growth/decay rate ( $c_{\beta p}$, due to pressure stress alone) and the pressure form drag reduced by around 50\% from the aligned wind case. With the inclusion of the turbulent stress components the reduction of $c_{\beta t o t}$ is about $58 \%$ for both following 
and opposing waves (Table 3.1). These results indicate that the common parameterization for the wind-wave angle dependence of $\cos ^{2}(\theta)$ seems to be reasonably appropriate.

With waves following/opposing oblique wind, the mean wind speed (Figure $3.5 \mathrm{a}, \mathrm{d}, \mathrm{g}, \mathrm{j}$ ) and the mean wind shear (Figure 3.5b,e,h,k) are strongly modified. The directions of mean wind speed and mean wind shear significantly deviates from the direction of the wind stress $\theta=45^{\circ}$ (Figure 3.5j,k). Most notably, we see a significant turning of the wind angles towards the surface $\left(\theta=65^{\circ}-68^{\circ}\right.$ for wind speed, $\theta=63^{\circ}$ for wind shear). Toward the top of the wave boundary layer the wind shear angle becomes close to $\theta=45^{\circ}$, but the wind angle remains significantly misaligned $\left(\theta=52^{\circ}-54^{\circ}\right)$. Although the direction of the mean wind shear varies with height in a complex manner, it is well correlated with the direction of the turbulent stress throughout the wave boundary layer, that is, the turbulent momentum flux remains mostly downgradient (Figure 3.5k).

The momentum budget (partition of the total wind stress into turbulent, wavecoherent, and pressure stress components) in the along-wave direction (Figure 3.6a,c) is quite similar in oblique and aligned wind cases, with enhanced positive wavecoherent stress (upward momentum flux) at mid level due to airflow separations, pressure stress increasing toward the surface (downward momentum flux), and turbulent stress balancing the two. An analysis of the momentum budget in the cross-wave direction (Figure 3.6b,d) highlights strong mid-level downward wavecoherent stress balanced by the reduced cross-wave turbulent stress. This feature appears to be related to the separating and reattaching flows (Figure 3.7n,p,u,v), and responsible for significant turning of the mean wind shear direction (Figure 3.5k).

The most notable finding in this study is that waves oblique to wind appear to 
have little impact on the equivalent roughness length and the drag coefficient, even if such waves support significant wave form drag. We find that in oblique wind cases the wind shear magnitude is enhanced throughout the wave boundary layer (Figure 3.5h), the far field wind speed is significantly increased (Figure 3.5g), and the equivalent roughness length is reduced such that the impact of the waves is all but erased (i.e. the magnitude of the wind speed is not changed at the top of the wave boundary layer due to the oblique wave). The energy budget analysis shows that the enhanced shear production (i.e., enhanced mean wind shear) is balanced by enhanced viscous dissipation throughout the wave boundary layer (Figure 3.8) in the oblique wind cases. However, the turbulent stress in the wave boundary layer is not enhanced in a similar manner, which suggests that the existing turbulence closure schemes, relating the dissipation rate (or the mean wind shear) with the turbulent stress, may underestimate the mean wind shear and the far field wind speed and overestimate the equivalent roughness length and the drag coefficient in the presence of misaligned waves.

\subsection{Acknowledgements}

We acknowledge support of the National Science Foundation (Physical Oceanography) Grant OCE-1458984 (URI). We also acknowledge high-performance computing support from Yellowstone and Cheyenne (doi:10.5065/D6RX99HX) provided by NCAR's Computational and Information Systems Laboratory, sponsored by the National Science Foundation. 


\subsection{Figures and Tables}

Table 3.1: List of run conditions and results of roughness enhancement $z_{o} / z_{o b}$ and non-dimensional wave growth/decay coefficient $c_{\beta}$ for four LES simulations. The symbol $f$ and $o$ in the run name represents waves following and opposing wind, respectively; the subscript ' 45 ' represents oblique wind runs. The values for $c_{\beta t}, c_{\beta n}$, and $c_{\beta p}$ refer to tangential turbulent stress, normal turbulent stress, and pressure contributions to $c_{\beta}$, and $c_{\beta t o t}$ is the total.

$\begin{array}{lccccc}\text { Run } & z_{o} / z_{o b} & c_{\beta t} & c_{\beta n} & c_{\beta p} & c_{\beta t o t} \\ 1.4 f & 4.60 & 2.7 & 1.6 & 13.2 & 17.5 \\ 1.4 f_{45} & 0.90 & 0.6 & 3.2 & 6.4 & 10.2 \\ 1.40 & 6.36 & -3.5 & -0.5 & -13.0 & -17.0 \\ 1.4 o_{45} & 1.10 & -0.9 & -2.0 & -6.9 & -9.8\end{array}$



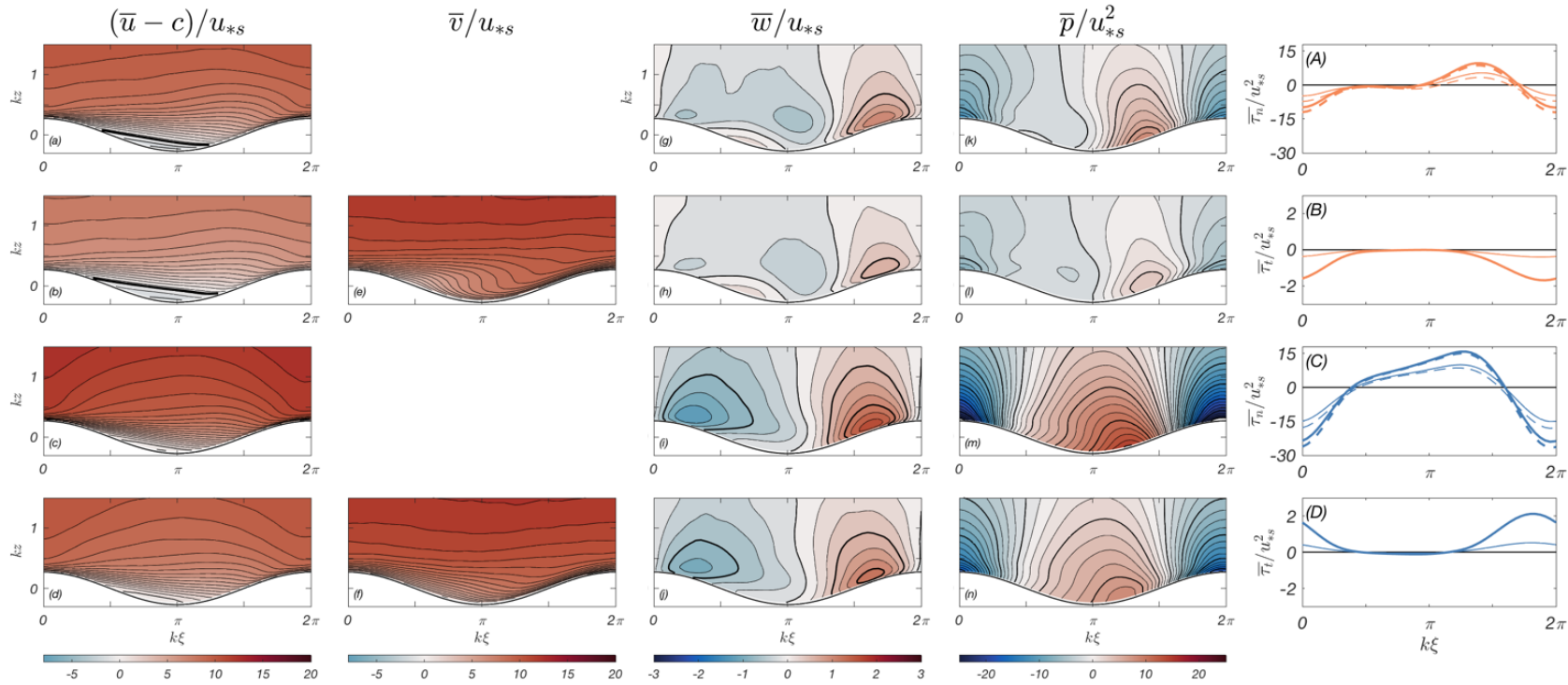

Figure 3.1: Normalized phase-averaged flow fields in the $\xi$-z coordinate for waves following wind (top two rows; oblique wind in second row) and for waves opposing wind (bottom two rows; oblique wind in fourth row). From left to right: along-wave velocity $\left((\bar{u}-c) / u_{* s}\right)$, cross-wave velocity $\left(v / u_{* s}\right)$, vertical velocity $\left(w / u_{* s}\right)$, and pressure $\left(\bar{p} / u_{* s}^{2}\right)$. Rightmost plots show the surface stress distribution for the normal stress $\overline{\tau_{n}} / u_{* s}^{2}$ (panels A and $\mathrm{C}$, solid line is total normal stress and dotted line is pressure only) and for the tangential stress $\overline{\tau_{t}} / u_{* s}^{2}$ in the $x$ direction (panels $\mathrm{B}$ and D) for waves following wind (orange lines, top two panels) and opposing wind (blue lines, bottom two panels) in aligned (thick lines) and oblique (thin lines) wind conditions. 

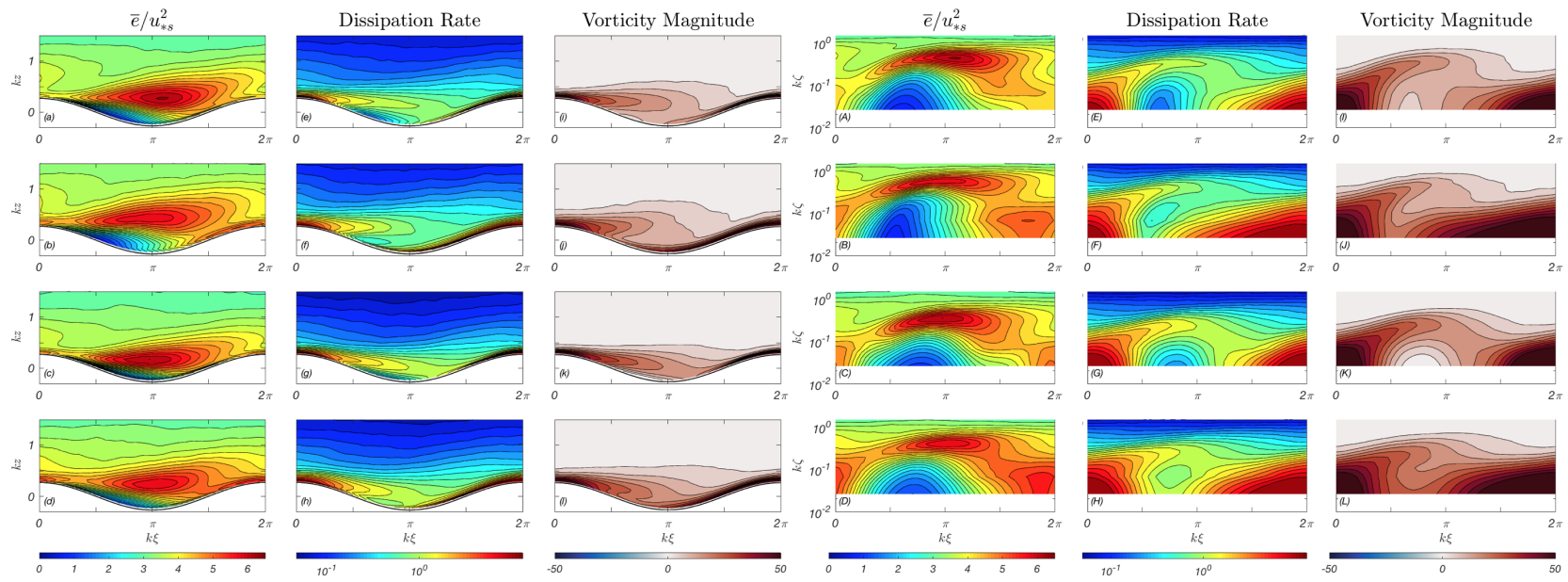

Figure 3.2: Normalized phase-averaged flow fields in the $\xi-z$ coordinate (left three panels) and the mapped $\xi-\zeta$ coordinate (right three panels) for waves following wind (top two rows; oblique wind in second row) and for waves opposing wind (bottom two rows; oblique wind in fourth row). From left to right: turbulent kinetic energy $\left(\bar{e} / u_{* s}^{2}\right)$, dissipation rate $\left(\epsilon /\left(k u_{* s}^{3}\right)\right)$, and vorticity magnitude $\left(\bar{\omega}_{h} /\left(k u_{* s}\right)\right)$.
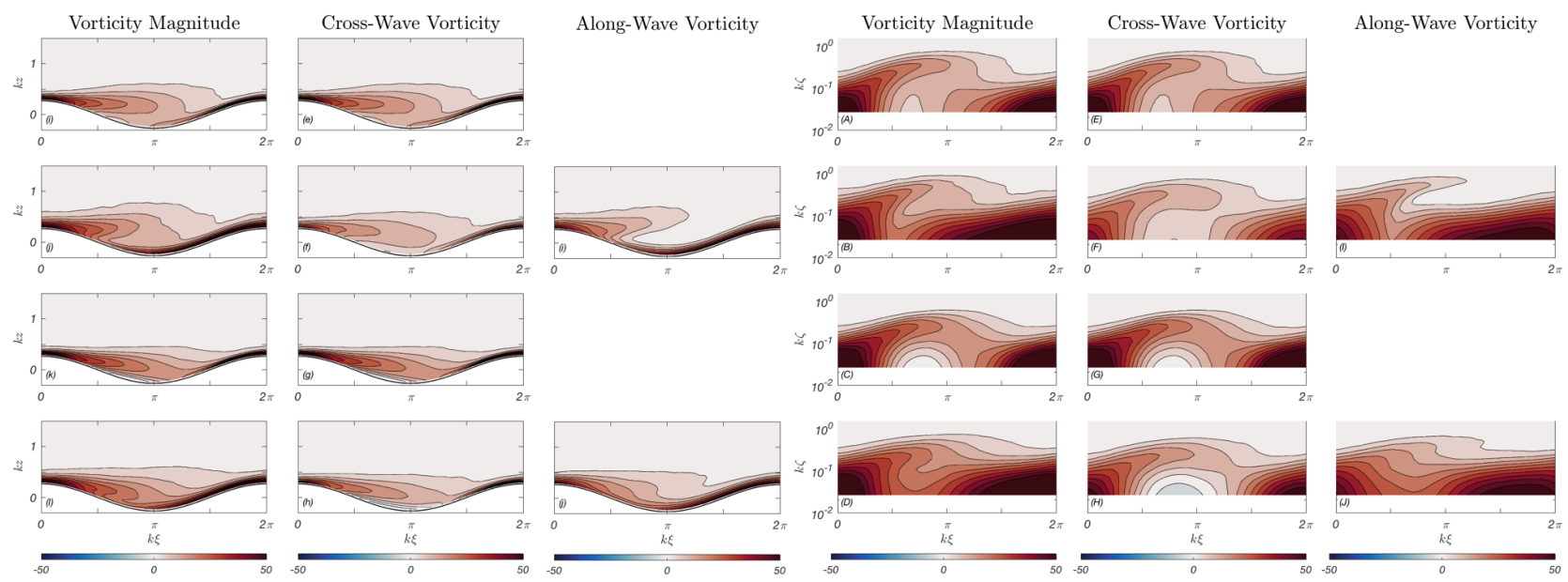

Figure 3.3: Normalized phase-averaged flow fields in the $\xi-z$ coordinate (left three panels) and the mapped $\xi-\zeta$ coordinate (right three panels) for waves following wind (top two rows; oblique wind in second row) and for waves opposing wind (bottom two rows; oblique wind in fourth row). From left to right: vorticity magnitude $\left(\bar{\omega}_{h} /\left(k u_{* s}\right)\right)$, cross-wave vorticity $\left(\bar{\omega}_{y} /\left(k u_{* s}\right)\right)$, and along-wave vorticity $\left(-\bar{\omega}_{x} /\left(k u_{* s}\right)\right)$. 

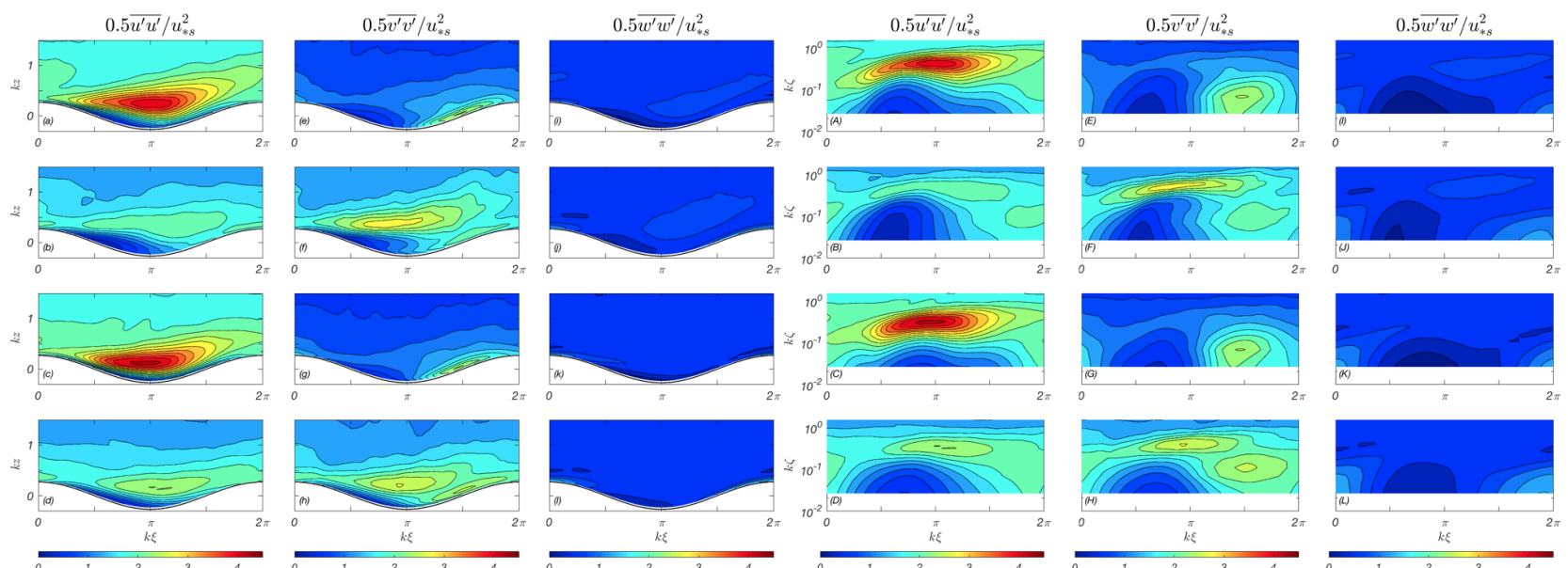

Figure 3.4: Normalized phase-averaged flow fields in the $\xi$ - $z$ coordinate (left three panels) and the mapped $\xi-\zeta$ coordinate (right three panels) for waves following wind (top two rows; oblique wind in second row) and for waves opposing wind (bottom two rows; oblique wind in fourth row). From left to right: the along-wave component $\left(0.5 \overline{u^{\prime} u^{\prime}} / u_{* s}^{2}\right)$, the cross-wave component $\left(0.5 \overline{v^{\prime} v^{\prime}} / u_{* s}^{2}\right)$, and the vertical component $\left(0.5 \overline{w^{\prime} w^{\prime}} / u_{* s}^{2}\right)$ of TKE. 

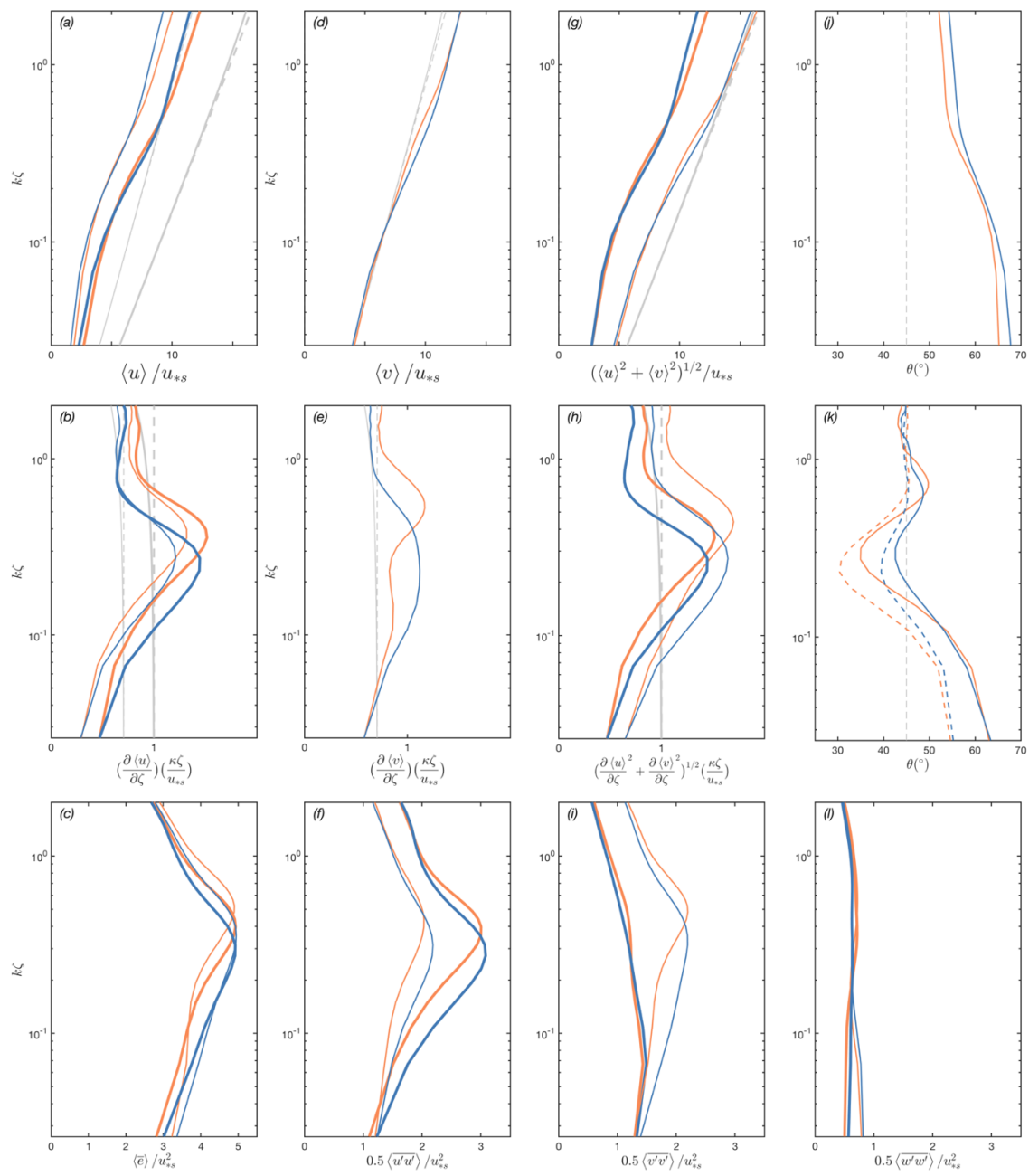

Figure 3.5: Top row from left to right: normalized vertical profiles of horizontally averaged along-wave wind speed $\left(\langle u\rangle / u_{* s}\right)$, cross-wave wind speed $\left(\langle v\rangle / u_{* s}\right)$, wind speed magnitude, and wind speed angle. Middle row from left to right: normalized vertical profiles of horizontally averaged along-wave wind shear $\left(\frac{\partial\langle u\rangle}{\partial \zeta} \frac{\kappa \zeta}{u_{* s}}\right)$, cross-wave wind shear $\left(\frac{\partial\langle v\rangle}{\partial \zeta} \frac{\kappa \zeta}{u_{* s}}\right)$, wind shear magnitude, and wind shear angle. In panel $(\mathrm{k})$ dashed lines show angle of horizontally averaged turbulent stress vector $\left(\left\langle\tau_{13}^{t}\right\rangle,\left\langle\tau_{23}^{t}\right\rangle\right)$. Bottom row from left to right: normalized vertical profiles of horizontally averaged TKE magnitude $\left(\langle\bar{e}\rangle / u_{* s}^{2}\right)$, the along-wave component $\left(0.5\left\langle\overline{u^{\prime} u^{\prime}}\right\rangle / u_{* s}^{2}\right)$, the crosswave component $\left(0.5\left\langle\overline{v^{\prime} v^{\prime}}\right\rangle / u_{* s}^{2}\right)$, and the vertical component $\left(0.5\left\langle\overline{w^{\prime} w^{\prime}}\right\rangle / u_{* s}^{2}\right)$ of the TKE. In all panels profiles for waves following (opposing) aligned wind are shown as thick orange (blue) lines, and profiles for waves following (opposing) oblique wind are shown as thin orange (blue) lines. Grey lines show profiles for flat wall cases (explained in the main text). 

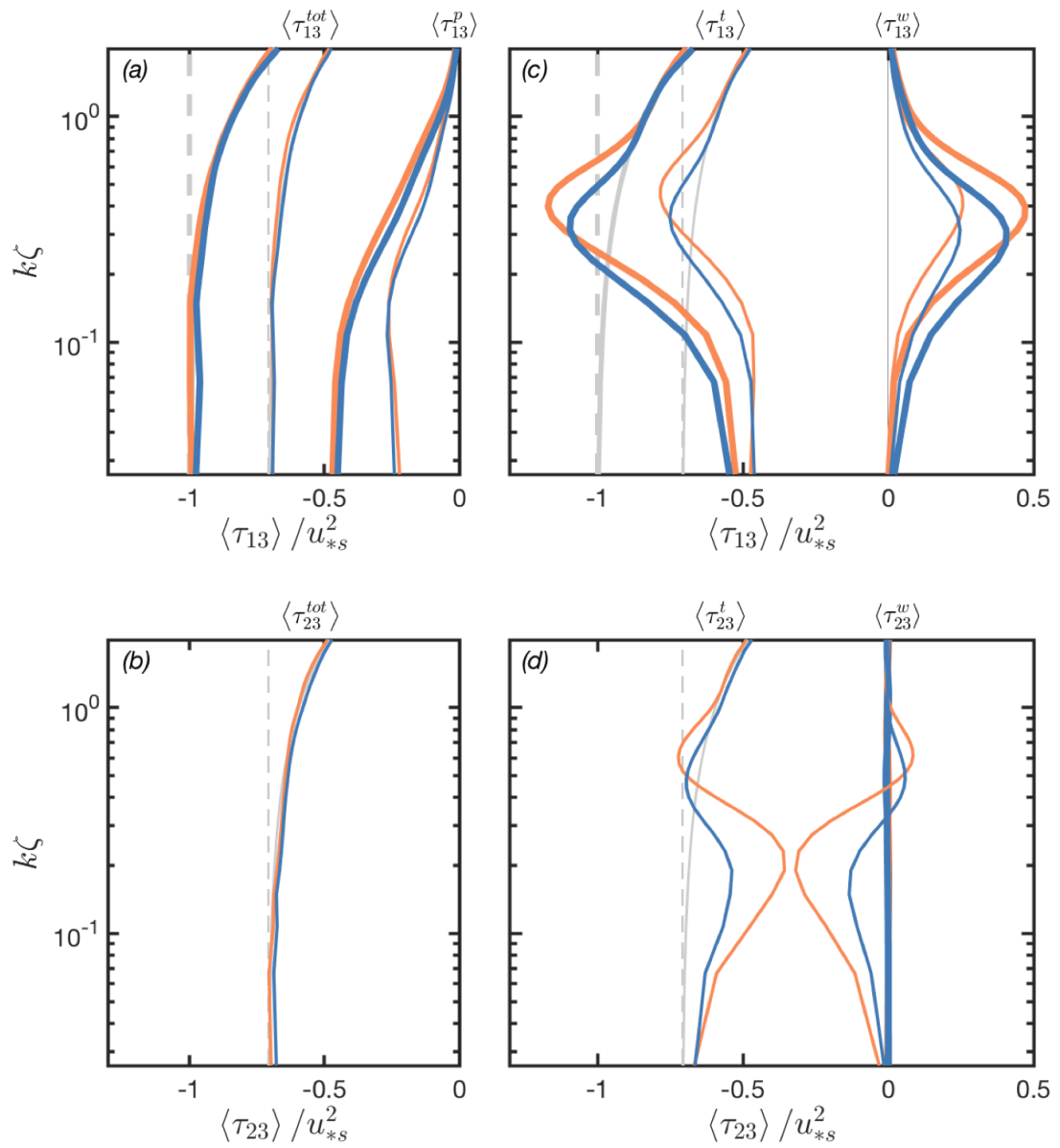

Figure 3.6: Normalized vertical profiles of horizontally averaged momentum budget terms following wind (orange lines) and opposing wind (blue lines) for oblique (thin lines) and aligned (thick lines) wind conditions. In the top row, the along-wave (x) momentum budget includes total wind stress in the $x$-direction $\left(\left\langle\tau_{13}^{t o t}\right\rangle / u_{* s}^{2}\right)$, pressure stress $\left(\left\langle\tau_{13}^{p}\right\rangle / u_{* s}^{2}\right)$, along-wave turbulent stress $\left(\left\langle\tau_{13}^{t}\right\rangle / u_{* s}^{2}\right)$, and along-wave wave-coherent stress $\left(\left\langle\tau_{13}^{w}\right\rangle / u_{* s}^{2}\right)$. In the bottom row, the cross-wave $(y)$ momentum budget includes total wind stress in the $y$-direction $\left(\left\langle\tau_{23}^{t o t}\right\rangle / u_{* s}^{2}\right)$, cross-wave turbulent stress $\left(\left\langle\tau_{23}^{t}\right\rangle / u_{* s}^{2}\right)$, and cross-wave wave-coherent stress $\left(\left\langle\tau_{23}^{w}\right\rangle / u_{* s}^{2}\right)$. Grey lines show profiles for flat wall cases (explained in the main text). 

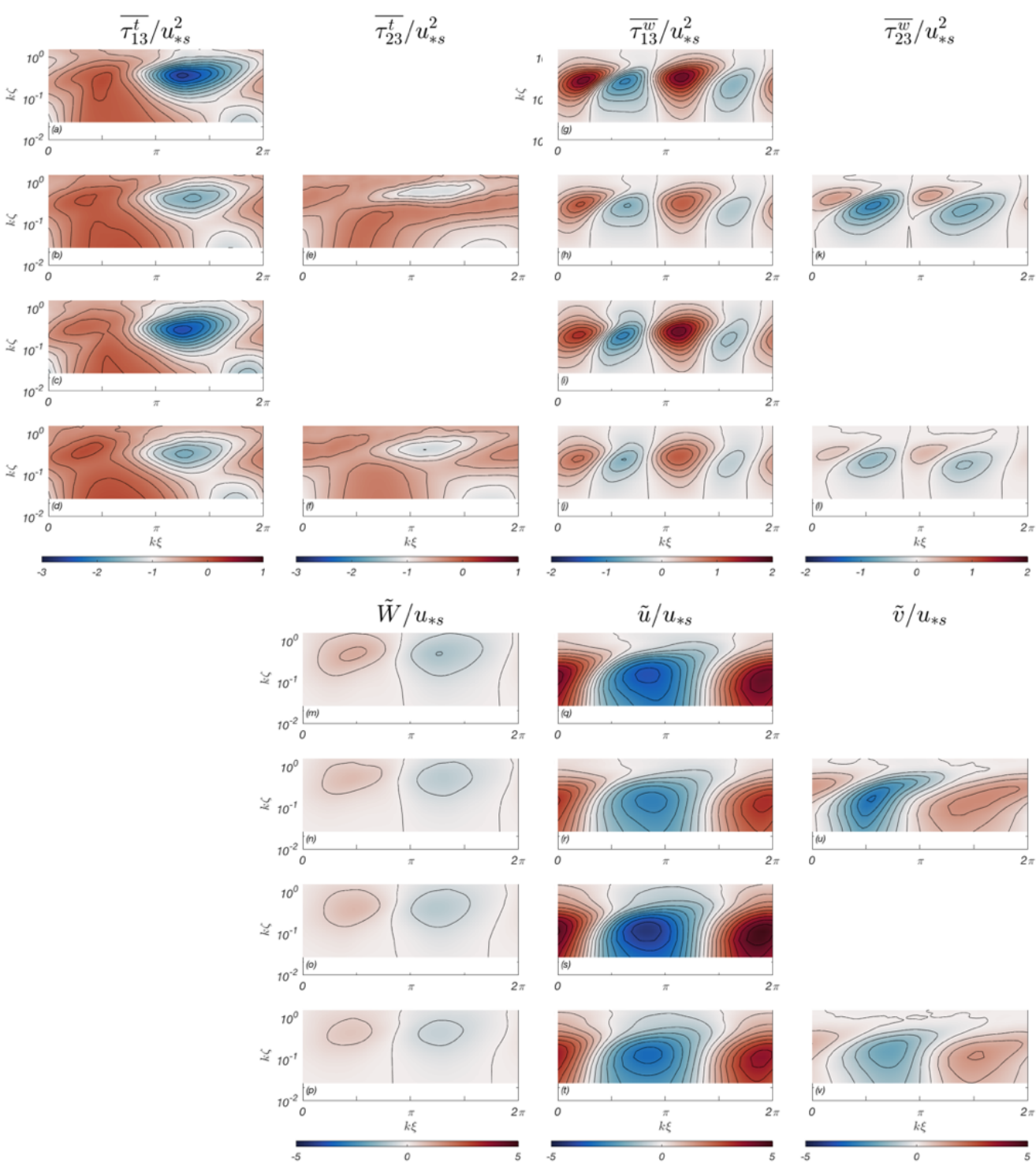

Figure 3.7: Top four panels: Normalized phase-averaged fields of the alongwave turbulent stress $\left(\overline{\tau_{13}^{t}} / u_{* s}^{2}=\overline{u^{\prime} W^{\prime}} / u_{* s}^{2}\right)$, cross-wave turbulent stress $\left(\overline{\tau_{23}^{t}} / u_{* s}^{2}=\right.$ $\left.\overline{v^{\prime} W^{\prime}} / u_{* s}^{2}\right)$, along-wave wave-coherent stress $\left(\overline{\tau_{13}^{w}} / u_{* s}^{2}=\tilde{u} \tilde{W} / u_{* s}^{2}\right)$, and cross-wave wave-coherent stress $\left(\overline{\tau_{23}^{w}} / u_{* s}^{2}=\tilde{v} \tilde{W} / u_{* s}^{2}\right)$ in the mapped $\xi$ - $\zeta$ coordinate. From top to bottom: waves following aligned wind, waves following oblique wind, wave opposing aligned wind, and waves opposing oblique wind. Bottom four panels: Normalized phase-averaged fields of the wave-coherent vertical velocity $\left(\tilde{W} / u_{* s}\right)$, along-wave velocity $\left(\tilde{u} / u_{* s}\right)$, and cross-wave velocity $\left(\tilde{v} / u_{* s}\right)$ in the mapped $\xi-\zeta$ coordinate. 

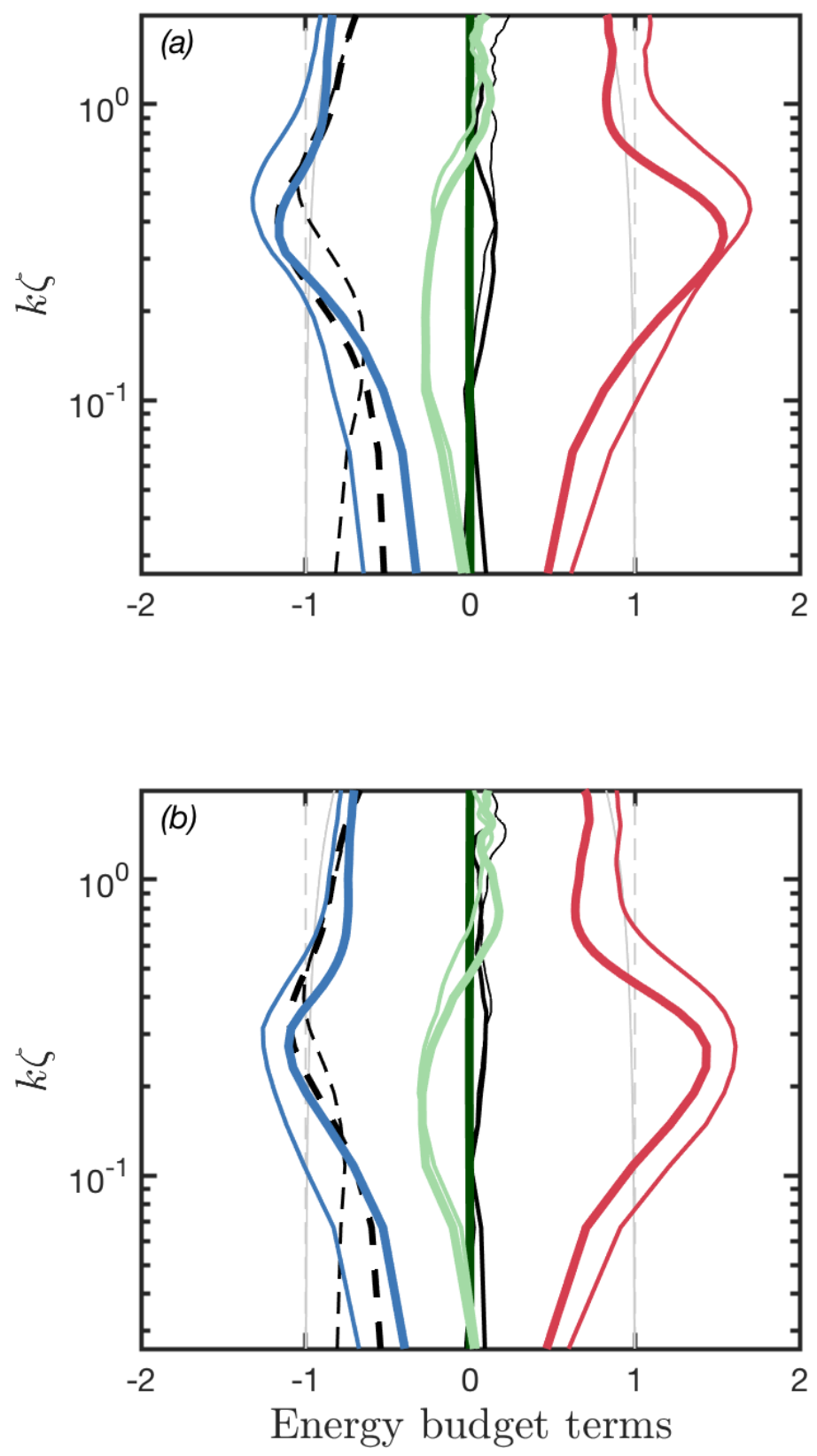

Figure 3.8: Normalized vertical profiles of horizontally averaged energy budget terms. The first, second, third and fourth terms of Equation 3.3 are dark green, dark red, light green, and blue lines, respectively. Thick (thin) lines are for aligned (oblique) wind. Solid black lines are sum of all energy budget terms. Top (bottom) panel is for waves following (opposing) wind. 


\section{References}

[1] Al-Zanaidi, M. A., and W. H. Hui, 1984: Turbulent airflow over water waves-a numerical study. Journal of Fluid Mechanics, 148, 225-246, doi: 10.1017/S0022112084002329, publisher: Cambridge University Press.

[2] Andreas, E. L., 2004: Spray Stress Revisited. Journal of Physical Oceanography, 34 (6), 1429-1440, doi:10.1175/1520-0485(2004)034〈1429:SSR〉2.0.CO;2, publisher: American Meteorological Society.

[3] Ardhuin, F., T. H. C. Herbers, K. P. Watts, G. P. van Vledder, R. Jensen, and H. C. Graber, 2007: Swell and Slanting-Fetch Effects on Wind Wave Growth. Journal of Physical Oceanography, 37 (4), 908-931, doi:10.1175/JPO3039.1, publisher: American Meteorological Society.

[4] Banner, M. L., 1990: The influence of wave breaking on the surface pressure distribution in wind-wave interactions. Journal of Fluid Mechanics, 211, 463-495.

[5] Banner, M. L., and W. K. Melville, 1976: On the separation of air flow over water waves. Journal of Fluid Mechanics, 77 (4), 825-842.

[6] Belcher, S. E., and J. C. R. Hunt, 1993: Turbulent shear flow over slowly 
moving waves. Journal of Fluid Mechanics, 251 (-1), 109, doi:10.1017/ S0022112093003350.

[7] Belcher, S. E., and J. C. R. Hunt, 1998: Turbulent flow over hills and waves. Annual Review of Fluid Mechanics, 30 (1), 507-538.

[8] Belcher, S. E., T. M. J. Newley, and J. C. R. Hunt, 1993: The drag on an undulating surface induced by the flow of a turbulent boundary layer. Journal of fluid mechanics, 249, 557-596.

[9] Black, P. G., and Coauthors, 2007: Air-Sea Exchange in Hurricanes: Synthesis of Observations from the Coupled Boundary Layer Air-Sea Transfer Experiment. Bulletin of the American Meteorological Society, 88 (3), 357-374, doi:10.1175/BAMS-88-3-357.

[10] Bourassa, M. A., D. G. Vincent, and W. L. Wood, 1999: A Flux Parameterization Including the Effects of Capillary Waves and Sea State. Journal of the Atmospheric Sciences, 56 (9), 1123-1139, doi:10.1175/1520-0469(1999) 056〈1123:AFPITE〉2.0.CO;2, publisher: American Meteorological Society.

[11] Buckley, M., 2015: Structure of the airflow above surface waves. Thesis, University of Delaware.

[12] Buckley, M. P., and F. Veron, 2016: Structure of the Airflow above Surface Waves. Journal of Physical Oceanography, 46 (5), 1377-1397, doi:10.1175/ JPO-D-15-0135.1.

[13] Buckley, M. P., and F. Veron, 2017: Airflow measurements at a wavy air-water interface using PIV and LIF. Experiments in Fluids, 58 (11), 161, doi: 10.1007/s00348-017-2439-2. 
[14] Buckley, M. P., and F. Veron, 2019: The turbulent airflow over wind generated surface waves. European Journal of Mechanics - B/Fluids, 73, 132-143, doi: 10.1016/j.euromechflu.2018.04.003.

[15] Burgers, G., and V. K. Makin, 1993: Boundary-Layer Model Results for Wind-Sea Growth. Journal of Physical Oceanography, 23 (2), 372-385, doi: 10.1175/1520-0485(1993)023〈0372:BLMRFW 〉2.0.CO;2, publisher: American Meteorological Society.

[16] Cao, T., B.-Q. Deng, and L. Shen, 2020: A simulation-based mechanistic study of turbulent wind blowing over opposing water waves. Journal of Fluid Mechanics, 901, doi:10.1017/jfm.2020.591, publisher: Cambridge University Press.

[17] Chalikov, D., and S. Rainchik, 2011: Coupled Numerical Modelling of Wind and Waves and the Theory of the Wave Boundary Layer. Boundary-Layer Meteorology, 138 (1), 1-41, doi:10.1007/s10546-010-9543-7.

[18] Chen, S. S., and M. Curcic, 2016: Ocean surface waves in Hurricane Ike (2008) and Superstorm Sandy (2012): Coupled model predictions and observations. Ocean Modelling, 103, 161-176, doi:10.1016/j.ocemod.2015.08.005.

[19] Chen, X., I. Ginis, and T. Hara, 2020: Impact of Shoaling Ocean Surface Waves on Wind Stress and Drag Coefficient in Coastal Waters: 2. Tropical Cyclones. Journal of Geophysical Research: Oceans, 125 (7), e2020JC016223, doi:10.1029/2020JC016223, _eprint: https://agupubs.onlinelibrary.wiley.com/doi/pdf/10.1029/2020JC016223. 
[20] Cohen, J. E., 1997: Theory of turbulent wind over fast and slow waves. Ph.D., University of Cambridge, doi:10.17863/CAM.31086, accepted: 1997.

[21] Cronin, M. F., and Coauthors, 2019: Air-Sea Fluxes With a Focus on Heat and Momentum. Frontiers in Marine Science, 6, doi:10.3389/fmars.2019.00430.

[22] Degani, A. T., J. D. A. Walker, and F. T. Smith, 1998: Unsteady separation past moving surfaces. Journal of Fluid Mechanics, 375, 1-38, doi:10.1017/ S0022112098001839, publisher: Cambridge University Press.

[23] Deike, L., L. Lenain, and W. K. Melville, 2017: Air entrainment by breaking waves. Geophysical Research Letters, 44 (8), 3779-3787, doi:10.1002/2017GL072883, _eprint: https://onlinelibrary.wiley.com/doi/pdf/10.1002/2017GL072883.

[24] Deike, L., and W. K. Melville, 2018: Gas Transfer by Breaking Waves. Geophysical Research Letters, 45 (19), 10,482-10,492, doi:10.1029/2018GL078758, _eprint: https://onlinelibrary.wiley.com/doi/pdf/10.1029/2018GL078758.

[25] Donelan, M., 1999: Wind-induced growth and attenuation of laboratory waves. Wind-over-Wave Couplings: Perspectives and Prospects, 69, sajjadi et al. ed., Clarendon, 183-194.

[26] Donelan, M. A., 2004: On the limiting aerodynamic roughness of the ocean in very strong winds. Geophysical Research Letters, 31 (18), doi:10.1029/ 2004GL019460.

[27] Donelan, M. A., A. V. Babanin, I. R. Young, and M. L. Banner, 2006: Wave-follower field measurements of the wind-input spectral function. Part II: 
Parameterization of the wind input. Journal of physical oceanography, 36 (8), $1672-1689$.

[28] Donelan, M. A., M. Curcic, S. S. Chen, and A. K. Magnusson, 2012: Modeling waves and wind stress. Journal of Geophysical Research: Oceans, 117 (C11), C00J23, doi:10.1029/2011JC007787.

[29] Donelan, M. A., and F. W. Dobson, 2001: The Influence of Swell on the Drag. Wind Stress over the Ocean, I. S. F. Jones, and Y. Toba, Eds., 1st ed., Cambridge University Press, 181-189, doi:10.1017/CBO9780511552076.009.

[30] Donelan, M. A., F. W. Dobson, S. D. Smith, and R. J. Anderson, 1993: On the Dependence of Sea Surface Roughness on Wave Development. Journal of Physical Oceanography, 23 (9), 2143-2149, doi:10.1175/1520-0485(1993) 023〈2143:OTDOSS $\rangle 2.0 . \mathrm{CO} ; 2$.

[31] Donelan, M. A., W. M. Drennan, and K. B. Katsaros, 1997: The AirâSea Momentum Flux in Conditions of Wind Sea and Swell. Journal of Physical Oceanography, 27 (10), 2087-2099, doi:10.1175/1520-0485(1997)027〈2087: TASMFI 2.0.CO;2, publisher: American Meteorological Society.

[32] Drennan, W. M., H. C. Graber, and M. A. Donelan, 1999: Evidence for the Effects of Swell and Unsteady Winds on Marine Wind Stress. Journal of Physical Oceanography, 29 (8), 1853-1864, doi:10.1175/1520-0485(1999) 029〈1853:EFTEOS〉2.0.CO;2, publisher: American Meteorological Society.

[33] Druzhinin, O. A., Y. I. Troitskaya, and S. S. Zilitinkevich, 2012: Direct numerical simulation of a turbulent wind over a wavy water surface. Journal of Geo- 
physical Research: Oceans, 117 (C11), doi:10.1029/2011JC007789, _eprint: https://agupubs.onlinelibrary.wiley.com/doi/pdf/10.1029/2011JC007789.

[34] Edson, J., and Coauthors, 2007: The Coupled Boundary Layers and AirâSea Transfer Experiment in Low Winds. Bulletin of the American Meteorological Society, 88 (3), 341-356, doi:10.1175/BAMS-88-3-341, publisher: American Meteorological Society.

[35] Edson, J. B., and C. W. Fairall, 1998: Similarity Relationships in the Marine Atmospheric Surface Layer for Terms in the TKE and Scalar Variance Budgets. Journal of the Atmospheric Sciences, 55 (13), 2311-2328, doi: 10.1175/1520-0469(1998)055〈2311:SRITMA〉2.0.CO;2, publisher: American Meteorological Society.

[36] Edson, J. B., and Coauthors, 2013: On the Exchange of Momentum over the Open Ocean. Journal of Physical Oceanography, 43 (8), 1589-1610, doi:10.1175/JPO-D-12-0173.1.

[37] Fairall, C., J. Kepert, and G. Holland, 1995: The effect of sea spray on surface energy transports over the ocean. The Global Atmosphere and Ocean System, 2, 121-142.

[38] Fairall, C. W., E. F. Bradley, J. E. Hare, A. A. Grachev, and J. B. Edson, 2003: Bulk Parameterization of Air-Sea Fluxes: Updates and Verification for the COARE Algorithm. Journal of Climate, 16 (4), 571-591, doi:10.1175/1520-0442(2003)016〈0571:BPOASF >2.0.CO;2, publisher: American Meteorological Society.

[39] Fan, Y., I. Ginis, T. Hara, C. W. Wright, and E. J. Walsh, 2009: Numerical 
Simulations and Observations of Surface Wave Fields under an Extreme Tropical Cyclone. Journal of Physical Oceanography, 39 (9), 2097-2116, doi:10.1175/2009JPO4224.1, publisher: American Meteorological Society.

[40] Gad-el Hak, M., and D. M. Bushnell, 1991: Separation Control: Review. Journal of Fluids Engineering, 113 (1), 5-30, doi:10.1115/1.2926497, publisher: American Society of Mechanical Engineers Digital Collection.

[41] Geernaert, G. L., 1988: Measurements of the angle between the wind vector and wind stress vector in the surface layer over the North Sea. Journal of Geophysical Research: Oceans, 93 (C7), doi:https://doi.org/10.1029/ JC093iC07p08215.

[42] Geernaert, G. L., F. Hansen, M. Courtney, and T. Herbers, 1993: Directional attributes of the ocean surface wind stress vector. Journal of Geophysical Research: Oceans, 98 (C9), 16 571-16 582, doi:https://doi.org/10.1029/ 93JC01439.

[43] Gent, P. R., 1977: A numerical model of the air flow above water waves. Part 2. Journal of Fluid Mechanics, 82 (02), 349, doi:10.1017/S0022112077000706.

[44] Gent, P. R., and P. A. Taylor, 1976: A numerical model of the air flow above water waves. Journal of Fluid Mechanics, 77 (01), 105, doi:10.1017/ S0022112076001158.

[45] Gong, W., P. A. Taylor, and A. Dörnbrack, 1996: Turbulent boundarylayer flow over fixed aerodynamically rough two-dimensional sinusoidal waves. Journal of Fluid Mechanics, 312, 1-37, doi:10.1017/S0022112096001905. 
[46] Grachev, A. A., C. W. Fairall, J. E. Hare, J. B. Edson, and S. D. Miller, 2003: Wind Stress Vector over Ocean Waves. Journal of Physical Oceanography, 33 (11), 2408-2429, doi:10.1175/1520-0485(2003)033〈2408:WSVOOW $\rangle 2.0$. $\mathrm{CO} ; 2$.

[47] Grare, L., W. L. Peirson, H. Branger, J. W. Walker, J.-P. Giovanangeli, and V. Makin, 2013: Growth and dissipation of wind-forced, deep-water waves. Journal of Fluid Mechanics, 722, 5-50, doi:10.1017/jfm.2013.88.

[48] Hao, X., and L. Shen, 2019: Wind-wave coupling study using LES of wind and phase-resolved simulation of nonlinear waves. Journal of Fluid Mechanics, 874, 391-425, doi:10.1017/jfm.2019.444, publisher: Cambridge University Press.

[49] Hara, T., and S. E. Belcher, 2002: Wind forcing in the equilibrium range of wind-wave spectra. Journal of Fluid Mechanics, 470, doi:10.1017/ S0022112002001945.

[50] Hara, T., and S. E. Belcher, 2004: Wind profile and drag coefficient over mature ocean surface wave spectra. Journal of physical oceanography, 34 (11), 2345-2358.

[51] Hara, T., and P. Sullivan, 2015: Wave Boundary Layer Turbulence over Surface Waves in a Strongly Forced Condition. Graduate School of Oceanography Faculty Publications, doi:10.1175/JPO-D-14-0116.1.

[52] Harris, J., I. Fultion, and R. Street, 1995: Decay of Waves in an Adverse Wind. Proceedings of the Sixth Asian Congress of Fluid Mechanics, Y. Chew, and C. Tso, Eds., Singapore, 224-227, Fluid mechanics, meeting Name: Asian 
Congress of Fluid Mechanics (ACFM) Pages: 224-227 Publication Title: Decay of Waves in an Adverse Wind OCLC: 833209562.

[53] Hasselmann, D., and J. Bösenberg, 1991: Field measurements of wave-induced pressure over wind-sea and swell. Journal of Fluid Mechanics, 230, 391-428, doi:10.1017/S0022112091000848, publisher: Cambridge University Press.

[54] Högström, U., E. Sahlée, A.-S. Smedman, A. Rutgersson, E. Nilsson, K. K. Kahma, and W. M. Drennan, 2015: Surface Stress over the Ocean in Swell-Dominated Conditions during Moderate Winds. Journal of the Atmospheric Sciences, 72 (12), 4777-4795, doi:10.1175/JAS-D-15-0139.1, publisher: American Meteorological Society.

[55] Holthuijsen, L. H., M. D. Powell, and J. D. Pietrzak, 2012: Wind and waves in extreme hurricanes. Journal of Geophysical Research: Oceans, 117 (C9), doi:10.1029/2012JC007983.

[56] Husain, N. T., T. Hara, M. P. Buckley, K. Yousefi, F. Veron, and P. P. Sullivan, 2019: Boundary Layer Turbulence over Surface Waves in a Strongly Forced Condition: LES and Observation. Journal of Physical Oceanography, 49 (8), 1997-2015, doi:10.1175/JPO-D-19-0070.1.

[57] Kudryavtsev, V. N., and V. K. Makin, 2001: The Impact Of Air-Flow Separation On The Drag Of The Sea Surface. Boundary-Layer Meteorology, 98 (1), 155-171, doi:10.1023/A:1018719917275.

[58] Kudryavtsev, V. N., and V. K. Makin, 2002: Coupled dynamics of short waves and the airflow over long surface waves. Journal of Geophysical Research: Oceans, 107 (C12), 3209, doi:10.1029/2001JC001251. 
[59] Kudryavtsev, V. N., and V. K. Makin, 2004: Impact of Swell on the Marine Atmospheric Boundary Layer. Journal of Physical Oceanography, 34 (4), 934-949, doi:10.1175/1520-0485(2004)034〈0934:IOSOTM〉2.0.CO;2, publisher: American Meteorological Society.

[60] Kudryavtsev, V. N., and V. K. Makin, 2011: Impact of Ocean Spray on the Dynamics of the Marine Atmospheric Boundary Layer. Boundary-Layer Meteorology, 140 (3), 383-410, doi:10.1007/s10546-011-9624-2.

[61] Kudryavtsev, V. N., V. K. Makin, and J. F. Meirink, 2001: Simplified Model Of The Air Flow Above Waves. Boundary-Layer Meteorology, 100 (1), 63-90, doi:10.1023/A:1018914113697.

[62] Kukulka, T., and T. Hara, 2008: The Effect of Breaking Waves on a Coupled Model of Wind and Ocean Surface Waves. Part II: Growing Seas. Journal of Physical Oceanography, 38 (10), 2164-2184, doi:10.1175/2008JPO3962.1.

[63] Kukulka, T., T. Hara, and S. E. Belcher, 2007: A Model of the Air-Sea Momentum Flux and Breaking-Wave Distribution for Strongly Forced Wind Waves. Journal of Physical Oceanography, 37 (7), 1811-1828, doi:10.1175/ JPO3084.1.

[64] Large, W. G., and S. Pond, 1981: Open Ocean Momentum Flux Measurements in Moderate to Strong Winds. Journal of Physical Oceanography, 11 (3), 324336, doi:10.1175/1520-0485(1981)011〈0324:OOMFMI〉2.0.CO;2, publisher: American Meteorological Society.

[65] Li, P. Y., D. Xu, and P. A. Taylor, 2000: Numerical modelling of turbulent airflow over water waves. Boundary-layer meteorology, 95 (3), 397-425. 
[66] Makin, V. K., and V. N. Kudryavtsev, 1999: Coupled sea surface-atmosphere model: 1. Wind over waves coupling. Journal of Geophysical Research: Oceans, 104 (C4), 7613-7623, doi:10.1029/1999JC900006.

[67] Makin, V. K., and V. N. Kudryavtsev, 2002: Impact of dominant waves on sea drag. Boundary-Layer Meteorology, 103 (1), 83-99.

[68] Mastenbroek, C., 1996: Wind-wave interaction. Ph.D. thesis, Delft Technical University.

[69] Meirink, J. F., V. K. Makin, and V. N. Kudryavtsev, 2003: Note on the Growth Rate of Water Waves Propagating at an Arbitrary Angle to the Wind. Boundary-Layer Meteorology, 106 (1), 171-183, doi:10.1023/A: 1020835211837 .

[70] Mitsuyasu, H., and Y. Yoshida, 2005: Air-Sea Interactions under the Existence of Opposing Swell. Journal of Oceanography, 61 (1), 141-154, doi:10.1007/ s10872-005-0027-1.

[71] Moeng, C.-H., 1984: A Large-Eddy-Simulation Model for the Study of Planetary Boundary-Layer Turbulence. Journal of the Atmospheric Sciences, 41 (13), 2052-2062, doi:10.1175/1520-0469(1984)041〈2052:ALESMF〉2.0.CO; 2.

[72] Moeng, C.-H., and P. Sullivan, 2015: Large eddy simulation. Encyclopedia of Atmospheric Sciences, 2nd ed., Academic Press.

[73] Moon, I.-J., I. Ginis, T. Hara, H. L. Tolman, C. W. Wright, and E. J. Walsh, 2003: Numerical Simulation of Sea Surface Directional Wave Spectra 
under Hurricane Wind Forcing. Journal of Physical Oceanography, 33 (8), 1680-1706, doi:10.1175/2410.1.

[74] Moon, I.-J., T. Hara, I. Ginis, S. E. Belcher, and H. L. Tolman, 2004: Effect of Surface Waves on Air-Sea Momentum Exchange. Part I: Effect of Mature and Growing Seas. Journal of the Atmospheric Sciences, 61 (19), 2321-2333, doi:10.1175/1520-0469(2004)061〈2321:EOSWOA $\rangle 2.0 . C O ; 2$.

[75] Moon, I.-J., J.-I. Kwon, J.-C. Lee, J.-S. Shim, S. K. Kang, I. S. Oh, and S. J. Kwon, 2009: Effect of the surface wind stress parameterization on the storm surge modeling. Ocean Modelling, 29 (2), 115-127, doi:10.1016/j.ocemod. 2009.03.006.

[76] Mueller, J. A., and F. Veron, 2009: Nonlinear Formulation of the Bulk Surface Stress over Breaking Waves: Feedback Mechanisms from Air-flow Separation. Boundary-Layer Meteorology, 130 (1), 117-134, doi:10.1007/ s10546-008-9334-6.

[77] Oppenheim, A. V., and R. W. Schafer, 2013: Discrete-Time Signal Processing. Pearson Education, Limited.

[78] Patton, E. G., P. P. Sullivan, B. Kosović, J. Dudhia, L. Mahrt, M. Žagar, and T. Marić, 2019: On the Influence of Swell Propagation Angle on Surface Drag. Journal of Applied Meteorology and Climatology, 58 (5), 1039-1059, doi:10.1175/JAMC-D-18-0211.1.

[79] Peirson, W. L., and A. W. Garcia, 2008: On the wind-induced growth of slow water waves of finite steepness. Journal of Fluid Mechanics, 608, 243-274, doi:10.1017/S002211200800205X, publisher: Cambridge University Press. 
[80] Peirson, W. L., A. W. Garcia, and S. E. Pells, 2003: Water wave attenuation due to opposing wind. Journal of Fluid Mechanics, 487, 345-365, doi:10. 1017/S0022112003004750.

[81] Plant, W. J., 1982: A relationship between wind stress and wave slope. Journal of Geophysical Research, 87 (C3), 1961, doi:10.1029/JC087iC03p01961.

[82] Powell, M. D., P. J. Vickery, and T. A. Reinhold, 2003: Reduced drag coefficient for high wind speeds in tropical cyclones. Nature, 422 (6929), 279-283, doi:10.1038/nature01481.

[83] Reichl, B. G., T. Hara, and I. Ginis, 2014: Sea state dependence of the wind stress over the ocean under hurricane winds: Sea State Dependence of the Wind Stress. Journal of Geophysical Research: Oceans, 119 (1), 30-51, doi:10.1002/2013JC009289.

[84] Reul, N., H. Branger, and J.-P. Giovanangeli, 2008: Air Flow Structure Over Short-gravity Breaking Water Waves. Boundary-Layer Meteorology, 126 (3), 477-505, doi:10.1007/s10546-007-9240-3.

[85] Richter, D. H., and P. P. Sullivan, 2013: Sea surface drag and the role of spray. Geophysical Research Letters, 40 (3), 656-660, doi:10.1002/grl.50163.

[86] Roekel, L. V., B. Fox-Kemper, P. P. Sullivan, P. E. Hamlington, and S. Haney, 2012: The form and orientation of Langmuir cells for misaligned winds and waves. doi:10.1029/2011jc007516.

[87] Savelyev, I. B., M. P. Buckley, and B. K. Haus, 2020: The Impact of Nonbreaking Waves on Wind-Driven Ocean Surface Turbulence. Journal of Geophysical Research: Oceans, 125 (1), e2019JC015 573, doi:10.1029/2019JC015573. 
[88] Snyder, R. L., F. W. Dobson, J. A. Elliott, and R. B. Long, 1981: Array measurements of atmospheric pressure fluctuations above surface gravity waves. Journal of Fluid Mechanics, 102, 1-59, doi:10.1017/S0022112081002528, publisher: Cambridge University Press.

[89] Sullivan, P. P., M. L. Banner, R. P. Morison, and W. L. Peirson, 2018: Impacts of wave age on turbulent flow and drag of steep waves. Procedia IUTAM, 26, 174-183, doi:10.1016/j.piutam.2018.03.017.

[90] Sullivan, P. P., M. L. Banner, R. P. Morison, and W. L. Peirson, 2018: Turbulent Flow over Steep Steady and Unsteady Waves under Strong Wind Forcing. Journal of Physical Oceanography, 48 (1), 3-27, doi:10.1175/JPO-D-17-0118. 1.

[91] Sullivan, P. P., and J. C. McWilliams, 2010: Dynamics of Winds and Currents Coupled to Surface Waves. Annual Review of Fluid Mechanics, 42 (1), 19-42, doi:10.1146/annurev-fluid-121108-145541.

[92] Sullivan, P. P., J. C. McWilliams, and C.-H. Moeng, 2000: Simulation of turbulent flow over idealized water waves. Journal of Fluid Mechanics, 404, $47-85$.

[93] Sullivan, P. P., J. C. McWilliams, and E. G. Patton, 2014: Large-Eddy Simulation of Marine Atmospheric Boundary Layers above a Spectrum of Moving Waves. Journal of the Atmospheric Sciences, 71 (11), 4001-4027, doi:10.1175/JAS-D-14-0095.1.

[94] Suzuki, N., T. Hara, and P. P. Sullivan, 2013: Impact of Breaking Wave Form Drag on Near-Surface Turbulence and Drag Coefficient over Young 
Seas at High Winds. Journal of Physical Oceanography, 43 (2), 324-343, doi:10.1175/JPO-D-12-0127.1.

[95] Suzuki, N., Y. Toba, and S. Komori, 2010: Examination of drag coefficient with special reference to the windsea Reynolds number: Conditions with counter and mixed swell. Journal of Oceanography, 66 (5), 731-739, doi: 10.1007/s10872-010-0060-6.

[96] Taylor, P. K., and M. J. Yelland, 2001: The Dependence of Sea Surface Roughness on the Height and Steepness of the Waves. Journal of Physical Oceanography, 31 (2), 572-590, doi:10.1175/1520-0485(2001)031<0572: TDOSSR $>2.0 . \mathrm{CO} ; 2$.

[97] Teixeira, M. A. C., 2018: A Model for the Wind-Driven Current in the Wavy Oceanic Surface Layer: Apparent Friction Velocity Reduction and Roughness Length Enhancement. Journal of Physical Oceanography, 48 (11), 2721-2736, doi:10.1175/JPO-D-18-0086.1.

[98] Tolman, H. L., and D. Chalikov, 1996: Source Terms in a Third-Generation Wind Wave Model. Journal of Physical Oceanography, 26 (11), 2497-2518, doi:10.1175/1520-0485(1996)026<2497:STIATG〉2.0.CO;2, publisher: American Meteorological Society.

[99] Troitskaya, Y., D. Sergeev, O. Ermakova, and G. Balandina, 2011: Statistical Parameters of the Air Turbulent Boundary Layer over Steep Water Waves Measured by the PIV Technique. Journal of Physical Oceanography, 41 (8), 1421-1454, doi:10.1175/2011JPO4392.1, publisher: American Meteorological Society. 
[100] Troitskaya, Y., D. A. Sergeev, O. Druzhinin, A. A. Kandaurov, O. S. Ermakova, E. V. Ezhova, I. Esau, and S. Zilitinkevich, 2014: Atmospheric boundary layer over steep surface waves. Ocean Dynamics, 64 (8), 11531161, doi:10.1007/s10236-014-0743-4.

[101] Uz, B. M., M. A. Donelan, T. Hara, and E. J. Bock, 2002: Laboratory Studies Of Wind Stress Over Surface Waves. Boundary-Layer Meteorology, 102 (2), 301-331, doi:10.1023/A:1013119313063.

[102] Veron, F., 2015: Ocean Spray. Annual Review of Fluid Mechanics, 47 (1), 507-538, doi:10.1146/annurev-fluid-010814-014651.

[103] Veron, F., G. Saxena, and S. K. Misra, 2007: Measurements of the viscous tangential stress in the airflow above wind waves. Geophysical Research Letters, 34 (19), doi:10.1029/2007GL031242.

[104] Walsh, E. J., and Coauthors, 2002: Hurricane Directional Wave Spectrum Spatial Variation at Landfall. Journal of Physical Oceanography, 32 (6), 16671684, doi:10.1175/1520-0485(2002)032〈1667:HDWSSV $>2.0 . C O ; 2$, publisher: American Meteorological Society.

[105] Wang, D., T. Kukulka, B. G. Reichl, T. Hara, and I. Ginis, 2019: Wind-Wave Misalignment Effects on Langmuir Turbulence in Tropical Cyclone Conditions. Journal of Physical Oceanography, 49 (12), 3109-3126, doi:10.1175/JPO-D-19-0093.1, publisher: American Meteorological Society.

[106] Wright, C. W., and Coauthors, 2001: Hurricane Directional Wave Spectrum Spatial Variation in the Open Ocean. JOURNAL OF PHYSICAL OCEANOGRAPHY, 31, 17. 
[107] Yang, D., and L. Shen, 2010: Direct-simulation-based study of turbulent flow over various waving boundaries. Journal of Fluid Mechanics, 650, 131, doi:10.1017/S0022112009993557.

[108] Yang, D., and L. Shen, 2017: Direct numerical simulation of scalar transport in turbulent flows over progressive surface waves. Journal of Fluid Mechanics, 819, 58-103, doi:10.1017/jfm.2017.164.

[109] Yang, Z., B.-Q. Deng, and L. Shen, 2018: Direct numerical simulation of wind turbulence over breaking waves. Journal of Fluid Mechanics, 850, 120-155, doi:10.1017/jfm.2018.466.

[110] Young, I. R., and R. J. Sobey, 1985: Measurements of the wind-wave energy flux in an opposing wind. Journal of Fluid Mechanics, 151, 427-442, doi: 10.1017/S0022112085001033, publisher: Cambridge University Press.

[111] Zavadsky, A., and L. Shemer, 2012: Characterization of turbulent airflow over evolving water-waves in a wind-wave tank. Journal of Geophysical Research: Oceans, 117 (C11), doi:10.1029/2011JC007790. 